\title{
RANDOM MATRICES CLOSE TO HERMITIAN OR UNITARY: overview of methods and results
}

\author{
Yan V. Fyodorov ${ }^{\S}$ and H.-J.Sommers $\llbracket$ \\ $\S$ Department of Mathematical Sciences, Brunel University, Uxbridge UB83PH, \\ United Kingdom \\ ๆ Fachbereich Physik, Universität Essen, D-45117 Essen, Germany
}

\begin{abstract}
The paper discusses recent progress in understanding statistical properties of eigenvalues of (weakly) non-Hermitian and non-unitary random matrices. The first type of ensembles is of the form $\hat{J}=\hat{H}-i \hat{\Gamma}$, with $\hat{H}$ being a large random $N \times N$ Hermitian matrix with independent entries "deformed" by a certain anti-Hermitian $N \times N$ matrix $i \hat{\Gamma}$ satisfying in the limit of large dimension $N$ the condition: $\operatorname{Tr} \hat{H}^{2} \propto N \operatorname{Tr} \hat{\Gamma}^{2}$. Here $\hat{\Gamma}$ can be either a random or just a fixed given Hermitian matrix. Ensembles of such a type with $\hat{\Gamma} \geq 0$ emerge naturally when describing quantum scattering in systems with chaotic dynamics and serve to describe resonance statistics. Related models are used to mimic complex spectra of the Dirac operator with chemical potential in the context of Quantum Chromodynamics.

Ensembles of the second type, arising naturally in scattering theory of discrete-time systems, are formed by $N \times N$ matrices $\hat{A}$ with complex entries such that $\hat{A}^{\dagger} \hat{A}=\hat{I}-\hat{T}$. For $\hat{T}=0$ this coincides with the Circular Unitary Ensemble, and $0 \leq \hat{T} \leq \hat{I}$ describes deviation from unitarity. Our result amounts to answering statistically the following old question: given the singular values of a matrix $\hat{A}$ describe the locus of its eigenvalues.

We systematically show that the obtained expressions for the correlation functions of complex eigenvalues describe a non-trivial crossover from WignerDyson statistics of real/unimodular eigenvalues typical for Hermitian/unitary matrices to Ginibre statistics in the complex plane typical for ensembles with strong non-Hermiticity: $<\operatorname{Tr} \hat{H}^{2}>\propto<\operatorname{Tr} \hat{\Gamma}^{2}>$ when $N \rightarrow \infty$. Finally we discuss (scarce) results available on eigenvector statistics for weakly nonHermitian random matrices.
\end{abstract}

\section{Introduction}

As is well-known, the statistics of highly excited bound states of closed quantum chaotic systems of quite different microscopic nature is universal. Namely, it turns out to be independent of the microscopic details when sampled on energy intervals large in comparison with the mean level spacing $\Delta$, but smaller than the so called Thouless energy scale. The latter is related by the Heisenberg uncertainty principle to the relaxation time necessary for the classically chaotic system to reach equilibrium in phase space [1]. Moreover, the spectral correlation functions turn out to be exactly those which are provided by the theory of large random Hermitian matrices with independent, identically distributed Gaussian entries. The correspondence holds in the limit of large matrix dimension on the so-called local scale. The 
local scale is determined by the typical separation $\Delta=\left\langle X_{i}-X_{i-1}\right\rangle$ between neighbouring eigenvalues situated around a point $X$, with the brackets standing for the statistical averaging. Microscopic arguments supporting the use of random matrices for describing the universal properties of quantum chaotic systems have been provided recently by several groups, based both on traditional semiclassical periodic orbit expansions [2, 3] and on advanced field-theoretical methods [4, 5].

In parallel, it was realized that another type of random matrices - those with the so-called chiral structure - serve as a pattern for universal statistics of low-lying eigenvalues of the Euclidean Dirac operator. The latter is of crucial importance for describing such a fundamental phenomenon as spontaneous breakdown of chiral symmetry in Quantum Chromodynamics (QCD). That line of development is thoroughly described in [6]. The corresponding (anti-)Hermitian matrices are of the form

$$
\hat{D}=i\left(\begin{array}{cc}
\mathbf{0} & \hat{J} \\
\hat{J}^{\dagger} & \mathbf{0}
\end{array}\right)
$$

where $\hat{J}$ stands for a complex matrix, with $\hat{J}^{\dagger}$ being its Hermitian conjugate. Such an off-diagonal block structure is characteristic for systems with chiral symmetry. One of the main objects of interest in QCD is the so-called partition function used to describe a system of quarks characterized by $N_{F}$ flavours and quark masses $m_{f}$ interacting with the Yang-Mills gauge fields. At the level of Random Matrix Theory the true partition function is replaced by the matrix integral:

$$
\mathcal{Z}_{N_{F}}(m)=\int d \hat{J} d \hat{J}^{\dagger} \prod_{f=1}^{N_{F}} \operatorname{det}\left\{\hat{D}+m_{f}\right\} e^{-N \operatorname{Tr} \hat{J}^{\dagger} \hat{J}}
$$

Here instead of an integration over gauge fields in the QCD partition function we have the integration over matrices $\hat{J}[\underline{6}$. It represents, in fact, the ensemble average of the product of characteristic polynomials of $\hat{D}$.

The Hermitian ensemble of block off-diagonal matrices $-i \hat{D}$ with independent, identically distributed Gaussian entries is known as the chiral Gaussian unitary ensemble (chGUE). The eigenvalues of chiral matrices appear in pairs $\pm \lambda_{k}, k=$ $1, \ldots, N$. It is evident that the nonzero $\epsilon_{k}=\lambda_{k}^{2} \neq 0$ are the singular values of the matrix $\hat{J}$, i.e. the eigenvalues of $\hat{J}^{\dagger} \hat{J}$. The latter are described by the so-called Laguerre ensemble which independently emerged in the description of quantum transport in mesoscopic systems [7], see e.g. [8] and references therein.

All these facts make the theory of random Hermitian matrices of various kinds a powerful and versatile tool of research in different branches of modern theoretical physics [1, 8.

Very recently complex eigenvalues of non-Hermitian random matrices have also attracted much research interest due to their relevance to several branches of theoretical physics. Most obvious motivation comes from the quantum description of open (or scattering) quantum chaotic systems 9, 10, 11, 12] whose fragments can escape, at a given energy, to infinity or come from infinity. For systems of this kind the notion of discrete energy levels loses its validity. Actually, chaotic scattering manifests itself in terms of a high density of poles of the scattering matrix placed irregularly in the complex energy plane. Each of these poles, or resonances, $\mathcal{E}_{k}=E_{k}-\frac{i}{2} \Gamma_{k}$, is characterized not only by energy $E_{k}$ but also by a finite width $\Gamma_{k}$ defined as (twice) the imaginary part of the corresponding complex energy and reflecting the finite lifetime 
of the states in the open system. Naturally, such a picture stimulated various groups to develop a statistical description of resonance parameters [13, 14, 15, 16, 17.

Recently, the progress in numerical techniques and computational facilities made available high accuracy patterns of resonance poles for realistic atomic and molecular systems in the regime of quantum chaos, see e.g. [18, 19, 20, 21] as well as for such model quantum chaotic systems as quantum graphs [22] and a quantum particle under time- and space-periodic perturbation [23. Due to the presence of resonances the elements of the scattering matrix show irregular fluctuations as functions of the energy of incoming waves, see 24] and references therein. The main goal of the theory of quantum chaotic scattering is to provide an adequate statistical description of such a behaviour.

The most natural framework for incorporating a random matrix description of the chaotic scattering (and hence for addressing statistics of resonances) is the so-called "Heidelberg approach" suggested in the pioneering paper [9] and described in much detail in [11, 12. The starting point of that approach is a particular formulation of the scattering theory based on the notion of an "effective Hamiltonian". Such formulation goes back to the ideas introduced in the context of Nuclear Physics in classical works by Kapur and Peierls, Wigner, Bloch, Feshbach and others. It was further developed, refined and clearly presented in the book by Mahaux and Weidenmüller [25], see also recent discussions in [12, 26]. Following that approach one introduces a division of the Hilbert space of the scattering system into two parts: the "interaction region" and the "channel region". The channel region is supposed to describe a situation of two fragments being apart far enough to neglect any interaction between them. Under these conditions their motion along the collision coordinate is described by a superposition of incoming and outgoing plane waves with wavevectors depending on the internal quantum states of the fragments. We assume that at given energy $E$ exactly $M$ different quantum states of the fragments are allowed, defining $M$ "scattering channels" numbered by the index $a$. At the same time, the second part of the Hilbert space is to describe the situation when fragments are close to one another and interact strongly. Correspondingly, any wavefunction of the system can be represented as a two-component vector, with first (second) component describing the wave function inside the interaction (channel) region.

Using the standard methods of scattering theory exposed in detail in [11, 12, 26] one can relate the two parts of the wave function to one another and finally arrive at the following representation of the energy-dependent scattering matrix $\hat{S}$ in terms of an effective non-Hermitian Hamiltonian $\mathcal{H}_{\text {eff }}=\hat{H}-i \hat{\Gamma}$ :

$$
S_{a b}(E)=\delta_{a b}-2 i \pi \sum_{i j} W_{a i}^{*}\left[E-\mathcal{H}_{e f f}\right]_{i j}^{-1} W_{j b}
$$

with the Hermitian Hamiltonian $\hat{H}$ describing the closed counterpart of the open system (i.e. interaction region decoupled from the channel one) and the anti-Hermitian part $i \hat{\Gamma}$ arising due to a coupling to open scattering channels. In this expression the Hamiltonian $\hat{H}$ is written in some arbitrary basis of states $|i\rangle$, such that $H_{i j}=\langle i|\hat{H}| j\rangle$. The amplitudes $W_{a i}, \quad a=1,2, \ldots, M$ are matrix elements coupling the internal motion in an "internal" state $|i\rangle$ to one out of M open channels $a$. One also has to choose the anti-Hermitian part to be $\Gamma_{i j}=\pi \sum_{a} W_{i a} W_{j a}^{*}$ in order to ensure the unitarity of the $M \times M$ scattering matrix $\hat{S}(E)$ [25]. The eigenvalues $\mathcal{E}_{k}$ of the operator $\mathcal{H}_{e f f}=\hat{H}-i \hat{\Gamma}$ are poles of the scattering matrix and have a physical interpretation as resonances: long-lived intermediate states to which discrete energy levels of the 
closed system are transformed due to coupling to continua. In particular, one can show that 11]

$$
\operatorname{det} S(E)=\frac{\operatorname{det}\left(E-\mathcal{H}_{\text {eff }}^{\dagger}\right)}{\operatorname{det}\left(E-\mathcal{H}_{\text {eff }}\right)}=\prod_{k=1}^{N} \frac{\left(E-\mathcal{E}_{k}^{*}\right)}{\left(E-\mathcal{E}_{k}\right)}
$$

expressing the total scattering phaseshift $\phi(E)=\log \operatorname{det} S(E)$ in terms of the egenvalues $\mathcal{E}_{k}$.

Applying these constructions to the quantum chaotic scattering it is natural to expect that universal properties of scattering systems are inherited from the corresponding universality of the energy levels of their closed counterparts. Thus, we expect the scattering characteristics (in particular, the statistics of resonances and phaseshifts) could be adequately described by a random matrix approximation on a scale comparable with the typical level spacing $\Delta$. In contrast, a smooth energy dependence of $S$-matrix elements on a much larger energy scale must be systemspecific.

According to the general idea one incorporates the random matrix description of quantum chaotic systems by replacing the Hamiltonian $\hat{H}$ by a random matrix of appropriate symmetry. Namely, chaotic systems with preserved time-reversal invariance (TRI) should be described by matrices $H_{i j}$ which are real symmetric. On the other hand systems with broken TRI are modelled by complex Hermitian matrices from the Gaussian Unitary Ensemble (GUE) [1. The last step of the Heidelberg approach is performing the ensemble averaging non-perturbatively. Usually it is done in the framework of the so-called supersymmetry technique introduced initially by Efetov [29] and adopted to the description of quantum chaotic scattering by Verbaarschot, Weidenmüller and Zirnbauer [9].

The Heidelberg approach turns out to be a very powerful tool for extracting different universal properties of open chaotic systems, and many important quantities characterizing the chaotic scattering were successfully investigated along these lines 10, 11, 12, 30. In particular, analytical predictions of the distribution of resonance widths [11, 15] were found to be in good agreement with available data obtained numerically for realistic models of chaotic quantum scattering [22, 23, 31].

In fact, the outlined description of the wave scattering can be looked at as an integral part of the general theory of linear dynamic open systems in terms of the input-output approach. These ideas and relations were developed in system theory and engineering mathematics many years ago, see e.g. 27. going back to the pioneering works by M. Livšic [28]. Livšic himself stressed equivalence of his mathematical constructions to those used by nuclear physicists, but that theory and subsequent developments went practically unnoticed by the quantum-chaos community. A brief description of the main constructions and interpretations of the linear open systems approach (in particular, a short derivation of the equation Eq.(3)) can be found in 63] and is not repeated here. Let us only mention that the unitary scattering matrix $\hat{S}(E)$ is known in the mathematical literature as the characteristic matrix-function of the non-Hermitian fundamental operator $\mathcal{H}_{\text {eff }}$.

In the scattering theory leading to Eq. (3) the time was considered to be a continuous parameter. On the other hand, a very useful instrument in the analysis of classical Hamiltonian systems with chaotic dynamics are the so-called area-preserving chaotic maps [32, 33]. They appear naturally either as a mapping of the Poincaré section onto itself, or as result of a stroboscopic description of Hamiltonians which are 
periodic functions of time. Their quantum mechanical analogues are unitary operators which act on Hilbert spaces of finite large dimension $N$, and are often referred to as evolution, scattering or Floquet operators, depending on the given physical context. Their eigenvalues consist of $N$ points on the unit circle (eigenphases). Numerical studies of various classically chaotic systems suggest that the eigenphases conform statistically quite accurately the results obtained for unitary random matrices of a particular symmetry (Dyson circular ensembles).

Let us now imagine that a system represented by a chaotic map ("inner world") is embedded in a larger physical system ("outer world") in such a way that it describes particles which can come inside the region of chaotic motion and leave it after some time via $M$ open channels. Models of such type appeared, for example, in 34.

The general linear system theory [27] considers dynamical systems with discrete time as frequently as those with continuous time. The corresponding construction is discussed in 63] and we mention here only its gross features. For a closed linear system characterized by a wavefunction $\Psi$ the "stroboscopic" dynamics amounts to a linear unitary map $\Psi(n) \rightarrow \Psi(n+1)$, such that $\Psi(n+1)=\hat{u} \Psi(n)$. The unitary evolution operator $\hat{u}$ describes the "closed" inner state domain decoupled both from input and output spaces. Then a coupling that makes the system open must convert the evolution operator $u$ to a contractive operator $\hat{A}$ such that $\mathbf{1}-\hat{A}^{\dagger} \hat{A} \geq 0$. It is easy to understand that one can always choose $\hat{A}=\hat{u} \sqrt{\mathbf{1 - \hat { \tau }} \hat{\tau}^{\dagger}}$ where the matrix $\hat{\tau}$ is a rectangular $N \times M$ diagonal with the entries $\tau_{i j}=\delta_{i j} \tau_{j}, 1 \leq i \leq N, 1 \leq j \leq M$ (for $M \leq$ $N), \quad 0 \leq \tau_{j} \leq 1$. Such a form automatically ensures $\mathbf{1}-\hat{A}^{\dagger} \hat{A}=\tau \tau^{\dagger} \geq 0$ and suggests a clear interpretation of the constituents of the model. Namely, the equation $\Psi(n+1)=\hat{A} \Psi(n)$ describes an irreversible decay of any initial state $\Psi(0) \neq 0$ when an input signal is absent. On the other hand, assuming a nonzero input and zero initial state $\Psi(0)=0$ one can relate the (discrete) Fourier-transforms of the input and output signals at a frequency $\omega$ to each other by a $M \times M$ unitary matrix $\hat{S}(\omega)$ given by

$$
\hat{S}(\omega)=\sqrt{\mathbf{1 - \hat { \tau } ^ { \dagger }} \hat{\tau}}-\hat{\tau}^{\dagger} \frac{1}{e^{-i \omega} \mathbf{1}-\hat{A}} \hat{u} \hat{\tau} .
$$

This equation is a complete discrete-time analogue of Eq.(3). In particular, using the idenity $\operatorname{det}\left(1-\hat{A}_{1} \hat{A}_{2}\right)=\operatorname{det}\left(1-\hat{A}_{2} \hat{A}_{1}\right)$ valid for two arbitrary matrices $\hat{A}_{1,2}$ as well as the relation $\frac{1}{\sqrt{1-\hat{\tau}^{\dagger} \hat{\tau}}} \tau^{\dagger}=\tau^{\dagger} \frac{1}{\sqrt{1-\hat{\tau}^{\dagger}}}$ it is straightforward to verify that

$$
\operatorname{det} \hat{S}(\omega)=e^{-i \omega N} \frac{\operatorname{det}\left(\hat{A}^{\dagger}-e^{i \omega}\right)}{\operatorname{det}\left(e^{-i \omega}-\hat{A}\right)}=e^{-i \omega N} \prod_{k=1}^{N} \frac{\left(z_{k}^{*}-e^{i \omega}\right)}{\left(e^{-i \omega}-z_{k}\right)},
$$

where $z_{k}$ stand for the complex eigenvalues of the matrix $\hat{A}$ which all belong to the interior of the unit circle: $|z|<1$. This relation is an obvious analogue of Eq.(4) and gives another indication that $z_{n}$ indeed play the role of resonances for the discrete time systems.

Now assume that the motion outside the inner region is regular. According to the general strategy one should be able to describe generic features of quantized maps representing chaotic inner dynamics by choosing the matrix $\hat{u}$ to be a member of the Dyson circular ensemble of random matrices. Then one finds: $\hat{\tau}^{\dagger} \hat{\tau}=\mathbf{1}-|\overline{\hat{S}(\omega)}|^{2}$, with the bar standing for the averaging of $\hat{S}(\omega)$ in Eq. (51) over $\hat{u}$. Comparing this result with [10, 15] we see that the $M$ eigenvalues $0 \leq T_{a} \leq 1$ of the $M \times M$ matrix $\hat{\tau}^{\dagger} \hat{\tau}$ 
play the role of the so-called transmission coefficients and describe a particular way the chaotic region is coupled to the outer world.

This line of reasoning was motivated by recent papers reviewed in 23. The authors considered the Floquet description of a Bloch particle in a constant force and periodic driving. After some approximations the evolution of the system is described by a mapping: $\mathbf{c}_{n+1}=\mathbf{F} \mathbf{c}_{n}$, where the unitary Floquet operator $\mathbf{F}=\hat{S} \hat{U}$ is the product of a unitary "M-shift" $\hat{S}: S_{k l}=\delta_{l, k-M}, l, k=-\infty, \ldots, \infty$ and a unitary matrix $\hat{U}$. The latter is effectively of the form $\hat{U}=\operatorname{diag}\left(\hat{d}_{1}, \hat{u}, d_{2}\right)$, where $\hat{d}_{1,2}$ are (semi)infinite diagonal matrices and $\hat{u}$ can be taken from the ensemble of random $N \times N$ unitary matrices. One can check that such a dynamics can easily be brought to the standard form discussed above, with all $M$ diagonal elements of the $N \times M$ matrix $\tau$ are equal to unitył.

A direct inspection immediately shows that the non-vanishing eigenvalues of the fundamental operator $\hat{A}$ for the latter example coincide with those of a $(N-M) \times$ $(N-M)$ subblock of the random unitary matrix $\hat{u}$. Complex eigenvalues of such "truncations" of random unitary matrices were studied analytically by Życzkowski and Sommers in [36], and we reproduce their study in Sec.3.1.

In fact, truncated unitary matrices represent only a particular case of random contractions $\hat{A}$. The ensemble of general $N \times N$ random contractions $\hat{A}=\hat{u} \sqrt{\mathbf{1 - \hat { \tau }} \hat{\tau}^{\dagger}}$ describing a chaotic map with broken time-reversal symmetry can be shown to have the following probability measure in the matrix space:

$$
\mathcal{P}(\hat{A}) d \hat{A} \propto \delta\left(\hat{A}^{\dagger} \hat{A}-\hat{G}\right) d \hat{A} \quad, \quad \hat{G} \equiv \mathbf{1}-\hat{\tau} \hat{\tau}^{\dagger}
$$

where $d \hat{A}=\prod_{i j} d \operatorname{Re} A_{i j} d \operatorname{Im} A_{i j}$ and the normalization constant is independent of $\hat{G}$. It follows by averaging the matrix $\delta$-function $\delta\left(\hat{A}-\hat{u} \sqrt{\mathbf{1 - \hat { \tau } \hat { \tau } ^ { \dagger }}}\right)$ over Dyson's circular unitary ensemble. The $N \times N$ matrix $\hat{\tau} \hat{\tau}^{\dagger}=\mathbf{1}-\hat{G} \geq 0$ is natural to call the deviation matrix and we denote it $\hat{T}$. It has $M$ nonzero eigenvalues coinciding with the transmission coefficients $T_{a}$ introduced above. The particular choice $T_{i(\leq M)}=1, T_{i(>M)}=0$ corresponds to the case considered in [36]. Eq. (7]) describes an ensemble of subunitary matrices $\hat{A}$ with given eigenvalues of $\hat{A}^{\dagger} \hat{A}$, i.e. with specified singular values of the matrix $\hat{A}$. The question of characterizing the locus of complex eigenvalues for a matrix with prescribed singular values was considered in classical papers by Horn and Weyl [37]. In essence, we provide a statistical answer to this question. Indeed, rotating the matrices $\hat{A}$ by a general unitary transformation and averaging over the unitary group yields a natural measure on matrices $\hat{A}$ with given singular values. In section $\mathbf{3}$ we will derive the corresponding joint density of eigenvalues and their correlation functions.

Although the initial interest in non-Hermitian and non-unitary deformations of random matrices had its origin in the theory of quantum chaotic scattering, soon after it got a much of stimulus from the QCD applications of chiral random matrices. The reason is that in order to describe Dirac eigenvalues in the presence of non-zero chemical potential $\mu$ one has to replace the (anti-)Hermitian Euclidean Dirac operator, see Eq.(10), with an operator of the following structure:

$$
\hat{D}_{\mu}=\left(\begin{array}{cc}
\mathbf{0} & i \hat{J}+\mu \mathbf{1} \\
i \hat{J}^{\dagger}+\mu \mathbf{1} & \mathbf{0}
\end{array}\right)
$$

$\ddagger$ Actually, the original paper 23 employed a slightly different but equivalent construction dealing with an "enlarged" internal space of the dimension $N+M$. We prefer to follow the general scheme because of its conceptual clarity. 
Such matrices are no longer (anti-)Hermitian and their eigenvalues go away from the (imaginary) axis to the complex plane. This phenomenon clearly bears some similarity to the effect of opening a closed system by converting its eigenvalues to resonances. As the result, the fermion determinant in the partition function (2) has a complex phase. On the level of true lattice simulations such a phase results in major computational difficulties. All this makes random matrix models a very useful tool for getting insights into gross universal features of the analytical structure of the QCD partition function. In turn, information on such a structure is based on the knowledge of statistics of complex eigenvalues of the Dirac operator with non-vanishing chemical potential which stimulated a lot of research of such eigenvalues [38, 39, 40, 41, 42, 43, 44.

Finally, it deserves to be briefly mentioned that objects related to weakly nonHermitian random matrices emerge also in the context of interesting problems of motion and localization of a quantum particle in disordered media subject to an imaginary vector potential [45] or a random scalar potential [46]. The first of these problems is related to the motion of superconductor flux lines in a sample with columnar defects, the second to the motion of a self-repelling polymer chain and other interesting problems (see the cited references). These developments clearly show that, apart from being a rich and largely unexplored mathematical object, weak nonHermitian deformations of random matrices enjoy direct physical applications and deserve to be studied in more detail.

The goal of the present paper is to give an overview of results and methods for dealing with spectra and eigenvectors of various non-Hermitian and nonunitary deformations of classical ensembles of random matrices. For the reasons which should be clear from our discussion above we concentrate on the regime of weak non-Hermiticity recognized in [47. It occurs when the imaginary parts of typical eigenvalues are comparable with the mean separation between neighbouring eigenvalues along the real axis. A simple analysis shows that the last condition is satisfied when the traces of the Hermitian matrix $H$ and the perturbing antiHermitian matrix $i \Gamma$ are related as: $\lim _{N \rightarrow \infty} \frac{N \operatorname{Tr} \Gamma^{2}}{\operatorname{Tr} H^{2}}<\infty$. We do not discuss at all interesting questions related to strongly non-Hermitian matrices, see e.g. papers 48 and references therein.

The first weakly non-Hermitian ensemble analysed in full generality [50] was that of non-Hermitian Gaussian deformations, with the anti-Hermitian part taken independently from the GUE. For this case one can develop a rigorous mathematical theory based on the method of orthogonal polynomials. Such a method allows one to study correlation properties of complex spectra to the same degree as is typical for earlier studied classes of random matrices [49. We briefly discuss the results obtained in this model in subsection 2.1 (the detailed exposition of the method and the derivation of the results can be found in [50]) as well as recent progress for other Gaussian ensembles and closely related models. We also introduce an alternative way of addressing the problem which places the correlation function of spectral determinants as primary object. This second method turns out to be indispensable when one deals with non-Gaussian deformations as those emerging in the theory of chaotic scattering. The latter problem was successfully studied in the work by Fyodorov and Khoruzhenko [51] and we discuss in subsection 2.2 the main ingredients of their solution. At the end of that subsection we indicate a close similarity between the results obtained for the Gaussian and for the finite-rank deformations.

Unfortunately, it is not known at the moment how to extend the exact methods to the case of non-Hermitian deformations of real symmetric matrices. The last question 
is of great practical interest for the application of the obtained results to chaotic scattering. Indeed, the majority of quantum chaotic systems possesses time-reversal invariance and hence should be adequately described by random matrices which are real symmetric [1. Restricting our attention to the simplest, still highly non-trivial statistical measure - the mean density of complex eigenvalues of the resulting complex symmetric effective Hamiltonian $\mathcal{H}_{\text {eff }}$-the answer can be given in a closed form [52]. The object turns out to be accessible via Efetov's supersymmetry approach which we shortly discuss in subsection 2.3.

Very recently the properties of bi-orthogonal eigenvectors of non-Hermitian random matrices attracted considerable interest of various researchers [53, 55, 55, 56. 57. In particular, an essential boost to these studies was given by the demonstrated relevance of the non-Hermitian effective Hamiltonian $\mathcal{H}_{\text {eff }}=\hat{H}-i \hat{\Gamma}$ to the problem of lasing in random media [54, 58, 59. Indeed, for systems of such a type the nonorthogonality of eigenmodes is known to play an important role 60. Similar effects were discussed in relation to the unimolecular decay in $\mathrm{NO}_{2}$ molecules 21]. In section 4 we discuss this issue for the regime of weak non-Hermiticity.

Most of our own results discussed in this review were reported earlier in the form of short communications [36, 51, 52, 57, 61, 62, 63] and in an unpublished paper by one of the authors 64. Some (but by far not all) details of the underlying derivations are elucidated in the present text, as far as space restrictions allow. In particular, appendix A contains the derivation of the formulas related to various aspects of the Itzykson-Zuber-Harish-Chandra integration over the unitary group extensively used in both [51] and 63.

\section{Non-Hermitian Deformations of Random Matrices}

\subsection{Gaussian deformations: from Wigner-Dyson to Ginibre eigenvalue statistics.}

In the present section we concentrate on a particular case of almost-Hermitian random matrices with i.i.d. entries $\hat{J}=\hat{H}+i v \hat{A}$ where $\hat{H}$ and $\hat{A}$ are taken independently from the Gaussian Unitary Ensemble (GUE) of Hermitian matrices $\hat{X}=\hat{X}^{\dagger}$ with probability density $\mathcal{P}(\hat{X})=Q_{N}^{-1} \exp \left(-N / 2 J_{0}^{2} \operatorname{Tr} \hat{X}^{2}\right)$.

Let us now introduce a new parameter $\tau=\left(1-v^{2}\right) /\left(1+v^{2}\right)$ and choose the scale constant $J_{0}^{2}$ to be equal to $(1+\tau) / 2$, for the sake of convenience. The parameter $\tau$ controls the magnitude of correlation between $J_{j k}$ and $J_{k j}:\left\langle J_{j k} J_{k j}\right\rangle=\tau / N$, hence the degree of non-Hermiticity. This is easily seen from the probability density function for our ensemble of the random matrices $\hat{J}$ :

$$
\mathcal{P}\left(\hat{J}, \hat{J}^{\dagger}\right)=C_{N}^{-1} \exp \left(-\frac{N}{\left(1-\tau^{2}\right)} \operatorname{Tr}\left(\hat{J} \hat{J}^{\dagger}-\tau \operatorname{Re} \hat{J}^{2}\right)\right),
$$

where $C_{N}=\left[\pi^{2}\left(1-\tau^{2}\right) / N^{2}\right]^{N^{2} / 2}$. All the $J_{j k}$ have zero mean and variance $\left\langle\left|J_{j k}\right|^{2}\right\rangle=1 / N$ and only $J_{j k}$ and $J_{k j}$ are pairwise correlated. If $\tau=0$ all the $J_{j k}$ are mutually independent and we have maximum non-Hermiticity. When $\tau$ approaches unity, $J_{j k}$ and $J_{k j}^{*}$ are related via $J_{j k}=J_{k j}^{*}$ and we are back to an ensemble of Hermitian matrices.

Our first goal is to determine the $n$-eigenvalue correlation functions in the ensemble of random matrices specified by Eq. (92). The density of the joint distribution 
of eigenvalues in this ensemble is given by

$\mathcal{P}_{N}(\{Z\})=c_{N}(\tau)|\Delta(\{Z\})|^{2} \exp \left(\frac{-N}{1-\tau^{2}} \sum_{j=1}^{N}\left[\left|Z_{j}\right|^{2}-\frac{\tau}{2}\left(Z_{j}^{2}+Z_{j}^{* 2}\right)\right]\right)$

where $c_{N}(\tau)=\frac{N^{N(N+1) / 2}}{\pi^{N} 1 ! \cdots N !\left(1-\tau^{2}\right)^{N / 2}},\{Z\}=\left(Z_{1}, \ldots, Z_{N}\right)$ is the vector of eigenvalues and $\Delta(\{Z\})=\prod_{j<k}\left(Z_{j}-Z_{k}\right)$ is the corresponding Vandermonde determinant.

To derive Eq. (10) we integrate $\mathcal{P}\left(\hat{J}, \hat{J}^{\dagger}\right)$ from Eq. (9) over the surface of all complex matrices whose eigenvalues are $Z_{1}, \ldots Z_{N}$. Following Dyson (49], p.501, see also 65]) we decompose every complex matrix with distinct eigenvalues as $\hat{J}=\hat{U}(\hat{Z}+\hat{R}) \hat{U}^{\dagger}$, where $\hat{Z}=\operatorname{diag}\left\{Z_{1}, \ldots Z_{N}\right\}, \hat{U}$ is a unitary matrix, and $\hat{R}$ is a strictly upper-triangular one. If we label the eigenvalues and require the first non-zero element in each column of $\hat{U}$ to be positive, then the decomposition is unique (it is frequently referred to as "Schur decomposition"). The Jacobian of the transformation $\hat{J} \rightarrow\{\hat{Z}, \hat{R}, \hat{U}\}$ depends only on $\hat{Z}$ and is given by the squared modulus of the Vandermonde determinant. So, integrating out $\hat{R}$ and $\hat{U}$ is straightforward and the resulting expression is Eq. (10).

The correlations between eigenvalues are conventionally characterized by the $n$ eigenvalue correlation functions. To define them we subdivide the vector of eigenvalues $\{Z\}=\left(Z_{1}, \ldots, Z_{N}\right)$ into two parts $\{z\}=\left(Z_{1}, \ldots, Z_{n}\right)$ and $\{\xi\}=\left(\xi_{1}, \ldots, \xi_{k}, \ldots, \xi_{N-n}\right)$ identifying $\xi_{k}=Z_{n+k}$. Then the correlation functions are given by:

$$
R_{n}(\{z\})=\frac{N !}{(N-n) !} \int d^{2}\{\xi\} \mathcal{P}_{N}\{\{z\},\{\xi\}\}
$$

where $d^{2}\{\xi\}=\prod_{l=1}^{N-n} d \operatorname{Re} \xi_{l} d \operatorname{Im} \xi_{l}$.

The form of the distribution Eq. (10) allows one to employ the powerful method of orthogonal polynomials [49]. Let $H_{n}(z)$ denote the $n$-th Hermite polynomial,

$$
H_{n}(z)=\frac{( \pm i)^{n}}{\sqrt{2 \pi}} \exp \left(\frac{z^{2}}{2}\right) \int_{-\infty}^{\infty} d t t^{n} \exp \left(-\frac{t^{2}}{2} \mp i z t\right) .
$$

The crucial observation borrowed from the paper [66 (see also the related paper [67]) is that the polynomials

$$
p_{n}(Z)=\frac{\tau^{n / 2} \sqrt{N}}{\sqrt{\pi} \sqrt{n !}\left(1-\tau^{2}\right)^{1 / 4}} H_{n}\left(\sqrt{\frac{N}{\tau}} Z\right)
$$

$n=0,1,2, \ldots$, are orthogonal in the complex plane $Z=X+i Y$ with the weight function

$$
w^{2}(Z)=\exp \left(-\frac{N}{\left(1-\tau^{2}\right)}\left[|Z|^{2}-\frac{\tau}{2}\left(Z^{2}+Z^{* 2}\right)\right]\right),
$$

i. e. $\int d^{2} Z p_{n}(Z) p_{m}\left(Z^{*}\right) w^{2}(Z)=\delta_{n m}$, where $d^{2} Z=d X d Y$.

The standard machinery of the method of orthogonal polynomials 49 yields the functions $R_{n}(\{z\})$ in the form

$$
R_{n}(\{z\})=\left.\operatorname{det}\left[K_{N}\left(Z_{j}, Z_{k}^{*}\right)\right]\right|_{j, k=1, \ldots, n},
$$

where the kernel $K_{N}\left(Z_{1}, Z_{2}^{*}\right)$ is given by

$$
K_{N}\left(Z_{1}, Z_{2}^{*}\right)=w\left(Z_{1}\right) w\left(Z_{2}^{*}\right) \sum_{n=0}^{N-1} p_{n}\left(Z_{1}\right) p_{n}\left(Z_{2}^{*}\right) .
$$


With Eqs. (13) -15] at hand, let us first examine the regime of strong nonHermiticity, i.e. the case when $\lim _{N \rightarrow \infty}(1-\tau)>0$, i.e. $\tau<1$ independent of $N$. In this regime the averaged density of eigenvalues $N^{-1} R_{1}(Z)$ is asymptotically zero outside the ellipse $[\operatorname{Re} Z /(1+\tau)]^{2}+[\operatorname{Im} Z /(1-\tau)]^{2} \leq 1$. Inside the ellipse $\lim _{N \rightarrow \infty} N^{-1} R_{1}(Z)=\left[\pi\left(1-\tau^{2}\right)\right]^{-1}$. This sets a microscopic scale on which the averaged number of eigenvalues in any domain of unit area remains finite when $N \rightarrow$ $\infty$. Remarkably, the $\tau$-dependence is essentially trivial on this scale: the statistical properties of eigenvalues are described by $\tilde{R}_{n}\left(z_{1}, \ldots, z_{n}\right) \equiv N^{-n} R_{n}\left(\frac{z_{1}}{\sqrt{N}}, \ldots, \frac{z_{n}}{\sqrt{N}}\right)$ and

$$
\lim _{N \rightarrow \infty} \tilde{R}_{n}\left(z_{1}, \ldots, z_{n}\right)=\left.\left[\frac{1}{\pi\left(1-\tau^{2}\right)}\right]^{n} e^{-\frac{1}{1-\tau^{2}} \sum_{j=1}^{n}\left|z_{j}\right|^{2}} \operatorname{det}\left[e^{\frac{1}{1-\tau^{2}} z_{j} z_{k}^{*}}\right]\right|_{j, k=1, \ldots, n} .
$$

This limiting relation can be inferred [50] from Mehler's formula for the Hermite polynomials 68. After the trivial additional rescaling $z \rightarrow z \sqrt{1-\tau^{2}}$ the expression on the right-hand side in Eq. (16) becomes identical to that found by Ginibre 69.

Now we move on to the regime of weak non-Hermiticity. We know that in this regime new non-trivial correlations occur on the scale: $\operatorname{Im} Z_{1,2}=O(1 / N)$, $\operatorname{Re} Z_{1}-\operatorname{Re} Z_{2}=O(1 / N)$. Correspondingly, we introduce new variables $x, y_{1}, y_{2}, \omega$ in such a way that: $x=\operatorname{Re}\left(Z_{1}+Z_{2}\right) / 2, y_{1,2}=N \operatorname{Im}\left(Z_{1,2}\right), \omega=N \operatorname{Re}\left(Z_{1}-Z_{2}\right)$, and consider them finite when performing the limit $N \rightarrow \infty$.

Substituting Eq. (12) into Eq. (15) and using the above definitions we can explicitly perform the limit $N \rightarrow \infty$, taking into account that $\lim _{N \rightarrow \infty} N(1-\tau)=\alpha^{2} / 2$. The details of the procedure are given elsewhere [50. In this regime

$$
\begin{aligned}
\lim _{N \rightarrow \infty} \frac{1}{N^{2}} K_{N}( & \left.+\frac{\omega / 2+i y_{1}}{N}, x-\frac{\omega / 2-i y_{2}}{N}\right)=\exp \left\{-\frac{y_{1}^{2}+y_{2}^{2}}{\alpha^{2}}+\frac{i x\left(y_{1}-y_{2}\right)}{2}\right\} \\
& \times \frac{1}{\pi \alpha} \int_{-\pi \nu_{s c}(x)}^{\pi \nu_{s c}(x)} \frac{d u}{\sqrt{2 \pi}} \exp \left[-\frac{\alpha^{2} u^{2}}{2}-u\left(y_{1}+y_{2}\right)+i \omega u\right]
\end{aligned}
$$

with $\nu_{s c}(X)=\frac{1}{2 \pi} \sqrt{4-X^{2}}$ standing for the Wigner semicircular density of real eigenvalues of the Hermitian part $\hat{H}$ of the matrices $\hat{J}$.

The kernel $K_{N}$ given by Eq. (17) determines all the properties of complex eigenvalues in the regime of weak non-Hermiticity. For instance, the mean value of the density $\rho(Z)=\sum_{i=1}^{N} \delta^{(2)}\left(Z-Z_{i}\right)$ of complex eigenvalues $Z=X+i Y$ is given by $\langle\rho(Z)\rangle=K_{N}\left(Z, Z^{*}\right)$, i.e. by putting $y_{1}=y_{2}$ and $\omega=0$ in Eq. (17) (cf. [47] found by the supersymmetry approach described in subsection 2.3).

One of the most informative statistical measures of the spectral correlations is the 'connected' part of the two-point correlation function of eigenvalue densities:

$$
\left\langle\rho\left(Z_{1}\right) \rho\left(Z_{2}\right)\right\rangle_{c}=\left\langle\rho\left(Z_{1}\right)\right\rangle \delta^{(2)}\left(Z_{1}-Z_{2}\right)-\mathcal{Y}_{2}\left(Z_{1}, Z_{2}\right),
$$

In particular, it determines the variance $\Sigma^{2}(D)=\left\langle n(D)^{2}\right\rangle-\langle n(D)\rangle^{2}$ of the number $n=\int_{D} d^{2} Z \rho(Z)$ of complex eigenvalues in any domain $D$ in the complex plane as:

$$
\begin{aligned}
& \Sigma_{2}(D)=\int_{D} d^{2} Z_{1} \int_{D} d^{2} Z_{2}\left[\left\langle\rho\left(Z_{1}\right) \rho\left(Z_{2}\right)\right\rangle-\left\langle\rho\left(Z_{1}\right)\right\rangle\left\langle\rho\left(Z_{2}\right)\right\rangle\right] \\
& =\int_{D} d^{2} Z\langle\rho(Z)\rangle-\int_{D} d^{2} Z_{1} \int_{D} d^{2} Z_{2} \mathcal{Y}_{2}\left(Z_{1}, Z_{2}\right)
\end{aligned}
$$

Comparing Eq. (18) with the definitions Eqs. (13 15) we see that the cluster function $\mathcal{Y}_{2}\left(Z_{1}, Z_{2}\right)$ is expressed in terms of the kernel $K_{N}$ as $\mathcal{Y}_{2}\left(Z_{1}, Z_{2}\right)=$ $\left|K_{N}\left(Z_{1}, Z_{2}^{*}\right)\right|^{2}$. 
It is evident that in the limit of weak non-Hermiticity the kernel $K_{N}$ depends on $X$ only via the semicircular density $\nu_{s c}(X)$. Thus, it does not change with $X$ on the local scale comparable with the mean spacing along the real axis $\Delta \sim 1 / N$.

The cluster function is given by the following explicit expression:

$\mathcal{Y}\left(\omega, y_{1}, y_{2}\right)=\frac{N^{4}}{\pi^{2} \alpha^{2}} e^{-2 \frac{y_{1}^{2}+y_{2}^{2}}{\alpha^{2}}}\left|\int_{-\pi \nu(X)}^{\pi \nu(X)} \frac{d u}{(2 \pi)^{1 / 2}} \exp \left[-\frac{\alpha^{2} u^{2}}{2}-u\left(y_{1}+y_{2}\right)+i u \omega\right]\right|^{2}$

The parameter $a=\pi \nu(X) \alpha$ controls the deviation from Hermiticity $\S$. When $a \rightarrow 0$ the cluster function tends to GUE form $\mathcal{Y}_{2}\left(\omega, y_{1}, y_{2}\right)=\frac{N^{4}}{\pi^{2}} \delta\left(y_{1}\right) \delta\left(y_{2}\right) \frac{\sin ^{2} \pi \nu(X) \omega}{\omega^{2}}$. In the opposite case $a \gg 1$ we expect it to match with the regime of strong nonHermiticity emerging for $\alpha \sim N^{1 / 2}$. To verify this we notice that for large $\alpha^{2}$ the integral in Eq. (20) can be evaluated by the saddle-point method. The saddle-point in that case is $u_{s}=-\left(y_{1}+y_{2}-i \omega\right) / \alpha^{2}$ and to the leading order the result is non-vanishing as long as $\left|\operatorname{Re} u_{s}\right| \leq \pi \nu_{s c}(X)$ that is $\left|y_{1}+y_{2}\right| \leq \pi \nu_{s c}(X) \alpha^{2}$. Assuming that this is the case one performs the Gaussian integration around $u_{s}$ and finds (in the original variables $Z_{1}, Z_{2}$ ) the expression equivalent (up to a trivial rescaling) to that found by Ginibre [69]: $\mathcal{Y}_{2}\left(Z_{1}, Z_{2}\right)=\left(N^{2} / \pi \alpha^{2}\right)^{2} \exp \left\{-N^{2}\left|Z_{1}-Z_{2}\right|^{2} / \alpha^{2}\right\}$. Remembering $\nu_{s c}(X)=\frac{1}{2 \pi} \sqrt{4-X^{2}}$ and $\alpha^{2}=N v^{2}$ and performing the same procedure for the mean density $\rho(Z)=K\left(Z, Z^{*}\right)$ one finds that the condition for the saddlepoint discussed above just ensures that both $Z_{1}$ and $Z_{2}$ are inside an elliptic blob $(\operatorname{Re} Z)^{2} / 4+(2 \operatorname{Im} Z)^{2} / v^{2}=1$ in the complex plane filled in uniformly with eigenvalues with the density $\rho(Z)=N /\left(\pi v^{2}\right)$, in agreement with [70. This is basically the case of "strong" non-Hermiticity, see the discussion preceeding Eq.16), and the "elliptic" law is believed to be the common feature for strongly non-Hermitian matrices with independent entries 65, 71.

One can further calculate the Fourier transform of the cluster function over its arguments $\omega, y_{1}, y_{2}$ and find an explicit expression for the spectral form-factor. It allows one to determine the variance $\Sigma_{2}$ of a number of eigenvalues in any domain $D$ of the complex plane. In this way one traces, in particular, a tendency for gradual decorrelation of real parts of the eigenvalues with growing non-Hermiticity. Similarly, one can investigate various regimes of eigenvalue repulsion in the complex plane by looking at small-distance behaviour of the nearest neighbour distance distribution. All these calculations are presented and thoroughly discussed in [50].

Further calculations in the framework of the present model were done recently by Akemann [42. Motivated by QCD applications the author first suggested to consider the matrix integral

$\mathcal{Z}\left(\left\{m_{f}\right\}\right)=\int d \hat{J} d \hat{J}^{\dagger} \mathcal{P}\left(\hat{J}, \hat{J}^{\dagger}\right) \prod_{f=1}^{N_{F}}\left[\operatorname{det}\left(\hat{J}-i m_{f} \mathbf{1}\right) \operatorname{det}\left(\hat{J}^{\dagger}+i m_{f} \mathbf{1}\right)\right]$

with $\mathcal{P}\left(\hat{J}, \hat{J}^{\dagger}\right)$ being the joint probability density function of the random matrices $\hat{J}$, see Eq.(9). This integral is a model for the Euclidean QCD partition function in 3 dimensions at nonzero chemical potential, when the chiral structure of the Dirac operator is irrelevant. The latter fact gives some justification of the purely phenomenological model based on Eq.(21). On the other hand, the matrix integral is interesting by itself and its calculation is very intimately related to calculating the eigenvalue correlation function Eq.(11).

$\S$ In our earlier Letter [50] we used the definition of the parameter $a$ different by a factor of 2 from the present one. 
Indeed, having in mind the decomposition $\left(Z_{1}, \ldots, Z_{N}\right)=(\{z\},\{\xi\})$ as in Eq.(11) and noticing that the Vandermonde determinant $\Delta(\{Z\})$ can be rewritten as :

$$
\Delta(\{Z\})=\Delta(\{z\}) \Delta(\{\xi\}) \prod_{i=1}^{n} \prod_{j=1}^{N-n}\left(Z_{i}-\xi_{j}\right)
$$

it is easy to see that the correlation function $R_{n}(\{z\})$ as defined in Eq. (11) can be put in the form:

$R_{n}(\{z\})=\frac{c_{n}(\tau)}{c_{N-n}(\tau)}|\Delta(\{z\})|^{2}\left[\prod_{i=1}^{n} w^{2}\left(Z_{i}\right)\right] \int d^{2}\{\xi\} \mathcal{P}_{N-n}(\{\xi\}) \prod_{i=1}^{n}\left|\prod_{j=1}^{N-n}\left(Z_{i}-\xi_{j}\right)\right|^{2}$

where $\mathcal{P}_{N-n}(\{\xi\})$ is just the density of joint probability of eigenvalues $\xi_{1}, \ldots ., \xi_{N-n}$ of a random Gaussian non-Hermitian matrix $J_{N-n}=H_{1}+i v A$ of the size $(N-n) \times(N-n)$. But the integral in the last equation is just the expectation value of the product of determinants:

$$
\left\langle\prod_{i=1}^{n}\left[\operatorname{det}\left(Z_{i} \mathbf{1}-\hat{J}_{N-n}\right) \operatorname{det}\left(Z_{i}^{*} \mathbf{1}-\hat{J}_{N-n}^{\dagger}\right)\right]\right\rangle_{J_{N-n}}
$$

Thus, we see that the problem of evaluating the multipoint correlation function $R_{n}(\{z\})$ of complex eigenvalues is, in fact, equivalent to calculating the RMT partition function for $\mathrm{QCD}_{3}$, Eq. 21). Akemann 42] used this observation to express the partition function $\mathcal{Z}$ in terms of the $R_{n}(\{z\})$, and in this way exploited the orthogonal polynomial representation, see Eqs.(1415).

Here we would like to note that by reversing this kind of reasoning one could get access to the multipoint correlations of complex eigenvalues if one were able to provide an independent technique of evaluating $\mathcal{Z}$. This was realized by Fyodorov and Khoruzhenko [51] who implemented such an idea for a more general class of ensembles. We will see below, that a reduction similar to that discussed above holds beyond the Gaussian case, provided one deals with the regime of weak non-Hermiticity. Moreover, it will be shown how to perform the ensemble average of the product of characteristic polynomials for an arbitrary fixed non-Hermitian deformation by mapping the analogue of partition function Eq.(21) to a fermionic version of a nonlinear $\sigma$-model.

Let us discuss briefly other types of (weakly) non-Hermitian deformations based on the Gaussian case and studied by various authors. Recently Akemann 43 . considered a model with the joint probability density of variables $Z_{i}$ defined by

$\mathcal{P}_{N}^{(c h)}(\{Z\}) \propto\left|\Delta\left(\{Z\}^{2}\right)\right|^{2}\left|Z_{1} \ldots Z_{N}\right|^{2 a+1} \exp \left\{\frac{-N}{1-\tau^{2}} \sum_{j=1}^{N}\left[\left|Z_{j}\right|^{2}-\frac{\tau}{2}\left(Z_{j}^{2}+Z_{j}^{* 2}\right)\right]\right\}(23)$

The new factors $|Z|^{2 a+1}, a>-1$ as well as squared variables inside the Vandermonde determinant were introduced in an ad hoc way to make this ensemble a candidate for chiral extension of the Gaussian case, provided one interpretes the variables $Z$ as complex Dirac eigenvalues. In such an interpretation the parameter $a$ can be related to the number of massless quark flavours $N_{F}$, whereas the chemical potential is phenomenologically identified with $\frac{1}{2} \sqrt{1-\tau^{2}}$. The advantage of the model is that it still can be solved by the orthogonal polynomial method, with (properly normalized) Laguerre polynomials of complex argument $L_{k}^{(a)}\left(\frac{N z^{2}}{2 \tau}\right)$ replacing the 
Hermite polynomials. In particular, elegant explicit expressions for the limiting form of the kernel $K^{(a)}\left(Z_{1}, Z_{2}^{*}\right)$ were derived both in the strong non-Hermiticity and the weak non-Hermiticity regimes [43]. On the other hand, the main drawback of the model is its ad-hoc construction in terms of the variables $Z_{i}$. In particular, it is completely unclear how to construct the corresponding random matrix model for the Dirac operator which could have the starting formula (23) as the joint probability density of its eigenvalues. The hope is, nevertheless, that the universality will again make the eigenvalue correlations insensitive to the particular choice of the underlying model 44. However, we believe that the full analysis of the random matrix model Eq.(8) in the regime of weak non-Hermiticity still remains an important unsolved problem.

In the spirit of the methods discussed in the present section one can efficiently study Gaussian ensembles corresponding to the weakly non-Hermitian quaternion random matrices. Matrices with such (symplectic) symmetry are also important for QCD applications [39] and that fact inspired quite a few attempts of understanding their properties analytically $72,73,74,75]$. The most complete solution was presented by Kanzieper [75] who used the skew orthogonal polynomials.

Recently another interesting extension of the model Eq.(9) was suggested by Garcia-Garcia et al. [6]. The authors introduced an ensemble that interpolates between the GUE, the Ginibre ensemble of strongly non-Hermitian matrices and the Hermitian Poisson ensemble with uncorrelated real eigenvalues. The physical motivation behind such a generalization comes from the wish to understand better an interplay between the effects of non-Hermiticity and the effects of Anderson localization which drive the statistics of real eigenvalues towards an uncorrelated Poisson spectrum. It is claimed that the model can adequately describe critical spectral statistics of open disordered systems close to the Anderson transition. The authors managed to provide a closed analytical expression for the eigenvalue correlations in various regimes and concluded that critical statistics is not modified by weak non-Hermiticity effects. They further put forward an expectation that opening a critical system does not affect the multifractal dimension of the wavefunction. Whether it is indeed the case remains to be verified in numerical simulations.

\subsection{Finite-rank deformations of Hermitian matrices: the case of chaotic scattering}

As it was discussed in the introduction, the poles of the $S$-matrix (resonances) are just the complex eigenvalues of an effective random matrix Hamiltonian $\mathcal{H}_{\text {eff }}=\hat{H}-i \hat{\Gamma}$. The requirement of the $S$-matrix unitarity and causality restricts $\hat{\Gamma}$ to be of the rank $M$, characterized by $M$ positive eigenvalues $\gamma_{c}>0, c=1, \ldots, M$, the rest $N-M$ eigenvalues being identically zero. To satisfy the condition of weak non-Hermiticity for the case of a few open channels: $N \gg M \sim 1$ means just to consider these eigenvalues to be $N$-independent: $\gamma_{c}<\infty$. The condition ensures the width $\Gamma_{k}$ of a typical resonance to be comparable with the mean separation $\Delta$ between neighbouring resonances along the real axis.

Despite quite substantial efforts [14, 15, 11, 52, 61] non-perturbative results on resonance statistics for few-channel scattering were very restricted for a long time. The knowledge amounted mainly to (i) the joint probability density of all resonances for the system with a single open channel and Gaussian-distributed transition amplitudes by Sokolov et al. $\hat{W}[13$ and (ii) the mean density of $S$-matrix poles for arbitrary $M \ll N$ [1] 52. A systematic analytical approach to the statistical properties of 
resonances for systems with broken time-reversal invariance was suggested in [51. In what follows we discuss that approach in more detail.

For the sake of notational convenience we have changed the sign in front of $\Gamma \|$. This trivially amounts to changing the signs of imaginary parts of all eigenvalues in the resulting expressions. From now on we consider an ensemble of random $N \times N$ complex matrices $J=H+i \Gamma$, where $H$ is $N \times N$ matrix taken from a Gaussian Unitary Ensemble (GUE) of Hermitian matrices with the probability density $\mathcal{P}(H) \propto \exp \left(-\frac{N}{2} \operatorname{tr} H^{2}\right), H=H^{\dagger}$. As for the matrix $\Gamma=\Gamma^{\dagger}$, we consider it to be a fixed nonnegative one: $\Gamma \geq 0$.

By analogy with the complex numbers,

$$
H=\frac{1}{2}\left(J+J^{\dagger}\right) \equiv \operatorname{Re} J ; \quad \Gamma=\frac{1}{2 i}\left(J-J^{\dagger}\right) \equiv \operatorname{Im} J
$$

with the "only" difference that $\operatorname{Re} J$ and $\operatorname{Im} J$ do not commute. Then the probability density function in our ensemble of random matrices $J$ can be written in the form

$$
\mathcal{P}(J) \propto e^{-\frac{N}{2} \operatorname{Tr}(\operatorname{Re} J)^{2}} \delta(\Gamma-\operatorname{Im} J) .
$$

with a matrix $\delta$-function for Hermitian matrices. We do not specify the multiplicative constant in Eq. (24). It can be found from the normalization condition. Similarly, and by the same reason, we will systematically disregard multiplicative constants when dealing with probability densities and correlation functions.

Eq. (24) can be used to obtain the density of joint distribution of eigenvalues by integrating $\mathcal{P}(J)$ over the degrees of freedom that are complementary to the eigenvalues of $J$. This again can be done following Dyson's method (see preceding section). To perform the integration over upper triangular matrices $R$ it is technically convenient to use a Fourier-integral representation for the $\delta$-function in Eq. (24). This reduces the corresponding integral to a Gaussian one and after quite straightforward algebraic manipulations the resulting expression is

$$
P_{N}(Z) \propto e^{-\frac{N}{2} \operatorname{Re} \operatorname{Tr} Z^{2}-\frac{N}{2} \operatorname{Tr} \Gamma^{2}}|\Delta(Z)|^{2} Q_{M}(\operatorname{Im} Z),
$$

where $Q_{M}(\operatorname{Im} Z)$ is the remaining integral

$$
Q_{M}(\operatorname{Im} Z)=\int[d U] \prod_{l=1}^{N} \delta\left(\operatorname{Im} z_{l}-\left(U^{\dagger} \Gamma U\right)_{l l}\right),
$$

over the unitary group $U(N)$, $[d U]$ being the Haar measure. It is clear that we can consider $\Gamma$ to be diagonal, and the integral Eq.(26), has an obvious interpretation of the joint probability density of diagonal entries for a matrix with prescribed set of eigenvalues $\Gamma$, randomly rotated by a unitary matrix $U$. Recently this interpretion was helpful in applications to the problem of Wigner time-delay distribution 77 .

To proceed further we need to integrate over $U$. Again it is convenient to use the Fourier-integral representation for the $\delta$-functions in Eq. (26):

$$
Q_{M}(\operatorname{Im} Z)=\int \frac{d K}{(2 \pi)^{N}} e^{i \operatorname{Im} \operatorname{Tr} K Z} \int[d U] e^{-i \operatorname{Tr} K U^{\dagger} \Gamma U},
$$

where the first integration is over all real diagonal matrices $K$ of dimension $N, d K$ being $d k_{1} \ldots d k_{N}$.

\| in the rest of this subsection we will denote matrices without "hat" 
When the eigenvalues $\gamma_{1}, \ldots, \gamma_{N}$ of $\Gamma$ are all distinct the integration over $U(N)$ can be performed using the famous Itzykson-Zuber-Harish-Chandra (IZHC) formula [78]:

$$
\int[d U] e^{-i \operatorname{Tr} K U^{\dagger} \Gamma U}=\frac{\operatorname{det}\left[e^{-i k_{n} \gamma_{m}}\right]_{n, m=1, \ldots, N}}{\Delta(-i\{K\}) \Delta(\{\gamma\})}
$$

where $\{K\}=\operatorname{diag}\left(K_{1}, \ldots, K_{N}\right)$ and $\{\gamma\}=\operatorname{diag}\left(\gamma_{1}, \ldots, \gamma_{N}\right)$ and we have chosen the total volume of $U(N)$ equal to 1 . We, however, are mostly interested in the case when $\Gamma$ has a small rank $M \ll N$, i.e. it has only $M$ nonzero eigenvalues which we denote by $\gamma_{1}, \ldots, \gamma_{M}$, the rest $N-M$ being zero. This limit of highly degenerate eigenvalues is difficult to perform in the original IZHC formula. A way to circumvent this difficulty is discussed in the appendix A1 where it is shown that:

$$
\int[d U] e^{-i \operatorname{Tr} K U^{\dagger} \Gamma U}=i^{-N^{2}} M ! \frac{\operatorname{det} \gamma^{M-N}}{\Delta(\gamma)} \int d \Lambda \Delta(\Lambda) e^{-i \sum_{c=1}^{M} \gamma_{c} \lambda_{c}} \prod_{j=1}^{N} \prod_{c=1}^{M} \frac{1}{\lambda_{c}-k_{j}}
$$

with $\Lambda=\operatorname{diag}\left(\lambda_{1}, \ldots, \lambda_{M}\right)$. Here it is meant that the integration contour is chosen in such a way that $\operatorname{Im} \lambda_{c}=0^{+}$.

To perform the integration over $K$ is a simple task with the use of the identities:

$$
\prod_{c=1}^{M} \frac{1}{\lambda_{c}-k_{j}}=\sum_{c=1}^{M} \frac{1}{\lambda_{c}-k_{j}} \prod_{s(\neq c)} \frac{1}{\lambda_{c}-\lambda_{s}} ; \int_{-\infty}^{\infty} \frac{d k}{2 \pi} \frac{e^{i \operatorname{Im} z_{j} k}}{\lambda_{c}-k}=\left\{\begin{array}{cl}
-i e^{i \operatorname{Im} z_{j} \lambda_{c}} & \operatorname{Im} z_{i}>0 \\
0 & \text { otherwise }
\end{array}\right.
$$

Taking into account that due to the specific structure of the matrices $J$ their eigenvalues lie in the upper part of the complex plane we assume in all formulae below that $\operatorname{Im} z_{j}>0$ for all $j$. This finally results in:

$Q_{M}(\operatorname{Im} Z) \propto M ! \frac{\operatorname{det} \gamma^{M-N}}{\Delta(\gamma)} \int_{R^{M}} d \Lambda e^{-i \sum_{c=1}^{M} \gamma_{c} \lambda_{c}} \Delta(\Lambda) \prod_{j=1}^{N} \sum_{q=1}^{M} \frac{e^{i \lambda_{q} \operatorname{Im} z_{j}}}{\prod_{s(\neq q)}\left(\lambda_{q}-\lambda_{s}\right)}$.

The pair of equations (25130) provides an explicit representation for the joint probability density of $N$ resonances $z_{j}$ in the complex plane. For the particular case of a non-Hermitian rank-one perturbation of GUE matrices (single-channel case $M=1$ ) the density of joint probability of all complex eigenvalues was found earlier by Stöckmann and Seba for a closely related model of random couplings $\hat{W}[13$. Their result follows from our Eq. (25)301) by noticing (i) that for $M=1$ the $\lambda$-integration is trivial to perform, yielding

$$
\left.\mathcal{P}_{N}(Z)\right|_{M=1} \propto e^{-\frac{N}{2} \operatorname{Re} \operatorname{Tr} Z^{2}}|\Delta(Z)|^{2} \frac{e^{-N \gamma^{2} / 2}}{\gamma^{N-1}} \delta\left(\gamma-\sum_{j=1}^{N} \operatorname{Im} z_{j}\right)
$$

and (ii) in their case the only nonzero eigenvalue $\gamma>0$ is a random variable with the probability density $\mathcal{P}(\gamma) \propto \gamma^{N-1} \exp -\left[N \gamma / \gamma_{0}\right]$.

Our main goal is to use equations (25130) as a basis for calculating the $n$-eigenvalue correlation functions $R_{n}(\{z\})$ as defined in Eq. (11). In what follows we will calculate $R_{n}(\{z\})$ for arbitrary fixed $n, M$ in the limit $N \rightarrow \infty$, but it may be instructive for the reader first to verify all the steps on the simplest case $M=1$, starting from the Eq. (31) and following [51]. On the first stage we will replace the integration over $\xi$ in (11) by averaging over the ensemble of non-Hermitian random matrices $J_{N-n}=H_{N-n}+i \Gamma$, 
with $H_{N-n}$ being a GUE matrix of the reduced size $(N-n) \times(N-n)$ (cf. Eq.(22) $)$. In this way one finds the following representation for the correlation functions:

$R_{n}(\{z\}) \propto \frac{C_{\gamma}(\{z\})}{\operatorname{det} \gamma^{n}}|\Delta(\{z\})|^{2} e^{-\frac{N-n}{2} \sum_{j=1}^{n} \operatorname{Re} z_{j}^{2}} \prod_{j=1}^{n} \sum_{k=1}^{M} \frac{e^{-2 y_{j} g_{k}}}{\prod_{s(\neq k)}\left(g_{k}-g_{s}\right)}$

where we introduced the notation $g_{c}=\frac{1}{2}\left(\gamma_{c}+\gamma_{c}^{-1}\right)$ and denoted:

$$
C_{\tilde{\gamma}}(\{z\})=\left\langle\prod_{j=1}^{n}\left|\operatorname{det}\left[z_{j}-J_{N-n}(\tilde{\gamma})\right]\right|^{2}\right\rangle_{G U E}
$$

The corresponding derivation is presented in appendix $\mathrm{B}$ and is valid in the limit $n, M \ll N \rightarrow \infty$.

Thus, the problem amounts to evaluation of the correlation function of the determinants in Eq. (33). To proceed we first write each of the determinants as a Gaussian integral over a set of Grassmann variables $\chi, \chi^{\dagger}$ :

$$
\prod_{p=1}^{n}\left|\operatorname{det}\left[z_{p}-J_{N-n}(\gamma)\right]\right|^{2}=\int d \chi d \chi^{\dagger} e^{-\left(\chi^{\dagger} \mathbf{Z}_{2 n} \chi+\chi^{\dagger} \mathbf{H}_{N-n} \chi+i \chi^{\dagger}\left[\Gamma \otimes \mathbf{L}_{2 n}\right] \chi\right)} .
$$

When this is done, the GUE average over $H_{N-n}$ becomes trivial and yields the terms quartic with respect to the Grassmannian vectors. These terms can be further traded for an auxilliary integration over a Hermitian matrix $S$ of the size $2 n \times 2 n$ (the so-called Hubbard-Stratonovich transformation). Then the integration over the Grassmann fields becomes again Gaussian and is trivially performed yielding again a determinant. Introducing diagonal matrices defined as: $\mathbf{Z}_{2 n}=\operatorname{diag}\left(\{z\},\{z\}^{\dagger}\right)$ and $\mathbf{L}_{2 n}=\operatorname{diag}\left(\mathbf{1}_{n},-\mathbf{1}_{n}\right)$ and using the finite-rank property of $\Gamma$ one easily brings the result to the following form:

$C_{\gamma}(\{z\})=\int[d S] e^{-(N-n) \operatorname{Tr}\left[\frac{1}{2} S^{2}-\ln \left(\mathbf{Z}_{2 n}-i S\right)\right]} \prod_{c=1}^{M} \operatorname{det}\left[\mathbf{1}_{2 n}+i \gamma_{c} \mathbf{L}_{2 n}\left(\mathbf{Z}_{2 n}-i S\right)^{-1}\right]$

We are interested in evaluating the above integrals in the limit $N \rightarrow \infty$. Let us now recall that nontrivial eigenvalue correlations are expected to occur on such a scale when the eigenvalues are separated by distances comparable with the mean eigenvalue separation for GUE matrices $H$ (see preceding section), the latter distance being of the order $(N-n)^{-1}$ for our choice of $\mathcal{P}(H)$. Accordingly, it is convenient to separate the "center of mass" coordinate $x=\frac{1}{n} \sum_{j=1}^{n} \operatorname{Re} z_{j}$ so that $z_{j}=x+\frac{\tilde{z}_{j}}{N-n}$, where both the real and imaginary parts of $\tilde{z}_{j}$ are of the order of unity in the limit when $N \rightarrow \infty$ and $M$ is fixed. In this limit $R_{n}(\{z\})$ is effectively a function of $\{\tilde{z}\}$ ( $x$ is fixed) which we are going to calculate. To this end, we expand the logarithm in the exponent of Eq.(35) as:

$$
(N-n) \operatorname{Tr} \ln \left(\mathbf{Z}_{2 n}-i S\right) \approx(N-n) \operatorname{Tr} \ln \left(x \mathbf{1}_{2 n}-i S\right)+\operatorname{Tr}\left[\tilde{\mathbf{Z}}_{2 n}\left(x \mathbf{1}_{2 n}-i S\right)^{-1}\right]+\ldots
$$

keeping only terms up to the order $O(1)$ when $N \rightarrow \infty$. With the same precision we set $\tilde{\mathbf{Z}}_{2 n}=x \mathbf{1}_{2 n}$ in the pre-exponential determinant factor of the integrand, Eq.(35).

The next step towards evaluating the integral in (35) is to diagonalize the Hermitian matrix $S$ as: $S=U_{2 n} \Sigma U_{2 n}^{-1}$, where $U_{2 n} \in U(2 n)$ and $\Sigma=\operatorname{diag}\left(\sigma_{1}, \ldots, \sigma_{2 n}\right)$. Then, keeping only the terms relevant in the limit $N \rightarrow \infty$, we obtain:

$$
C_{\gamma}(\{z\})=\int d \Sigma \Delta^{2}(\Sigma) e^{-(N-n) \sum_{k=1}^{2 n}\left[\frac{\sigma_{k}^{2}}{2}-\ln \left(x-i \sigma_{k}\right)\right]}\langle C(S)\rangle_{U(2 n)}
$$


where

$$
\langle C(S)\rangle_{U(2 n)}=\int\left[d U_{2 n}\right] e^{-\operatorname{Tr}\left[\tilde{\mathbf{Z}}_{2 n}\left(x \mathbf{1}_{2 n}-i S\right)^{-1}\right]} \prod_{c=1}^{M} \operatorname{det}\left[\mathbf{1}_{2 n}+i \gamma_{c} \mathbf{L}_{2 n}\left(x \mathbf{1}_{2 n}-i S\right)^{-1}\right](38)
$$

The form of the integrand in (37) suggests exploiting the saddle-point method in the integral over $\sigma_{k}, k=1, \ldots, 2 n$. Altogether there are $2^{2 n}$ saddle-points: $\sigma_{k}^{(s)}=-\frac{i}{2}\left(x+i \epsilon_{k} \sqrt{4-x^{2}}\right)$ each corresponding to a particular choice of $2 n$ signs $\epsilon_{k}= \pm 1$. It is easy to understand, however, that not all these saddle points are equally important in the limit of large $N$. The leading order contribution comes from integration around those saddle-points where exactly $n$ parameters $\epsilon_{k}$ equal 1 (the rest being equal -1). All other choices can be neglected as they lead to lower order terms. This is because of the presence of the Vandermonde determinant in the integrand:

$$
\Delta^{2}(\Sigma)=\prod_{k_{1}<k_{2}}\left(\sigma_{k_{1}}-\sigma_{k_{2}}\right)^{2}=\prod_{k_{1}<k_{2}}\left(\delta \sigma_{k_{1}}-\delta \sigma_{k_{2}}+\frac{1}{2}\left(\epsilon_{k_{1}}-\epsilon_{k_{2}}\right) \sqrt{4-x^{2}}\right)^{2}
$$

where we denoted by $\delta \sigma_{k}$ fluctuations around a given saddle-point to be treated in the Gaussian approximation. It is now evident, that we should require that the minimum number of the differences $\epsilon_{k_{1}}-\epsilon_{k_{2}}, \quad k_{1}<k_{2}=1,2, \ldots, n$ vanish. Otherwise, the Vandermonde determinant is proportional to higher powers of $\delta \sigma_{k}$, each power producing a small factor $(N-n)^{-1}$ when integrating around the saddle-point in the Gaussian approximation. This requirement fixes the choice of the saddle-points described above.

At the same time, all relevant saddle-points are equivalent (because they can be transformed one to the other by an element of the $U(2 n)$ group) and produce the same contribution. Therefore, we can take one of them, suppressing the multiplicative constants as usual. Our choice of $\Sigma^{(s)}$ is $\Sigma^{(s)}=-\frac{i}{2} x \mathbf{1}_{2 n}+\pi \nu(x) \mathbf{L}_{2 n}$, where the symbol $\nu(x)$ stands for the semicircular density of real eigenvalues of the matrices $H$, $\nu(x)=\frac{1}{2 \pi} \sqrt{4-x^{2}}$. Substituting $\Sigma^{(s)}$ to the integrand it is easy to verify that in the limit $N \rightarrow \infty$ we have:

$$
C_{\gamma}(\{z\}) \propto e^{\frac{N-n}{2} \sum_{j=1}^{n} \operatorname{Re} z_{j}^{2}} C_{\gamma}^{s}(\{\tilde{z}\})
$$

where $\{\tilde{z}\}=\left(\tilde{z}_{1}, \ldots, \tilde{z}_{n}\right), \tilde{z}_{j}=(N-n)\left(z_{j}-x\right), j=1, \ldots, n$, and

$C_{\gamma}^{s}(\{\tilde{z}\})=\int\left[\mathrm{d} \mathbf{Q}_{2 n}\right] e^{-i \pi \nu(x)} \operatorname{Tr} \tilde{\mathbf{Z}}_{2 n} \mathbf{Q}_{2 n} \prod_{c=1}^{M} \operatorname{det}\left[\mathbf{1}_{2 n}+\frac{i \gamma_{c} x}{2} \mathbf{L}_{2 n}+\pi \nu(x) \gamma_{c} \mathbf{L}_{2 n} \mathbf{Q}_{2 n}\right]$

In (40) $\mathbf{Q}_{2 n}=U_{2 n}^{-1} \mathbf{L}_{2 n} U_{2 n}$ and the integration is over the coset space $U(2 n) / U(n) \otimes$ $U(n)$.

Thus, the problem reduces to evaluation of an integral over a coset space. This type of integrals is known in the literature under the name of zero-dimensional nonlinear $\sigma$-models and the corresponding calculation is a standard one and is outlined in [51]. The result is given by:

$$
\begin{gathered}
C_{\gamma}^{s}(\{\tilde{z}\}) \propto \frac{\operatorname{det} \gamma^{n}}{|\Delta(\{\tilde{z}\})|^{2}} \int_{-1}^{1} d \lambda_{1} \ldots \int_{-1}^{1} d \lambda_{n} \operatorname{det}\left[e^{-i \pi \nu(x) \tilde{z}_{j} \lambda_{k}}\right] \operatorname{det}\left[e^{i \pi \nu(x) \tilde{z}_{j}^{*} \lambda_{k}}\right] \prod_{j=1}^{n} \prod_{c=1}^{M}\left[g_{c}+\pi \nu(x) \lambda\right] \\
\propto \frac{n ! \operatorname{det} \gamma^{n}}{|\Delta(\{\tilde{z}\})|^{2}} \operatorname{det}\left[\int_{-1}^{1} d \lambda \prod_{c=1}^{M}\left[g_{c}+\pi \nu(x) \lambda\right] e^{i \pi \nu(x) \lambda\left(\tilde{z}_{j}-\tilde{z}_{k}^{*}\right)}\right]
\end{gathered}
$$


Combining this with Eqs. (32) and (39), denoting $u=\lambda \pi \nu(x)$ and restoring the normalization we finally see that the correlation functions have the following simple structure:

$$
\frac{1}{N^{2 n}} R_{n}\left(x+\frac{\tilde{z}_{1}}{N}, \ldots, x+\frac{\tilde{z}_{n}}{N}\right)=\left.\operatorname{det}\left[K\left(\tilde{z}_{j}, \tilde{z}_{k}^{*}\right)\right]\right|_{j, k=1, \ldots, n},
$$

where the kernel $K\left(\tilde{z}_{j}, \tilde{z}_{k}^{*}\right)$ is given by

$$
K\left(\tilde{z}_{1}, \tilde{z}_{2}^{*}\right)=\frac{1}{\pi} F^{1 / 2}\left(\tilde{z}_{1}\right) F^{1 / 2}\left(\tilde{z}_{2}\right) \int_{-\pi \nu}^{\pi \nu} d u \prod_{c=1}^{M}\left[g_{c}+u\right] e^{-i u\left(\tilde{z}_{1}-\tilde{z}_{2}^{*}\right)}
$$

with $F(\tilde{z})=\sum_{k=1}^{M} \frac{e^{-2 \operatorname{Im} \tilde{z} g_{k}}}{\prod_{s(\neq k)}\left(g_{s}-g_{k}\right)} \theta(\operatorname{Im} \tilde{z})$. Introducing a "characteristic function"

$$
f_{\Gamma}(u)=\sum_{c=1}^{M} \ln \left(1+\frac{u}{g_{c}}\right)
$$

and noticing that

$$
F(\tilde{z})=\theta(\operatorname{Im} \tilde{z}) \sum_{k=1}^{M} \frac{e^{-2 \operatorname{Im} \tilde{z} g_{k}}}{\prod_{s(\neq k)}\left(g_{s}-g_{k}\right)} \equiv\left(\prod_{c} \frac{1}{g_{c}}\right) \frac{1}{4 \pi} \int_{-\infty}^{\infty} d k \exp \left(-i k \operatorname{Im} \tilde{z}-f_{\Gamma}(-i k / 2)\right)(45)
$$

we can rewrite the kernel Eq. (43) in an equivalent form. Denoting $\tilde{z}_{1}=i y_{1}-\omega / 2 ; \tilde{z}_{2}=$ $i y_{2}+\omega / 2$ we find that the kernel is given by :

$$
\begin{array}{rl}
\tilde{K}\left(Z_{1}, Z_{2}^{*}\right)=N^{2} & K\left(\tilde{z}_{1}, \tilde{z}_{2}^{*}\right)=\frac{N^{2}}{4 \pi^{2}} \int_{-\pi \nu}^{\pi \nu} d u e^{-\left(y_{1}+y_{2}\right) u+i \omega u+f_{\Gamma}(u)} \\
& \times\left(\int_{-\infty}^{\infty} d k_{1} \exp \left\{-i k_{1} y_{1}-f_{\Gamma}\left(-i k_{1} / 2\right)\right\} \int_{-\infty}^{\infty} d k_{2} \exp \left\{-i k_{2} y_{2}-f_{\Gamma}\left(-i k_{2} / 2\right)\right\}\right)^{1 / 2}
\end{array}
$$

Using the derived expression we are going to demonstrate that for a system with many open channels $M \gg g$ the statistics of resonances eventually becomes Ginibrelike. This fact coordinates with existing numerical simulations [16, 17, 56.

To this end we consider the case of equivalent channels: $g_{1}=g_{2}=\ldots=g_{M} \equiv g$ and evaluate all the three integrals in Eq.(46) by the saddle-point method in the limit $M \gg g \sim 1$. A straightforward calculation gives

$$
\int_{-\infty}^{\infty} d k_{1} \exp \left\{-i k_{1} y_{p}-f_{\Gamma}\left(-i k_{p} / 2\right)\right\} \approx M^{1 / 2-M} e^{M} \sqrt{2 \pi} \frac{1}{y_{p}}\left(2 g y_{p}\right)^{M} e^{-2 g y_{p}}
$$

for $p=1,2$ whereas the integral over $u$ dominated by the saddle-point $u_{s}=$ $-g+M / y_{s} ; y_{s}=y_{1}+y_{2}-i \omega$ is estimated in the limit $M \rightarrow \infty$ as

$$
M^{M+1 / 2}\left(g y_{s}\right)^{-M} e^{-M+g y_{s}} \sqrt{2 \pi} \frac{1}{y_{s}}
$$

as long as $\left|\operatorname{Re} u_{s}\right| \leq \pi \nu$. In the opposite case $\left|\operatorname{Re} u_{s}\right|>\pi \nu$ the integral is zero. Combining these expressions and taking the absolute value of the kernel results in:

$$
\left|\tilde{K}\left(Z_{1}, Z_{2}^{*}\right)\right|=N^{2} \frac{2^{M-1} M}{\pi} \frac{\left(y_{1} y_{2}\right)^{(M-1) / 2}}{\left[\left(y_{1}+y_{2}\right)^{2}+\omega^{2}\right]^{(M+1) / 2}} \quad ; \quad M \gg g
$$

This expression immediately shows us that the mean density of resonances is

$$
\rho(Z)=\left|\tilde{K}\left(Z, Z^{*}\right)\right|=N^{2} \frac{M}{4 \pi y^{2}}=\frac{M}{4 \pi(\operatorname{Im} Z)^{2}} \quad ; \quad \frac{M}{2(g+\pi \nu)} \leq y \leq \frac{M}{2(g-\pi \nu)}
$$


and zero otherwise. Such a "cloud of resonances with a gap" is in agreement with the earlier result for the mean density obtained by Haake et al.14 in the $\operatorname{limit} M \rightarrow \infty ; N \rightarrow \infty$ in such a way that $M / N=$ fixed.

Let us now suppose that both $Z_{1}$ and $Z_{2}$ are within the "cloud". Then it is possible to rewrite Eq.477) as:

$$
\left|\tilde{K}\left(Z_{1}, Z_{2}^{*}\right)\right|=\left(\rho\left(Z_{1}\right) \rho\left(Z_{2}\right)\right)^{1 / 2}\left(1-\frac{\left(y_{1}-y_{2}\right)^{2}+\omega^{2}}{\left(y_{1}+y_{2}\right)^{2}+\omega^{2}}\right)^{\frac{M+1}{2}}
$$

Taking into account that actually $y_{1,2} \geq M /(2 g+2 \pi \nu) \gg 1$ we see that always $y_{1}+y_{2} \gg \omega \sim 1$ whereas the relation between $y_{1}-y_{2}$ and $\omega$ can be arbitrary. Introducing

$$
\rho\left(\frac{Z_{1}+Z_{2}}{2}\right) \equiv \frac{M N^{2}}{\pi\left(y_{1}+y_{2}\right)^{2}}
$$

we see that

$$
\begin{gathered}
\left|\tilde{K}\left(Z_{1}, Z_{2}^{*}\right)\right|=\left(\rho\left(Z_{1}\right) \rho\left(Z_{2}\right)\right)^{1 / 2}\left(1-\frac{\pi \rho\left(\frac{Z_{1}+Z_{2}}{2}\right)}{M N^{2}}\left[\left(y_{1}-y_{2}\right)^{2}+\omega^{2}\right]\right)^{\frac{M+1}{2}} \\
\rightarrow\left(\rho\left(Z_{1}\right) \rho\left(Z_{2}\right)\right)^{1 / 2} e^{-\frac{1}{2} \pi \rho\left(\frac{Z_{1}+Z_{2}}{2}\right)\left|Z_{1}-Z_{2}\right|^{2}}
\end{gathered}
$$

in the limit $M \rightarrow \infty$. It is further evident, that the correlations vanish very rapidly when the distance $\left|Z_{1}-Z_{2}\right|$ between eigenvalues exceeds $M^{-1 / 2}$. Thus, we can safely put $\rho\left(Z_{1}\right) \approx \rho\left(Z_{2}\right) \approx \rho\left(\frac{Z_{1}+Z_{2}}{2}\right)$ when $M \rightarrow \infty$ arriving finally to the Ginibre-like expression for the kernel:

$$
\left|\tilde{K}\left(Z_{1}, Z_{2}^{*}\right)\right|=\rho(Z) \exp -\frac{1}{2} \pi \rho(Z)\left|Z_{1}-Z_{2}\right|^{2}
$$

In contrast to the Ginibre case, here the density $\rho(Z)$ inside the cloud is not constant but is rather position-dependent. It is natural to conjecture that expression Eq. (49) constitutes the most universal form of the eigenvalue statistics for the case of strongly non-Hermitian random matrices. This conjecture is also supported with existing numerics, e.g. for a QCD-inspired non-Hermitian model [40].

Having at our disposal the correlation functions, we can easily repeat the calculations done in the previous sections and find such statistical characteristics as spectral form-factor and the number variance [61, 57. In particular, for the case of equivalent channels $g_{c}=g, c=1, \ldots, M$ the variance of the number of resonances in a strip $0<\operatorname{Re} Z<L=L_{x} \Delta ;-\infty<\operatorname{Im} Z<\infty$ is given by:

$\Sigma_{2}\left(L_{x}\right)=L_{x}-\frac{1}{\pi^{2}} \int_{0}^{1} \frac{d k}{k^{2}} \sin ^{2}\left(\pi k L_{x}\right) \int_{-(1-k)}^{(1-k)} d v\left[1-\frac{k^{2}}{(g+v)^{2}}\right]^{M}$

where we have used $\pi \nu(0)=1$. Let us discuss for simplicity its typical features for the simplest case of a single open channel $M=1$. We are actually interested in deviations of the number variance from its value known for Hermitian GUE matrices. One finds:

$\delta \Sigma_{2}\left(L_{x}\right)=\Sigma_{2}\left(L_{x}\right)-\Sigma_{2}^{G U E}\left(L_{x}\right)=\frac{2}{\pi^{2}} \int_{0}^{1} d k \sin ^{2}\left(\pi k L_{x}\right) \frac{1-k}{g^{2}-(1-k)^{2}}$

As usual, we are interested in the behaviour of the number variance for $L_{x} \gg 1$, where $\Sigma_{2}^{G U E}\left(L_{x}\right)$ grows logarithmically as: $\Sigma_{2}^{G U E}\left(L_{x}\right) \approx \frac{1}{\pi^{2}} \ln L_{x}+$ const $+\ldots$ For any $g>1$ we find that the difference $\delta \Sigma\left(L_{x}\right)$ tends to a constant value $\frac{1}{2 \pi^{2}} \ln \left[g^{2} /\left(g^{2}-1\right)\right]$. This 
fact means that the curves $\Sigma_{2}^{G U E}\left(L_{x}\right)$ in the asymptotic regime $L_{x} \gg 1$ are just shifted upwards by a constant amount. The shift is the larger the more "open" is the system, i.e. the closer is the value of $g$ to unity. The so-called "perfect coupling" case $g=1$ is known to be in many respects specific [11. Physically, it describes the situation when direct scattering is completely absent. On a level of number variance such a specificity is reflected in changing the coefficient in front of the leading logarithmic growth from the GUE value $1 / \pi^{2}$ to a new value $3 /\left(2 \pi^{2}\right)$.

Qualitatively, the same picture holds for $M>1$ : for a fixed value of the strip widths $L_{x}$ the number variance is the larger, the larger is $M$ and the closer are the coupling constants $g$ to unity. This fact is just a fingerprint of a gradual "decorrelation" of resonance positions along the real axis. As to the small-distance behaviour of the nearest-neighbour distance distribution $p\left(Z_{0}, S \ll \Delta\right)$ in the complex plane, the leading term for $S \rightarrow 0$ turns out to be always cubic: $p\left(Z_{0}, S \ll \Delta\right) \propto S^{3}$, as long as the system is open, in agreement with the existing numerical data [17, 18, 16]. However, for few open channels and $g \gg 1$ the interval of cubic behaviour turns out to be parametrically small: $S \ll g^{-1} \Delta$. For the observation points $Z_{0}$ taken at regions with asymptotically low density of resonances a crossover to an anomalous $S^{5 / 2}$ behaviour might be observable, cf. [50].

In the end of the present section we discuss briefly an intimate relationship which actually exists between the kernel Eq. (43) derived for a finite-rank perturbation and its Gaussian counterpart, Eq.(17). For this we notice, that the kernel Eq.(43) derived under an assumption of positivity of each $\gamma_{c}$ ( hence $g_{c}$ ), but in fact its form Eq. (46) describes the statistics of complex eigenvalues for arbitrary finite-rank perturbations, irrespective of the sign of a particular eigenvalue $\gamma_{c}$. This fact can be proven by a straightforward modification of the technique outlined in the present section. Moreover, a little thought makes it natural to expect that its validity is not restricted even by finite-rank perturbations, but rather comprises the whole class of almost-Hermitian matrices, characterized by $N \operatorname{Tr} \Gamma^{2} \propto \operatorname{tr} H^{2}$ in the limit $N \rightarrow \infty$. As a confirmation of this conjecture, let us look from this point of view on a typical Gaussian deformation. For that case a typical eigenvalue of $\Gamma$ is $\gamma_{c} \sim N^{-1 / 2} \ll 1$ and therefore $g_{c}^{-1} \approx 2 \gamma_{c}, c=1, \ldots, N$. This fact allows us to expand the "characteristic function" in Eq. (44) as:

$$
\left.f_{\Gamma}(u)\right|_{N \rightarrow \infty} \approx 2 u \operatorname{tr} \Gamma-2 u^{2} \operatorname{Tr} \Gamma^{2}+\ldots
$$

It is an easy task to show that for a Gaussian $\Gamma$ we have typically $\operatorname{Tr} \Gamma \sim N^{-1 / 2} \ll$ $\operatorname{Tr} \Gamma^{2} \sim O(1)$. Therefore one can put safely: $f_{\Gamma}(u) \approx-2 u^{2} \operatorname{Tr} \Gamma^{2}$ which renders the $k$-integrals in Eq. (46) to be Gaussian ones. Identifying: $\operatorname{Tr} \Gamma^{2}=\alpha^{2} / 4$ and performing the Gaussian integrals we see that the resulting kernel coincides with that given in Eq. (17), up to an overall phase factor $e^{i x / 2\left(y_{2}-y_{1}\right)}$ which in any way does not play any role in calculating the correlation functions of eigenvalues.

\subsection{Density of complex eigenvalues via supersymmetry approach}

Unfortunately, it is not known at the moment how to extend the methods described above to the case of non-Hermitian deformations of real symmetric matrices. We consider this issue as one of the most challenging problems for future investigations. At the same time the mean density of complex eigenvalues turns out to be accessible via a technique known as Efetov supersymmetry approach and is shortly discussed below. In fact, the method provides access to non-Hermitian deformations of matrices interpolating between real symmetric and complex Hermitian, as discussed in [50]. 
As before we decompose any $N \times N$ matrix $\hat{J}$ into a sum of its Hermitian and skew-Hermitian parts and consider an ensemble of random $N \times N$ complex matrices $\hat{J}=\hat{H}_{1}+i v \hat{H}_{2}$ where $\hat{H}_{p} ; p=1,2$ are both Hermitian: $\hat{H}_{p}^{\dagger}=\hat{H}_{p}$. The parameter $v$ is used to control the degree of non-Hermiticity. In turn, complex Hermitian matrices $\hat{H}_{p}$ can always be represented as $\hat{H}_{1}=\hat{S}_{1}+i u \hat{A}_{1}$ and $\hat{H}_{2}=\hat{S}_{2}+i w \hat{A}_{2}$, where $\hat{S}_{p}=\hat{S}_{p}^{T}$ is a real symmetric matrix, and $\hat{A}_{p}=-\hat{A}_{p}^{T}$ is a real antisymmetric one. From this point of view the parameters $u, w$ control the degree of being non-symmetric.

In the first part of this subsection we consider the matrices $\hat{S}_{1}, \hat{S}_{2}, \hat{A}_{1}, \hat{A}_{2}$ to be mutually statistically independent, with i.i.d. entries normalized in such a way that

$$
\lim _{N \rightarrow \infty} \frac{1}{N} \operatorname{Tr} \hat{S}_{p}^{2}=\lim _{N \rightarrow \infty} \frac{1}{N} \operatorname{Tr} \hat{A}_{p} \hat{A}_{p}^{T}=1
$$

Such a normalization ensures that for any fixed value of the parameter $u>0$ statistics of real eigenvalues of the Hermitian matrix $\hat{H}=\hat{S}+i u \hat{A}$ in the limit $N \rightarrow \infty$ is identical (up to a trivial rescaling) to that of $u=1$, the latter case being standard GUE. On the other hand, for $u \equiv 0$ real eigenvalues of the real symmetric matrix $\hat{S}$ follow a different pattern of the Gaussian Orthogonal Ensemble (GOE). The nontrivial crossover between GUE and GOE types of statistical behaviour happens on a scale $u \propto N^{-1 / 2}[1]$.

Similar arguments show [47, 50], that the most interesting behaviour of complex eigenvalues of non-Hermitian matrices should be expected for the parameter $v$ being scaled in a similar way: $v \propto N^{-1 / 2}$. It is just the regime of weak non-Hermiticity which we are interested in. Under these conditions a non-Hermitian matrix $\hat{J}$ still "remembers" the statistics of its Hermitian part $\hat{H}_{1}$. As will be clear afterwards, the parameter $w$ should be kept of the order of unity in order to influence the statistics of the complex eigenvalues.

Correspondingly, we scale the parameters as $\boldsymbol{\Upsilon}$ :

$$
v=\frac{\alpha}{2 \sqrt{N}} ; \quad u=\frac{\phi}{2 \sqrt{N}}
$$

and consider $\alpha, \phi, w$ fixed of the order $\mathrm{O}(1)$ when $N \rightarrow \infty$.

For calculating the mean density of complex eigenvalues $Z_{k}=X_{k}+i Y_{k}, \quad k=$ $1,2, \ldots, N$ defined as

$$
\rho(Z)=\sum_{k=1}^{N} \delta^{(2)}\left(Z-Z_{k}\right)=\sum_{k=1}^{N} \delta\left(X-X_{k}\right) \delta\left(Y-Y_{k}\right) \equiv \rho(X, Y)
$$

one relates it to the "charge potential" [70]:

$$
\Phi(X, Y, \kappa)=\overline{\frac{1}{2 \pi} \ln \operatorname{Det}\left[\left(Z-\mathcal{H}_{e f f}\right)\left(Z-\mathcal{H}_{e f f}\right)^{\dagger}+\kappa^{2}\right]}
$$

in view of the relation: $\rho(X, Y)=\lim _{\kappa \rightarrow 0} \partial^{2} \Phi(X, Y, \kappa)$, where $\partial^{2}$ stands for the two-dimensional Laplacian, see appendix A of [11]. Technically, it is convenient to introduce the generating function (cf.[11])

$$
\mathcal{Z}=\frac{\operatorname{Det}\left[\left(Z-\mathcal{H}_{e f f}\right)\left(Z-\mathcal{H}_{e f f}\right)^{\dagger}+\kappa^{2}\right]}{\operatorname{Det}\left[\left(Z_{b}-\mathcal{H}_{e f f}\right)\left(Z_{b}-\mathcal{H}_{e f f}\right)^{\dagger}+\kappa^{2}\right]}
$$

in terms of which

$$
\rho(Z)=-\frac{1}{\pi} \lim _{\kappa \rightarrow 0} \frac{\partial}{\partial Z^{*}} \lim _{Z_{b} \rightarrow Z} \frac{\partial}{\partial Z_{b}} \mathcal{Z}
$$

ศ In the Letter [50] there is a misprint in the definition of the parameter $\alpha$. 
Assuming the matrix elements of all involved matrices to be statistically independent, up to symmetry constraints, one performs the ensemble averaging by the standard trick of representing the ratio of the two determinants in Eq.(156) in terms of Gaussian superintegrals. After a set of manipulations exposed in [50] one arrives at the following expression:

$\langle\rho(X, y)\rangle=\frac{N \nu(X)}{16} \int d \mu(\hat{Q}) \operatorname{Str}\left(\hat{\sigma}_{\tau}^{(F)} \hat{Q}\right) \operatorname{Str}\left(\hat{\sigma}_{\tau} \hat{Q}\right) \exp -S(\hat{Q})$

$S(\hat{Q})=-\frac{i}{2} y \operatorname{Str}\left(\hat{\sigma}_{\tau} \hat{Q}\right)-\frac{a^{2}}{16} \operatorname{Str}\left(\hat{\sigma}_{\tau} \hat{Q}\right)^{2}+\frac{b^{2}}{16} \operatorname{Str}\left(\hat{\tau}_{2} \hat{Q}\right)^{2}-\frac{c^{2}}{16} \operatorname{Str}(\hat{\sigma} \hat{Q})^{2}$

where we introduced the scaled imaginary parts $y=\pi \nu(X) N Y$, with $\nu(X)$ being the mean (semicircular) eigenvalue density. We also used the notations: $a^{2}=$ $(\pi \nu(X) \alpha)^{2}, \quad b^{2}=(\pi \nu(X) \phi)^{2}, \quad c^{2}=(\pi \nu(X) \alpha w)^{2}$. Here the integration goes over the set of $8 \times 8$ supermatrices $\hat{Q}$ satisfying the constraint $\hat{Q}^{2}=-1$. The symbols Str, Sdet stand here for the graded trace and the graded determinant, correspondingly.

Properties of these matrices and the integration measure $d \mu(\hat{Q})$ can be found in [29]. The matrices $\hat{\sigma}_{\tau}^{(F)}, \hat{\sigma}_{\tau}$ and $\hat{\tau}_{2}$ in Eq. (58) are some fixed block-diagonal $8 \times 8$ supermatrices, and can be found in 50. The whole expression is just a general $\sigma-$ model representation of the mean density of complex eigenvalues in the regime of weak non-Hermiticity. We expect it to be universal and applicable even beyond the case of matrices with independent entries, after replacement of the semicircular density $\nu(X)$ with actual density of real eigenvalues of the Hermitian part. The expression is parametrised by $a, b$ and $c$. The parameters controll the degree of non-Hermiticity $(a)$, and symmetry properties of the Hermitian (b) and non-Hermitian (c) parts of the ensemble.

Still, in order to obtain an explicit expression for the density of complex eigenvalues one has to evaluate the integral over the set of supermatrices $\hat{Q}$. In general, it is an elaborate task due to the complexity of that manifold. At the present moment such an evaluation was successfully performed for three "pure" cases: those of almostHermitian matrices [47, real almost-symmetric matrices 45] and complex symmetric matrices [52. The first case (which is technically the simplest one) corresponds to $\phi \rightarrow \infty$, that is $b \rightarrow \infty$. Under this condition only that part of the matrix $\hat{Q}$ which commutes with $\hat{\tau}_{2}$ provides a nonvanishing contribution and the resulting integral 47] proves to be equivalent to the "diagonal" part $K\left(Z, Z^{*}\right)$ of the kernel Eq.(17), as expected.

The second nontrivial case for which the result is known explicitly is due to Efetov [45]. This is the case of real matrices with "weak asymmetry". In the present notations this case corresponds to the limit $\phi \rightarrow 0 ; w \rightarrow \infty$ in such a way that the product $\phi w=\tilde{c}$ is kept fixed. The density of complex eigenvalues turns out to be given by:

$$
\begin{aligned}
\rho_{X}(y)=\delta(y) \int_{0}^{1} d t \exp \left(-\tilde{c}^{2} t^{2} / 2\right) \\
\quad+2 \sqrt{\frac{2}{\pi}} \frac{|y|}{\tilde{c}} \int_{1}^{\infty} d u \exp \left(-\frac{2 y^{2} u^{2}}{\tilde{c}^{2}}\right) \int_{0}^{1} d t t \sinh (2 t|y|) \exp \left(-\tilde{c}^{2} t^{2} / 2\right)
\end{aligned}
$$

The first term in this expression shows that everywhere in the regime of "weak asymmetry" $\tilde{c}<\infty$ a finite fraction of eigenvalues remains on the real axis. Such a behaviour is qualitatively different from that typical for the case of "weak nonHermiticity" $\tilde{a}<\infty$, where eigenvalues acquire a nonzero imaginary part with 
probability one. In the limit $\tilde{c}>>1$ the portion of real eigenvalues behaves like $\tilde{c}^{-1}$. Remembering the normalization of the parameter $v$, Eq. (153), it is easy to see that for the case of $v=O(1)$ the number of real eigenvalues should scale as $\sqrt{N}$. The fact that of the order of $N^{1 / 2}$ eigenvalues of strongly asymmetric real matrices stay real was first found numerically by Sommers et al. [70, 79, and proven by Edelman [65.

At last, the case of complex symmetric matrices $(b=c=0$ in the present notation) turns out to be most involved technically. The result of evaluating the integral Eq. (58) over the coset space [52] turns out to be:

$\left\langle\rho_{X}(y)\right\rangle=-\frac{1}{16 \pi} \frac{\partial}{\partial y} \int_{-1}^{1} d \lambda F^{-2}(\lambda) \int_{-\infty}^{\infty} d \lambda_{1} F\left(i \lambda_{1}\right) \int_{\lambda_{1}}^{\infty} d \lambda_{2} F\left(i \lambda_{2}\right) \frac{\left(\lambda_{2}-\lambda_{1}\right)\left(2 \lambda-i \lambda_{1}-i \lambda_{2}\right)}{\left(\lambda-i \lambda_{1}\right)^{2}\left(\lambda-i \lambda_{2}\right)}(61)$

where

$$
F(\lambda)=e^{\lambda y+a^{2} \lambda^{2}} \frac{1}{\left(1-\lambda^{2}\right)^{1 / 2}}
$$

Actually, the integrals over $\lambda_{1}, \lambda_{2}$ as they stand in Eq. (61) should be understood in the sense of a principal value. To be precise, the correct expression is equal to the half-sum of two integrals with integration contours encircling the singular point $\lambda_{1}=-i \lambda ; \lambda_{2}=-i \lambda$ from above and from below.

Of course, the supersymmetry approach is not restricted to anti-Hermitian deformations $i \hat{\Gamma}$ whose entries are statistically independent as assumed above, but can be used as well for fixed finite-rank deformations. This allows one to calculate the density of resonance poles in the complex energy plane. In fact, such a calculation was the first application of the supersymmetry approach to non-Hermitian problems [15] and paved the way to all later developments in [47, 45, 39, 52.

One finds the following $\sigma$-model representation for the distribution of scaled resonance widths $y=\frac{\pi \operatorname{Im} Z}{\Delta}<0$ (measured in units of the local mean level spacing $\Delta$ of the closed system) for the resonances whose positions are within a narrow window around the central point $X=0$ of the spectrum.

$$
\begin{gathered}
\left\langle\rho_{X}(y)\right\rangle=\frac{1}{16} \int d \mu(\hat{Q}) \operatorname{Str}\left(\hat{\sigma}_{\tau}^{(F)} \hat{Q}\right) \operatorname{Str}\left(\hat{\sigma}_{\tau} \hat{Q}\right) \exp \frac{i}{2} y \operatorname{Str}\left(\hat{\sigma}_{\tau} \hat{Q}\right) \\
\times \prod_{a=1}^{M} \operatorname{Sdet}^{-1 / 4}\left[1-\frac{i}{2 g_{a}}\left\{\hat{Q}, \hat{\sigma}_{\tau}\right\}\right] .
\end{gathered}
$$

Here $\left\{\hat{Q}, \hat{\sigma}_{\tau}\right\}=\hat{Q} \hat{\sigma}_{\tau}+\hat{\sigma}_{\tau} \hat{Q}$ stands for the anticommutator.

Performing the integration over the manifold of the supermatrices $\hat{Q}$ (which is of different form for different symmetry classes) one finds for the case of broken invariance [15] the distribution coinciding with that following from the kernel Eq. (43), whereas for the case of preserved invariance the distribution is given by Eq. (61), but with the function $F(\lambda)$ replaced with the following expression [52]:

$$
F_{\Gamma}(\lambda)=\frac{1}{\prod_{c=1}^{M}\left(g_{c}-\lambda\right)^{1 / 2}} \frac{e^{\lambda y}}{\left(1-\lambda^{2}\right)^{1 / 2}}
$$

Such a replacement looks completely natural from the point of view of the correspondence pointed out in the end of preceding section, see Eq. (52).

Finally, we would like to mention a finite-rank generalization of Efetov's result, Eq.601) as performed in 61. Namely, we consider the ensemble of real asymmetric 
matrices of the form: $H+A$, with $H$ being a real symmetric (taken from GOE) and $A$ being a fixed real antisymmetric: $A_{i j}=-A_{j i}$ of a finite rank $M$. As is well-known, a general antisymmetric matrix $A$ of even dimension can be brought by an orthogonal rotation to the following block-diagonal form: $\operatorname{diag}\left(A_{1}, \ldots, A_{N}\right)$, with each block $A_{i}$ being $2 \times 2$ matrix of the form $A_{i}=\left(\begin{array}{cc}0 & \mu_{i} \\ -\mu_{i} & 0\end{array}\right)$. Invoking the argument of the rotational invariance of GOE matrices, it is enough to consider deformations to be of such block-diagonal form. We restrict our attention to the finite-rank case, when all $\mu_{i}=0$ for $i>M$. Introducing the scaled variable $y=\pi \nu(X) N \operatorname{Im} Z$ we find for the rescaled density $\rho_{X}(y)=\langle\rho(Z)\rangle[N \pi \nu(X)]^{-2}$ the following expression [61]:

$$
\begin{aligned}
\rho_{X}(y)=\delta(y) \int_{0}^{1} d t e^{\frac{1}{2}\left[f_{A}(\pi \nu t)+f_{A}(-\pi \nu t)\right]}+\frac{1}{2 \pi} \int_{0}^{1} d t t \sinh (|y| t) e^{\frac{1}{2}\left[f_{A}(\pi \nu t)+f_{A}(-\pi \nu t)\right]} \\
\times \int_{|y|}^{\infty} d s \int_{-\infty}^{\infty} d k \exp \left\{-i k s-\frac{1}{2}\left[f_{A}(i \pi \nu k)+f_{A}(-i \pi \nu k)\right]\right\}
\end{aligned}
$$

where the function $f_{A}(z)$ is given by the same expression as Eq. (44), but with $\gamma_{c}$ replaced by $\mu_{i}$, i.e. $g_{c}$ replaced by $\left(\mu_{i}+1 / \mu_{i}\right) / 2$.

Our interest in the problem was stimulated by the papers 39] claiming that expression Eq. 60), derived for Gaussian A, describes well the complex eigenvalue density even for non-random antisymmetric deformations by matrices $A=\left(\begin{array}{cc}0 & \mu \hat{\mathbf{1}} \\ -\mu \hat{\mathbf{1}} & 0\end{array}\right)$, with a constant $\mu$ being of the order of $\mu \sim N^{-1 / 2}$. This fact immediately follows from Eq. Indeed, for $M \propto N$ and the typical $\mu_{i}$ of the order of $N^{-1 / 2}$ the function $f_{A}(v)$ can be expanded up to a first non-vanishing order such that $f_{A}(v)+f_{A}(-v) \propto v^{2} \operatorname{Tr} A^{2}$, cf. Eq. (152). The corresponding expression then indeed coincides with Eq. (60).

\section{Eigenvalue Statistics of Subunitary Matrices}

\subsection{Truncations of random unitary matrices}

Let us first consider truncated matrices drawn from the circular unitary ensemble (CUE). Let $U=\left(\begin{array}{cc}A & B \\ C & D\end{array}\right)$ be an $N \times N$ matrix from CUE and $A$ a subunitary $(N-M) \times(N-M)$ matrix. To make contact to the general case discussed in the introduction we note that putting $B=0$ and $D=0$ corresponds to a matrix $\hat{A}=\hat{u} \sqrt{\mathbf{1 - \hat { T }}}$ with $T_{i}=0$ for $i=1, \ldots, N-M$ and $T_{i}=1$ for $i=N-M+1, \ldots, N$. This matrix has the same nonzero eigenvalues as the subblock $A$ of the matrix $U$ and we determine below the joint density of those eigenvalues inside the complex unit circle.

The joint density of elements of $U$ can be written as

$P(U) \propto \delta\left(A^{\dagger} A+C^{\dagger} C-\mathbf{1}\right) \delta\left(A^{\dagger} B+C^{\dagger} D\right) \delta\left(B^{\dagger} B+D^{\dagger} D-\mathbf{1}\right)$

with appropriate matrix $\delta$-functions. Integrating out $B$ and $D$ we obtain as joint density of elements of $A$

$$
P(A) \propto \int d C \delta\left(A^{\dagger} A+C^{\dagger} C-\mathbf{1}\right)
$$

with a $2 M(N-M)$ dimensional integration over the complex parameters $C$. Performing these remaining integrations is possible after representing the $\delta$-function 
in Eq. (67) as a Fourier transform over the set of Hermitian matrices $\mathcal{F}$ of the size $(N-M) \times(N-M)$. Then integrals over $C$ are essentially Gaussian and can be immediately performed yielding:

$$
P(A) \propto \int d \mathcal{F} \operatorname{det} \mathcal{F}^{-M} \exp \left\{i \operatorname{Tr} \mathcal{F}\left(\mathbf{1}-A^{\dagger} A\right)\right\}
$$

The matrix integrals of this type emerge frequently in various context, and most recently were calculated in 91 92. For the case $M \geq N / 2$ the probability $P(A)$ is given by a simple, non-singular expression:

$$
P(A) \propto \operatorname{det}\left\{\mathbf{1}-A^{\dagger} A\right\}^{2 M-N} \theta\left\{\mathbf{1}-A^{\dagger} A\right\}
$$

where the matrix $\theta$-function is unity when its argument is a positive definite matrix and is zero otherwise.

In the opposite, most interesting case $M<N / 2$ the integral still can be calculated explicitly [92] but the result is complicated and contains a product of many singular $(\delta$-functional) contributions.

We are however mostly interested in finding the joint probability density of complex eigenvalues rather than just distribution of the matrix $A$ itself. This can be achieved directly from the equation Eq.676) if we again use the Schur decomposition $A=V(z+\hat{R}) V^{-1}$ extensively exploited by us in Sec.2. Here the transformation $V$ is unitary, $z$ is a diagonal matrix of the complex eigenvalues of $A$ and $\hat{R}$ is strictly upper triangular. A procedure allowing one to integrate out matrices $V$, the $(N-M)(N-M-1) / 2$ complex parameters $R_{i j}$ and the $M(N-M)$ complex parameters $C$ is described in some detail in [36. The resulting expression is however rather simple:

$$
P(\{z\}) \propto \prod_{i<j}^{1 \ldots N-M}\left|z_{i}-z_{j}\right|^{2} \prod_{i=1}^{N-M}\left(1-\left|z_{i}\right|^{2}\right)^{M-1} \theta\left(1-\left|z_{i}\right|^{2}\right)
$$

This distribution is very analogous to the Ginibre ensemble and all correlation functions can be found readily by the method of orthogonal polynomials. In fact, the powers $z^{n-1}$ are already orthogonal inside the unit circle of the complex plane. An equivalent method is to consider the joint density $P(\{z\})$ as the absolute square of a Slater determinant of normalized wave functions

$$
\phi_{n}(z)=z^{n-1} w\left(|z|^{2}\right) / \sqrt{N_{n}}
$$

, with $N_{n}$ standing for a normalization factor and the domain of integration being $|z| \leq 1$. The kernel, which determines all correlation functions is given by

$$
K\left(z_{1}, z_{2}^{*}\right)=\sum_{n=1}^{N-M}\left(z_{1} z_{2}^{*}\right)^{n-1} w\left(\left|z_{1}\right|^{2}\right) w\left(\left|z_{2}\right|^{2}\right) / N_{n} .
$$

Further defining $x=r^{2}=|z|^{2}$ we can, for example, easily write down the averaged density of complex eigenvalues at point $|z|$ as

$$
P(r)=\frac{2 r}{N-M} \frac{(1-x)^{M-1}}{(M-1) !}\left(\frac{d}{d x}\right)^{M} \frac{\left(1-x^{N}\right)}{1-x} .
$$

There are two important limiting cases for large $N$ : either $\mu=M / N$ fixed or $M$ fixed. For fixed $\mu$ and $N \rightarrow \infty$ we find the scaling behaviour:

$$
P(r)=\frac{\mu}{1-\mu} \frac{2 r}{\left(1-r^{2}\right)^{2}}
$$


for $r^{2}<1-\mu$ and $P(r)=0$ otherwise. The distribution shows a gap near the unit circle and qualitatively resembles one obtained for resonances in the chaotic scattering problem for large number of channels, see Haake et al.14. In this "strongly nonunitary" - limit one can also simplify the cluster function given by $Y\left(z_{1}, z_{2}\right)=\left|K\left(z_{1}, z_{2}^{*}\right)\right|^{2}$ :

$$
Y(z, z+\delta)=(N-M)^{2} \rho(z)^{2} \exp \left(-\pi(N-M) \rho(z)|\delta|^{2}\right)
$$

This is just the Ginibre behaviour Eq. (49) with the distance $\delta$ rescaled by the local mean level distance $1 / \sqrt{(N-M) \rho(z)}$ given by (72) through $\rho(z)=P(r) / 2 \pi r$. The same can be shown for the nearest neighbour distance distribution obtained by Grobe et al. [80] and applied to a damped chaotic kicked top.

In the other limit of fixed $M$ and $N$ to $\infty$ the matrices may be considered as weakly nonunitary, and we recover exactly the universal resonance-width distribution [15] 11] for perfect coupling to $M$ channels with $y=N(1-r)$

$$
\rho(y)=\frac{y^{M-1}}{(M-1) !}\left(\frac{-d}{d y}\right)^{M} \frac{1-e^{-2 y}}{2 y} .
$$

Similarly the cluster function obtained in this limit can be shown to coincide with the one obtained in the previous section for chaotic scattering with a finite number $M$ of perfectly coupled channels. The statistics (74) has also been found by Kottos and Smilansky 22] for chaotic scattering on graphs and by Glück et al.23] for a model of crystal electron in the presence of dc and ac fields. In both of these works the $S$-matrix is reduced to the resolvent of a subunitary matrix as is investigated in the present subsection.

\subsection{General finite-rank deviations from unitarity}

In what follows we assume all eigenvalues of the deviation matrix $\hat{T}=1-\hat{G}$ are smaller than unity: $T_{i}<1$, but the resulting expressions turn out to be valid in the limiting cases some $T_{i}=1$ as well.

We again start with the Schur decomposition $\hat{A}=\hat{U}(\hat{Z}+\hat{R}) \hat{U}^{\dagger}$ of the matrix $\hat{A}$ in terms of a unitary matrix $\hat{U}$, a diagonal matrix of the eigenvalues $\hat{Z}=\{z\}$ and a upper triangular matrix $\hat{R}$. One can satisfy oneself, that the eigenvalues $z_{1}, \ldots, z_{N}$ are generically not degenerate, provided all $T_{i}<1$. Then, the measure written in terms of new variables is given by $d \hat{A}=|\Delta(\{z\})|^{2} d \hat{R} d \hat{Z} d \mu(\hat{U})$, where the first factor is just the squared Vandermonde determinant of eigenvalues $z_{i}$ and $d \mu(\hat{U})$ is the invariant measure on the unitary group. The joint probability density of complex eigenvalues is then given by:

$\mathcal{P}(\{z\}) \propto|\Delta(\{z\})|^{2} \int d \mu(\hat{U}) d \hat{R} \delta\left((\hat{Z}+\hat{R})(\hat{Z}+\hat{R})^{\dagger}-\hat{U}^{\dagger} \hat{G} \hat{U}\right)$.

The integration over $\hat{R}$ can again be performed using its triangularity (similar to the case of "truncated" matrices considered above). We give here a derivation in more detail. Let us consider the columns of the $N \times N$ unitary matrices $U$ as $N$-component (mutually orthogonal) vectors $\mathbf{a}_{l}, l=1, \ldots, N$, so that $\hat{U}=\left(\mathbf{a}_{1}, \mathbf{a}_{2}, \ldots, \mathbf{a}_{N}\right)$. Then the $\delta$-functions in Eq.(75) imply

$$
\left|z_{i}\right|^{2}+\sum_{k(<i)}\left|R_{k i}\right|^{2}+\mathbf{a}_{i}^{\dagger} \hat{T} \mathbf{a}_{i}=1
$$


and for $i<j$ :

$$
z_{i}^{*} R_{i j}+\sum_{k(<i)} R_{k i}^{*} R_{k j}+\mathbf{a}_{i}^{\dagger} \hat{T} \mathbf{a}_{j}=0
$$

Integration over the complex parameters $R_{i j}$ can easily be done and yields the Jacobian $1 / \prod_{i<j}\left|z_{i}\right|^{2}$. The remaining integration over the unitary group should be done with the product of the diagonal $\delta$-functions as integrand. There the solution $R_{i j}$ of Eq. (777) has to be inserted. We can find that solution employing the hierarchical structure of the equations. It can easily be checked, that the solution is given by

$$
R_{k i}=-\frac{1}{z_{k}^{*}} \mathbf{a}_{k}^{\dagger} \frac{1}{1-\hat{T}\left(\mathbf{a}_{1} \otimes \mathbf{a}_{1}^{\dagger}+\ldots+\mathbf{a}_{k-1} \otimes \mathbf{a}_{k-1}^{\dagger}\right)} \hat{T} \mathbf{a}_{i} .
$$

One may also show that Eq.(177) can be extended to $i=j$ such that

$$
\begin{aligned}
& -z_{i}^{*} R_{i i}=\sum_{k<i}\left|R_{k i}\right|^{2}+\mathbf{a}_{i}^{\dagger} \hat{T} \mathbf{a}_{i} \\
& =\mathbf{a}_{i}^{\dagger} \frac{1}{1-\hat{T}\left(\mathbf{a}_{1} \otimes \mathbf{a}_{1}^{\dagger}+\ldots+\mathbf{a}_{i-1} \otimes \mathbf{a}_{i-1}^{\dagger}\right)} \hat{T} \mathbf{a}_{i} .
\end{aligned}
$$

This implies

$$
\begin{aligned}
\mathcal{P}(\{z\}) \propto|\Delta(\{z\})|^{2} & \frac{1}{\prod_{i<j}\left|z_{i}\right|^{2}} \\
& \times \int d \mu(\hat{U}) \prod_{i} \delta\left(\left|z_{i}\right|^{2}-1+\mathbf{a}_{i}^{\dagger} \frac{1}{1-\hat{T}\left(\mathbf{a}_{1} \otimes \mathbf{a}_{1}^{\dagger}+\ldots+\mathbf{a}_{i-1} \otimes \mathbf{a}_{i-1}^{\dagger}\right)} \hat{T} \mathbf{a}_{i}\right) .
\end{aligned}
$$

Finally we use the identity

$$
1-\mathbf{a}_{i}^{\dagger} \frac{1}{1-\hat{T}\left(\mathbf{a}_{1} \otimes \mathbf{a}_{1}^{\dagger}+\ldots+\mathbf{a}_{i-1} \otimes \mathbf{a}_{i-1}^{\dagger}\right)} \hat{T} \mathbf{a}_{i}=\frac{\operatorname{det}\left(1-\hat{T}\left(\mathbf{a}_{1} \otimes \mathbf{a}_{1}^{\dagger}+\ldots+\mathbf{a}_{i} \otimes \mathbf{a}_{i}^{\dagger}\right)\right)}{\operatorname{det}\left(1-\hat{T}\left(\mathbf{a}_{1} \otimes \mathbf{a}_{1}^{\dagger}+\ldots+\mathbf{a}_{i-1} \otimes \mathbf{a}_{i-1}^{\dagger}\right)\right)}
$$

and introducing the projector matrices $\hat{P}_{l}=\operatorname{diag}(1, \ldots 1,0, \ldots, 0)$ after a simple algebra we arrive at the following expression:

$$
\mathcal{P}(\{z\}) \propto|\Delta(\{z\})|^{2} \int d \mu(\hat{U}) \prod_{l=1}^{N} \delta\left(\left|z_{1}\right|^{2} \ldots\left|z_{l}\right|^{2}-\operatorname{det}\left[\mathbf{1}-\hat{T} \hat{U} \hat{P}_{l} \hat{U}^{\dagger}\right]\right) .
$$

The remaining integration over the unitary group can be done, in essence, similar to the corresponding procedure for non-Hermitian matrices, see Section 2.2. Actual calculations are, however, more involved and we outline the main steps of the full solution below extending 63 .

First, introduce $N \times N$ matrices of $\operatorname{rank} l: \hat{Q}_{l}=\sum_{i=1}^{l} \mathbf{a}_{i} \otimes \mathbf{a}_{i}^{\dagger}$, so that $\hat{U} \hat{P}_{l} \hat{U}^{\dagger}=\hat{Q}_{l}$. Due to the fact that only $M$ out of $N$ eigenvalues of the matrix $\hat{T}$ are non-zero, both the matrices $\hat{Q}_{l}$ and the vectors a can be effectively taken to be of the size $M$ (it amounts to changing the unspecified normalization constant in Eq.811). We then redefine the matrix $\hat{T}$ as $\hat{T}=\operatorname{diag}\left(T_{1}, \ldots, T_{M}\right)=\hat{\tau}^{\dagger} \hat{\tau}$, i.e. the matrix of transmission coefficients $0 \leq T_{a} \leq 1$.

Writing down the corresponding constraints in a form of $\delta$ - functions, one can represent the expression Eq. (75) in the form:

$$
\mathcal{P}(\{z\}) \propto|\Delta(\{z\})|^{2} \int \prod_{i=1}^{N} d^{2} \mathbf{a}_{\mathbf{i}} \delta\left(\mathbf{1}-\sum_{j=1}^{N} \mathbf{a}_{\mathbf{j}} \otimes \mathbf{a}_{\mathbf{j}}^{\dagger}\right)
$$




$$
\times \prod_{l=1}^{N} \delta\left(\left|z_{1}\right|^{2} \ldots\left|z_{l}\right|^{2}-\operatorname{det}\left[\mathbf{1}-\hat{T} \sum_{j=1}^{l} \mathbf{a}_{\mathbf{j}} \otimes \mathbf{a}_{\mathbf{j}}^{\dagger}\right]\right) .
$$

Here $\mathbf{a}_{\mathbf{i}}, \quad i=1, \ldots, N$ are $M$-dimensional complex vectors building a $N \times M$ subblock of a unitary matrix $U$ and we define $d^{2} \mathbf{a}_{\mathbf{i}}:=\prod_{j=1}^{M} \operatorname{Re} d \mathbf{a}_{\mathbf{i}}{ }^{(j)} \operatorname{Im} d \mathbf{a}_{\mathbf{i}}{ }^{(j)}$. The unitary constraint is forced by the inserted $\delta$-function. Obviously $\left|z_{i}\right|^{2}=$ $\operatorname{det}\left(\mathbf{1}-\hat{T} \sum_{j=1}^{i} \mathbf{a}_{\mathbf{j}} \otimes \mathbf{a}_{\mathbf{j}}^{\dagger}\right) / \operatorname{det}\left(\mathbf{1}-\hat{T} \sum_{j=1}^{i-1} \mathbf{a}_{\mathbf{j}} \otimes \mathbf{a}_{\mathbf{j}}^{\dagger}\right) \leq 1$ as it should be for complex eigenvalues of a contraction.

We want to integrate over the unitary group which amounts to integrate over all complex vectors $\mathbf{a}_{\mathbf{i}}$. We increase again the number of integration variables introducing as independent variables matrices $q_{i}$ through the dyadic product $q_{j}=\mathbf{a}_{\mathbf{j}} \otimes \mathbf{a}_{\mathbf{j}}^{\dagger}$. The $M \times M$ Hermitian matrix $q_{i}$ contains in general more independent real variables than the complex vector $\mathbf{a}_{\mathbf{i}}$. Therefore we insert the identities $1=\int d q_{i} \delta\left(q_{i}-\mathbf{a}_{\mathbf{i}} \otimes \mathbf{a}_{\mathbf{i}}^{\dagger}\right)$ with integrations over the Hermitian matrices $q_{i}$ and the corresponding $\delta$-functions for independent elements. Then (82) takes the form

$$
\begin{aligned}
\mathcal{P}(\{z\}) & \propto|\Delta(\{z\})|^{2} \int \prod_{i=1}^{N} d q_{i} \delta\left(\mathbf{1}-\sum_{j=1}^{N} q_{j}\right) \prod_{i=1}^{N} \int d^{2} \mathbf{a} \delta\left(q_{i}-\mathbf{a} \otimes \mathbf{a}^{\dagger}\right) \\
& \times \prod_{l=1}^{N} \delta\left(\left|z_{1}\right|^{2} \ldots\left|z_{l}\right|^{2}-\operatorname{det}\left[\mathbf{1}-\hat{T} \sum_{j=1}^{l} q_{j}\right]\right) .
\end{aligned}
$$

Finally, we introduce $\hat{Q}_{l}=\sum_{i=1}^{l} q_{i}$ and observe that Equ. (833) aquires the form

$$
\begin{gathered}
\mathcal{P}(\{z\}) \propto|\Delta(\{z\})|^{2} \int\left(\prod_{l=1}^{N-1} d \hat{Q}_{l}\right) \prod_{l=1}^{N} \delta\left(\left|z_{1}\right|^{2} \ldots\left|z_{l}\right|^{2}-\operatorname{det}\left(\mathbf{1}-\hat{T} \hat{Q}_{l}\right)\right) \\
\times \prod_{i=1}^{N} \int d^{2} \mathbf{a} \delta\left(\hat{Q}_{i}-\hat{Q}_{i-1}-\mathbf{a} \otimes \mathbf{a}^{\dagger}\right),
\end{gathered}
$$

where the matrices $\hat{Q}_{l}$ are considered to be unconstrained $N \times N$ Hermitian. We also used the orthonormality condition $\hat{Q}_{N}=\mathbf{1}$ as well as the convention $\hat{Q}_{0}=\mathbf{0}$.

\subsection{Rank-1 deviations from unitarity}

The remaining integration over the vectors $\mathbf{a}_{\mathbf{i}}$ still requires a quite lengthy calculation. The general case will be considered in the next section. A particular case which can be solved relatively easy is the case of a rank-one deviation from Hermiticity: $M=1$, corresponding to a system with only one open channel. This example is quite instructive and the exposition below follows 62. Evaluating the integrals Eq. (84) quite straightforwardly we arrive at a very simple expression:

$$
\mathcal{P}(\{z\}) \propto T^{1-N}|\Delta(\{z\})|^{2} \delta\left(1-T-\left|z_{1}\right|^{2} \ldots\left|z_{N}\right|^{2}\right) .
$$

provided $0 \leq\left|z_{l}\right| \leq 1$ for all eigenvalues, and zero otherwise. Here $0<T<1$ is the only non-zero eigenvalue of the deviation matrix.

Eq. 855) can be used to extract all n-point correlation functions defined as in Eq. ( 111). To achieve this it is convenient to use the Mellin transform with respect to the variable $\zeta=1-T$ :

$$
\tilde{R}_{n}\left(s ;\{z\}_{n}\right)=\int_{0}^{\infty} d \zeta \zeta^{s-1}\left[(1-\zeta)^{N-1} R_{n}\left(\{z\}_{n}\right)\right] .
$$


It is easy to notice that such a transform brings $\mathcal{P}(\{z\})$ to the form suitable for exploitation of the orthogonal polynomial method [49. The corresponding polynomials $p_{k}(z)=z^{k} \sqrt{k+s}$ are orthonormal with respect to the weight $f(z)=$ $|z|^{2(s-1)} / \pi$ inside the unit circle $|z| \leq 1$. Following the standard route we find:

$$
\tilde{R}_{n}\left(s ;\{z\}_{n}\right) \propto \frac{\left.\operatorname{det}\left[K\left(z_{i}, z_{j}^{*}\right)\right]\right|_{(i, j)=1, \ldots, n}}{s(s+1) \ldots(s+N-1)}
$$

where the kernel is

$$
\begin{aligned}
K\left(z_{1}, z_{2}^{*}\right) & =\left(f\left(z_{1}\right) f\left(z_{2}\right)\right)^{1 / 2} \sum_{k=0}^{N-1} p_{k}\left(z_{1}\right) p_{k}\left(z_{2}^{*}\right) \\
& =\left.\frac{|x|^{s-1}}{\pi}\left(s \phi(x)+x \frac{d}{d x} \phi(x)\right)\right|_{x=z_{1} z_{2}^{*}}
\end{aligned}
$$

and $\phi(x)=\left(x^{N}-1\right) /(x-1)$. Thus, the expression Eq.(87) can be rewritten as:

$$
\begin{aligned}
\tilde{R}_{n}\left(s ;\{z\}_{n}\right) & \propto \frac{\prod_{l=1}^{n}\left|z_{l}\right|^{2(s-1)}}{s(s+1) \ldots(s+N-1)} \sum_{l=0}^{n} s^{l} q_{l}\left(\{z\}_{n}\right) ; \\
q_{0}\left(\{z\}_{n}\right) & =\left.\operatorname{det}\left[\left.x \frac{d}{d x} \phi(x)\right|_{x=\left(z_{i} z_{j}^{*}\right)}\right]\right|_{i, j=1, \ldots, n} \\
& \ldots \\
q_{n}\left(\{z\}_{n}\right) & =\left.\operatorname{det}\left[\left.\phi(x)\right|_{x=\left(z_{i} z_{j}^{*}\right)}\right]\right|_{i, j=1, \ldots, n}
\end{aligned}
$$

and can easily be Mellin-inverted yielding finally the original correlation functions in the following form:

$\left.\left.R_{n}\left(\{z\}_{n}\right)\right|_{\left|z_{n}\right| \leq 1} \propto T^{1-N} \theta(T-1+a) \sum_{l=0}^{n} q_{l}\left(\{z\}_{n}\right)\left(\frac{d}{d a} a\right)^{l}\left[\frac{1}{a}\left(1-\frac{1-T}{a}\right)\right]^{N-1}\right|_{a=\prod_{i=1}^{n}\left|z_{i}\right|^{2}}$

where $\theta(x)=1$ for $x \geq 0$ and zero otherwise. This equation is exact for arbitrary $N$.

Let us now investigate the limit $N \gg n \geq l$ and use, to the leading order:

$$
\left(\frac{d}{d a} a\right)^{l}\left[\frac{1}{a}\left(1-\frac{\xi}{a}\right)\right]^{N-1} \approx\left(\frac{N \xi}{a-\xi}\right)^{l} \frac{1}{a}\left(1-\frac{\xi}{a}\right)^{N-1} \quad, \quad \xi=1-T .
$$

This allows one to rewrite the correlation function as a determinant:

$$
\begin{aligned}
R_{n}\left(\{z\}_{n}\right) & \propto T^{1-N} \frac{1}{a}\left(1-\frac{1-T}{a}\right)^{N-1} \theta(T-1+a) \\
& \times\left.\operatorname{det}_{i, j=1, \ldots, n}\left(\frac{N(1-T)}{a-1+T} \phi(x)+x \frac{d}{d x} \phi(x)\right)\right|_{x=z_{i} z_{j}^{*}} .
\end{aligned}
$$

Further simplifications occur after taking into account that the eigenvalues $z_{i}$ are expected to concentrate typically at distances of order of $1 / N$ from the unit circle. Introduce new variables $y_{i}, \theta_{i}$ according to $z_{i}=\left(1-y_{i} / N\right) e^{i \theta_{i}}$ and consider $y_{i}$ to be of the order of unity when $N \rightarrow \infty$. As to the phases $\theta_{i}$ their typical separation scales as: $\theta_{i}-\theta_{j}=O(1 / N)$. Now it is straightforward to perform the limit $N \rightarrow \infty$ explicitly and bring Eq. (90) to the final form:

$R_{n}\left(\{z\}_{n}\right) \propto e^{-g \sum_{i=1}^{n} y_{i}} \operatorname{det}\left[\int_{-1}^{1} d \lambda(\lambda+g) e^{-\frac{i}{2} \lambda\left(N\left(\theta_{i}-\theta_{j}\right)-i\left(y_{i}+y_{j}\right)\right)}\right]_{i, j=1, \ldots, n}$ 
with $g=2 / T-1 .^{+}$

The expression above coincides in every detail with that for rank-one deviations from Hermiticity, see Eqs.4243) provided one fixes before the rescaled coupling constant $g$ and then remembers that the mean linear density of phases $\theta_{i}$ along the unit circle is $\nu=1 /(2 \pi)$. The non-unitary matrices provide therefore an alternative way to address analytically "local-in-spectrum" universal features arising in the theory of chaotic scattering. Sometimes to deal with subunitary matrices is technically more advantageous than with their non-Hermitian counterparts. As an illustration of this statement we use subunitary matrices for extracting the distribution $f(\Gamma)$ of the width $\Gamma=2 \operatorname{Im} z$ of the most narrow resonance among $n=W / \Delta>>1$ falling in a narrow window $[E-W / 2, E+W / 2]$ in the vicinity of a given point $E$ in the spectrum. The latter quantity is of great interest in the theory of random lasing [54] since it is related to fluctuations of the lasing threshold. The functional form of the distribution was found in those papers by employing plausible qualitative arguments of virtual statistical independence of the widths of the neighbouring resonances. For the simplest single-channel case we are able to show analytically that [57]

$$
f(\Gamma)=\frac{\pi g n}{\Delta} e^{-\pi g n \Gamma / \Delta}
$$

The general case can be treated very similar. The distribution Eq.(91) is exactly that suggested in [54, but with renormalized effective coupling $g=\frac{1}{2 \pi \nu}\left(\gamma+\gamma^{-1}\right)$ replacing the combination $\gamma / 2 \pi \nu$. We see that the two expressions coincide only within the weak coupling limit $\gamma \rightarrow 0$, whereas the difference amounts to the factor 2 in the exponent for the case of perfect coupling $\gamma=1$. Let us briefly comment on the way of deriving the distribution Eq.(91). Instead of extracting such a quantity from the joint probability density Eq. (31) we find it technically easier to consider its counterpart Eq. (85) and interprete the parameter $N$ in Eq. (85) as the number $n$ of resonances in the window. We know that in the limit $n>>1$ the eigenvalues $z_{k}=r_{k} e^{\theta_{k}}$ are situated in a narrow vicinity of the unit circle and their statistics is indistinguishable from that of the complex eigenenergies $\mathcal{E}$, when the latter are considered locally, i.e. on the distances comparable with the mean spacing $\Delta$. In particular, the distances $1-r_{k}$ from the unit circle should be interpreted as the widths of the resonances. To calculate the distribution Eq. (91) we first notice that the form of Eq.(85) allows one to integrate out the phases $\theta_{i}$ by noticing that:

$$
\int_{0}^{2 \pi} \frac{d \theta_{1}}{2 \pi} \ldots \int_{0}^{2 \pi} \frac{d \theta_{n}}{2 \pi} \prod_{k<j}^{1 \ldots n}\left|r_{k} e^{i \theta_{k}}-r_{j} e^{i \theta_{j}}\right|^{2}=\operatorname{Per}\left(r_{1}^{2}, \ldots, r_{n}^{2}\right)
$$

where we denoted $\operatorname{Per}\left(x_{1}, \ldots, x_{n}\right)=\sum_{\{\alpha\}} x_{1}^{\alpha_{1}} \ldots x_{n}^{\alpha_{n}}$, and the summation goes over all possible permutations $\{\alpha\}=\left(\alpha_{1}, \ldots, \alpha_{n}\right)$ of the set $1, \ldots, n$ (in fact here we deal with an object known as "permanent", hence the notation). In this way we come to the joint probability density of the radial coordinates only. Such a density written in terms of the variables $R_{i}=r_{i}^{2}$ has the following form

$$
\mathcal{P}_{T}\left(R_{1}, \ldots, R_{n}\right) \propto T^{1-N} \operatorname{Per}\left(R_{1}, \ldots, R_{n}\right) \delta\left(1-T-R_{1} \ldots R_{n}\right) .
$$

Identifying resonance widths with the distances $1-r_{i}$, the probability distribution of the resonance $r_{1}$ closest to the unit circle: $r_{1}>r_{2}, r_{3}, \ldots, r_{n}$ is naturally described in

+ The coupling constants $g_{a}$ in this section are rescaled and correspond to $g_{a} / \pi \nu(X)$ of subsection 2.2.There is no difference at $X=0$. The coupling constants are always $\geq 1$. 
terms of the function:

$$
p_{T}\left(R_{1}\right)=\int_{0}^{R_{1}} d R_{2} \ldots \int_{0}^{R_{1}} d R_{n} \mathcal{P}_{T}\left(R_{1}, \ldots, R_{n}\right) .
$$

Again it is convenient to multiply this expression with $T^{N-1}$ and to perform the Mellin transform with respect to $1-T$. Then the integration over $R$-variables is simple to perform and after inverting the Mellin transform one finds:

$$
p_{T}\left(R_{1}\right)=\theta\left(R_{1}^{n}-1+T\right) \frac{1}{T^{n-1}} \frac{d}{d R_{1}}\left[R_{1}^{n / 2}\left(1-\frac{1-T}{R_{1}^{n}}\right)\right]^{n-1}
$$

The distribution Eq. (91) follows from Eq.(95) after rescaling $R_{1}=1-2 y / n, y=\pi \Gamma / \Delta$, performing the limiting procedure $n \rightarrow \infty$ and remembering $\Delta=2 \pi / n$.

\subsection{Rank $M>1$ deviations from unitarity}

Coming back to the general case we find it convenient to change: $\hat{T}^{1 / 2} \hat{Q}_{l} \hat{T}^{1 / 2} \rightarrow \hat{Q}_{l}$

and separate integration over eigenvalues and eigenvectors of matrices $\hat{Q}_{l}$. The latter can be performed in a recursive way $l \rightarrow l+1$, with the multiple use of the Itzykson-Zuber-Harish-Chandra Eq.(28) formula. After quite an elaborate manipulation, demonstrated in detail in appendix A2 one finally arrives at the following representation:

$$
\begin{array}{r}
\mathcal{P}(\{z\}) \propto \frac{\operatorname{det}^{M-N}(\hat{T})}{\operatorname{det}(\mathbf{1}-\hat{T}) \prod_{c_{1}<c_{2}}\left(T_{c_{1}}-T_{c_{2}}\right)} \prod_{c_{1}<c_{2}}\left(\frac{\partial}{\partial \tau_{c_{1}}}-\frac{\partial}{\partial \tau_{c_{2}}}\right) \\
\times \int d \hat{\lambda} e^{-i \operatorname{Tr} \hat{\tau} \hat{\lambda}}|\Delta(\{z\})|^{2} \prod_{k=1}^{N} f\left(\ln \left|z_{k}\right|^{2}, \hat{\lambda}\right),
\end{array}
$$

where we defined the diagonal matrices of size $M$ as: $\hat{\tau}=\operatorname{diag}\left(\tau_{1}, \ldots, \tau_{M}\right), \hat{\lambda}=$ $\operatorname{diag}\left(\lambda_{1}, \ldots, \lambda_{M}\right)$ and used the notations: $\tau_{c}=\ln \left(1-T_{c}\right)$ and

$$
f(a, \hat{\lambda})=i^{M-1} \sum_{q=1}^{M} \frac{e^{i \lambda_{q} a}}{\prod_{s(\neq q)}\left(\lambda_{q}-\lambda_{s}\right)} .
$$

The distribution Eq. (96) is written for $\left|z_{k}\right|^{2} \leq 1$ for any $k=1, \ldots, N$ and vanishes otherwise. It shows great similarity to the pair of equations (2530) describing eigenvalues of matrices deviating from Hermiticity. The remarkable feature of the distribution Eq. (96) is that it allows for calculation of all $n$-point correlation functions for arbitary $N, n, M$ with help of the method of orthogonal polynomials. Again, the particular case $M=1[\underline{62}$ is quite instructive and can be recommended to follow first for understanding of the general formulae outlined below.

To this end, we write

$$
|\Delta(\{z\})|^{2} \prod_{k=1}^{N} f\left(\ln \left|z_{k}\right|^{2}, \hat{\lambda}\right)=\prod_{k=1}^{N} N_{k}(\hat{\lambda}) \operatorname{det}\left[\sum_{n=1}^{N} \frac{\left(z_{i} z_{j}^{*}\right)^{n-1}}{N_{n}(\hat{\lambda})} f\left(\ln \left|z_{j}\right|^{2}, \hat{\lambda}\right)\right]_{i, j=1, \ldots N}
$$

where the constants $N_{n}(\hat{\lambda})$ are provided by the orthonormality condition:

$$
\int_{|z|^{2} \leq 1} d^{2} z z^{m-1}\left(z^{*}\right)^{n-1} f\left(\ln |z|^{2}, \hat{\lambda}\right)=\delta_{m, n} N_{n}(\hat{\lambda})
$$

which yields after a simple calculation $N_{n}(\hat{\lambda})=\pi \prod_{c=1}^{M} \frac{1}{\left(n+i \lambda_{c}\right)}$. 
Now, by applying the standard machinery of orthogonal polynomials 49] one can find the correlation function:

$$
R_{n}\left(z_{1}, \ldots, z_{n}\right)=\frac{N !}{(N-n) !} \int d^{2} z_{n+1} \ldots d^{2} z_{N} \mathcal{P}(\{z\})
$$

as given by:

$$
R_{n}\left(z_{1}, \ldots, z_{n}\right) \propto \hat{\mathcal{D}} \int d \hat{\lambda} e^{-i \operatorname{Tr} \hat{\tau} \hat{\lambda}} \prod_{k=1}^{N} N_{k}(\hat{\lambda}) \operatorname{det}\left[K\left(z_{i}, z_{j}^{*} ; \hat{\lambda}\right)\right]_{(i, j)=1, \ldots, n}
$$

where the kernel $K$ is defined as:

$$
K\left(z_{1}, z_{2}^{*} ; \hat{\lambda}\right)=\frac{1}{\pi} \sum_{n=1}^{N} \operatorname{det}(i \hat{\lambda}+n \mathbf{1})\left(z_{1} z_{2}^{*}\right)^{n-1} f\left(\ln \left|z_{2}\right|^{2}, \hat{\lambda}\right)
$$

and the differential operator $\hat{\mathcal{D}}$ is just the expression in front of the $\lambda$ - integral in Eq.(96). It is worth noting that we used a version of the kernel which is not symmetric with respect to its arguments $z_{1}, z_{2}$. This choice is legitimate, though unconventional, and is dictated mainly by our wish to avoid square roots which may induce complications when working with complex-valued expressions.

In principle, all $\lambda$ - integrations in Eq.(101) can be performed explicitly and the resulting formulae provide the desired general solution of the problem. However, for arbitary $N, M, n$ the results obtained in that way are still quite cumbersome. Some examples are given in [63. In the theory of quantum chaotic scattering we, however, expect a kind of universality in the semiclassical limit. Translated to the random matrix language such a limit corresponds to $N \rightarrow \infty$ at fixed $n, M$. Still, extracting the asymptotic behaviour of the correlation function $R_{n}\left(z_{1}, \ldots, z_{n}\right)$ from Eq. (101) in such a limit is not a completely straightforward task. A useful trick is to notice that Eq. (101) can be rewritten as:

$$
\begin{aligned}
& R_{k}\left(z_{1}, \ldots, z_{n}\right) \propto \sum_{q_{1}=1, \ldots, q_{n}=1}^{M} F_{q_{1}, \ldots, q_{n}}\left(\left\{T_{c} ; z\right\}\right) \\
& F_{q_{1}, \ldots, q_{n}}\left(\left\{T_{c} ; z\right\}\right)=\mathcal{B}\left\{T_{c}\right\} \operatorname{det}\left[\sum_{k=1}^{N}\left(\frac{-\partial}{\partial \tau_{1}}+k\right) \ldots\left(\frac{-\partial}{\partial \tau_{M}}+k\right)\left(z_{i} z_{j}^{*}\right)^{k-1}\right]_{i, j=1, \ldots, n} \\
& \times \prod_{c_{1}<c_{2}}^{1 \ldots M}\left(\frac{\partial}{\partial \tau_{c_{1}}}-\frac{\partial}{\partial \tau_{c_{2}}}\right) \int \prod_{c=1}^{M}\left(d \lambda_{c} \frac{\exp -i \lambda_{c} \tau_{c}}{\prod_{l=1}^{N}\left(l+i \lambda_{c}\right)}\right) \frac{e^{-i \sum_{j=1}^{n} \lambda_{q_{j}} \ln \left|z_{j}\right|^{2}}}{\prod_{j=1}^{n} \prod_{s\left(\neq q_{j}\right)}^{1 \ldots M}\left(\lambda_{q_{j}}-\lambda_{s}\right)}
\end{aligned}
$$

where we used the notation:

$$
\mathcal{B}\left\{T_{c}\right\}=\frac{1}{\prod_{c_{1}<c_{2}}\left(T_{c_{1}}-T_{c_{2}}\right)} \prod_{c=1}^{M} \frac{T_{c}^{M-N}}{\left(1-T_{c}\right)} .
$$

Introducing now the auxiliary differential operator $\hat{\mathcal{D}}_{q_{1}, \ldots, q_{n}}=\prod_{j=1}^{n} \prod_{s\left(\neq q_{j}\right)}^{1 \ldots M}\left(\frac{\partial}{\partial \tau_{q_{j}}}-\frac{\partial}{\partial \tau_{s}}\right)$ and considering its action upon the ratio $F_{q_{1}, \ldots, q_{n}} / \mathcal{B}\left\{T_{c}\right\}$ one can satisfy oneself that in the limit $N \gg M, n$ the leading contribution to $F_{q_{1}, \ldots, q_{n}}$ is given by:

$$
F_{q_{1}, \ldots, q_{n}} \propto \prod_{c=1}^{M} \theta\left(1-\tilde{T}_{c}\right) \frac{\left(1-\tilde{T}_{c}\right)}{\left(1-T_{c}\right)}\left(\frac{\tilde{T}_{c}}{T_{c}}\right)^{N-M} \prod_{j=1}^{n} \prod_{s\left(\neq q_{j}\right)}^{1 \ldots M}\left(\frac{1}{T_{q_{j}}}-\frac{1}{T_{s}}\right)^{-1} \operatorname{det}\left[K\left(z_{i}, z_{j}^{*} ;\left\{\tilde{T}_{c}\right\}\right)\right]_{(i, j)=1, \ldots, n}
$$


where the kernel is given by

$$
\begin{aligned}
K\left(z_{i}, z_{j}^{*} ;\left\{\tilde{T}_{c}\right\}\right) & =\sum_{k=1}^{N} \prod_{c=1}^{M}\left[(N-M) \frac{1-\tilde{T}_{c}}{\tilde{T}_{c}}+k-1\right]\left(z_{i} z_{j}^{*}\right)^{k-1} \\
& =\left.\prod_{c=1}^{M}\left[(N-M) \frac{1-\tilde{T}_{c}}{\tilde{T}_{c}}+x \frac{d}{d x}\right] \frac{1-x^{N}}{1-x}\right|_{x=z_{i} z_{j}^{*}}
\end{aligned}
$$

and we used the notation: $\tilde{T}_{c}=1-\exp \left(\tau_{c}-\sum_{j=1}^{n} \delta_{q_{j}, c} \ln \left|z_{j}\right|^{2}\right)$.

Further simplifications occur after taking into account that the eigenvalues $z_{i}$ are, in fact, concentrated typically at distances of order of $1 / N$ from the unit circle. Then it is natural to introduce new variables $y_{i}, \phi_{i}$ according to $z_{i}=\left(1-y_{i} / N\right) e^{i \theta_{i}}$ and consider $y_{i}$ to be of the order of unity when $N \rightarrow \infty$. First of all, one immediately finds that:

$$
\lim _{N \rightarrow \infty} \prod_{c=1}^{M}\left(\frac{\tilde{T}_{c}}{T_{c}}\right)^{N-M}=\exp \left(-2 \sum_{j=1}^{n} y_{j} \frac{1-T_{q_{j}}}{T_{q_{j}}}\right)
$$

As to the phases $\theta_{i}$, we expect their typical separation scaling as: $\theta_{i}-\theta_{j}=O(1 / N)$. Now it is straightforward to perform explicitly the limit $N \rightarrow \infty$ in Eq. (106). Combining all factors together, one brings the correlation function Eq. (103) to the final form coinciding in every detail with that obtained for random GUE matrices deformed by a finite rank anti-Hermitian perturbation, see Eqs. (4243), with $g_{c}=2 / T_{c}-1$. One should only remember that the mean density of phases $\theta_{i}$ along the unit circle is $\nu=1 /(2 \pi)$ and take this factor into account replacing the (semicircular) density factor $\nu(x)$ whenever necessary after fixing the rescaled coupling constants $g_{a}$. This completes the proof of equivalence, in the large $\mathrm{N}$ limit, of spectral properties for finite-rank deviations from Hermiticity and from unitarity.

We have seen already that the correlation function of moments of characteristic polynomials played a very important role in the theory of non-Hermitian matrices. It is natural to consider similar objects for general subunitary matrices $\hat{A}$ as well. So far only the simplest objects of this kind were calculated [81:

$$
I\left(z_{1}, z_{2}\right)=\left\langle\left[\operatorname{det}\left(z_{1} \mathbf{1}-\hat{A}\right) \operatorname{det}\left(z_{2}^{*} \mathbf{1}-\hat{A}^{\dagger}\right)\right]^{n}\right\rangle_{A}
$$

where the angular brackets stand for the averaging over the probability density in Eq.(17). We present the final result, which has the form of a Hankel determinant, in terms of eigenvalues $G_{i}=1-T_{i}$ of $\hat{G}$ :

$$
\begin{aligned}
& I\left(z_{1}, z_{2}\right)=\prod_{j=0}^{n-1} \frac{(N+n+j) !}{j !(j+1) !(N+j) !} \operatorname{det}\left[f_{i+j}\right]_{i, j=0, \ldots, n-1} \\
& f_{i+j}=\sum_{k=0}^{N}\left(z_{1} z_{2}^{*}\right)^{N-k} \frac{(N+i+j-k) !(2 n-2+k-i-j) !}{(N+2 n-1) !} \sum_{1 \leq i_{1}<i_{2}<\ldots<i_{k} \leq N} G_{i_{1}} G_{i_{2}} \ldots G_{i_{k}} .
\end{aligned}
$$

The particular case $n=1$ of the above formula was presented in 62. In fact, the expression Eq. (109) is valid for any $G_{i}>0$, being not at all restricted to the subunitary case $0<G_{i}<1$. For random unitary matrices all $G_{i}=1$ and one can show that the expression above are indeed in agreement with the known result for the moments of characteristic polynomilas of unitary matrices [82, 83]. 


\section{Statistics of Non-Orthogonal Eigenvectors}

Let us denote $\left|R_{k}\right\rangle$ and $\left\langle L_{k}\right|$ to be the right and the left eigenvectors of the matrix $\mathcal{H}$ corresponding to the eigenvalue $z_{k}$. This means

$$
\begin{array}{ll}
\mathcal{H}\left|R_{k}\right\rangle=z_{k}\left|R_{k}\right\rangle \quad & , \quad\left\langle L_{k}\right| \mathcal{H}=\left\langle L_{k}\right| z_{k} \\
\mathcal{H}^{\dagger}\left|L_{k}\right\rangle=z_{k}^{*}\left|L_{k}\right\rangle & , \quad\left\langle R_{k}\right| \mathcal{H}^{\dagger}=\left\langle R_{k}\right| z_{k}^{*} .
\end{array}
$$

These eigenvectors form a complete, bi-orthogonal set and can be normalized to satisfy

$$
\left\langle L_{m} \mid R_{n}\right\rangle=\delta_{m n}
$$

The most natural way to characterize the non-orthogonality of the eigenvectors is to consider the statistics of the overlap matrix $\mathcal{O}_{m n}=\left\langle L_{m} \mid L_{n}\right\rangle\left\langle R_{m} \mid R_{n}\right\rangle$. This matrix naturally enters many calculations that operate with non-Hermitian Hamiltonians, e.g. in the description of the particle escape from the scattering region ("norm leakage" 84]). Moreover, the entries $O_{n m}$ have a direct physical meaning for lasing media: diagonal ones are the so-called Petermann (or "excess noise") factors accessible experimentally, whereas the off-diagonal entries represent cross-correlations between the thermal or quantum noise emitted into different eigenmodes 60.

Thus, two correlation functions [53]: the diagonal one

$$
O(z)=\left\langle\frac{1}{N} \sum_{n} \mathcal{O}_{n n} \delta\left(z-z_{k}\right)\right\rangle_{\mathcal{H}_{N}}
$$

and the off-diagonal one

$$
O\left(z, z^{\prime}\right)=\left\langle\frac{1}{N} \sum_{n \neq m} \mathcal{O}_{n m} \delta\left(z-z_{n}\right) \delta\left(z^{\prime}-z_{m}\right)\right\rangle_{\mathcal{H}_{N}}
$$

are natural characteristics of average non-orthogonality of eigenvectors corresponding to the resonances whose positions in the complex plane are close to the complex energy $z$. Here $\delta(z)$ stands for the two-dimensional $\delta$-functions of the complex variable $z$. Let us note that for any ensemble with orthogonal eigenvectors and complex eigenvalues $z$ (e.g. for the so-called normal matrices) one obviously has $O(z)$ equal to the mean density of complex eigenvalues. For the same situation the off-diagonal correlator vanishes: $O\left(z_{1}, z_{2}\right) \equiv 0$.

Both diagonal and off-diagonal eigenvector correlators were introduced and explicitly calculated for the strongly non-Hermitian (Ginibre) ensemble of nonHermitian matrices by Chalker and Mehlig [53]. For the ensemble $\mathcal{J}_{N}=H-i \hat{\Gamma}$ pertinent to chaotic scattering both types of eigenvector correlators were found for the regime of very strongly overlapping resonances when widths typically much exceed the mean separation [56. We already discussed that such a regime corresponds to a large number $M \gg 1$ of open channels, and a kind of self-consistent Born approximation (or equivalent approximation schemes, see [55]) is justified in this case. A general non-perturbative expression for the diagonal correlator $O(z)$ can be extracted from the following relation [55]:

$$
O(z)=\frac{1}{\pi} \lim _{\epsilon \rightarrow 0}\left\langle\left(\operatorname{Tr} \frac{\epsilon}{\left(z-\mathcal{J}_{N}\right)\left(z^{*}-\mathcal{J}_{N}^{\dagger}\right)+\epsilon^{2} \mathbf{1}}\right)^{2}\right\rangle .
$$

Although a direct calculation of the right-hand side for weakly non-Hermitian matrices is difficult, Frahm et al. [54 managed to get to the result valid for any number 
$M$ of open channels by employing a kind of heuristic analytic continuation scheme suggested in [1] and known to reproduce the exact expression for the resonance widths distribution, like that in Eq.(74). Therefore we have good reasons to expect the result for the eigenvector correlator found in 54

$$
\langle K\rangle=O(z) / \rho(z)=1+\frac{2 \mathcal{F}(y)}{\mathcal{F}_{1}(y) \mathcal{F}_{2}(y)} \quad, \quad \mathcal{F}(y)=-\int_{0}^{y} d x \mathcal{F}_{1}(x) \frac{d}{d x} \mathcal{F}_{2}(x)
$$

where $y=\frac{2 \pi Y}{\Delta}$ and

$\mathcal{F}_{1}(y)=\frac{y^{M-1}}{(M-1) !} e^{-g y} \quad, \quad \mathcal{F}_{2}(y)=(-1)^{M} e^{g y}\left(\frac{d}{d y}\right)^{M}\left(e^{-g y} \frac{\sinh y}{y}\right)$

should be valid as well, though it is still desirable to verify this formula independently. No non-perturbative results for the off-diagonal eigenvalue correlator $O\left(z_{1}, z_{2}\right)$ were reported so far, to the best of our knowledge.

In a recent paper [57] exact non-perturbative expressions for both diagonal and off-diagonal eigenvector correlators were provided for the case of a system with broken time-reversal invariance coupled to continuum via a single open channel. The expression for the diagonal correlator was found to agree with the formula Eq. (116) and was verified by comparing the analytical expression with the result of direct numerical diagonalization of the matrices $\mathcal{J}_{N}$. In fact, numerically it is easier to compute smoothed averages, such as the mean number of eigenvalues $\left\langle n\left(L_{x}, L_{y}\right)\right\rangle$ inside the rectangle $A=\left(-L_{x} / 2 \leq \operatorname{Re} z \leq L_{x} / 2 \quad, \quad 0 \leq \operatorname{Im} z \leq L_{y}\right)$. Such a quantity can be obtained from the mean density $\rho(z)$ by a simple integration over the rectangular domain $A$. Similarly one can define the function $O_{1}\left(L_{x}, L_{y}\right)$ as the integral of the diagonal correlator $O(z)$ over the same domain. Numerically the latter quantity should be compared with $\sum_{z_{k} \in A} \mathcal{O}_{k k}$.

The formula for the off-diagonal correlator $O\left(z_{1}, z_{2}\right)$ found in [57] is given by:

$$
\begin{gathered}
O\left(z_{1}, z_{2}\right)=N\left(\frac{\pi \nu}{\Delta}\right)^{2} e^{-g\left(y_{1}+y_{2}\right)} \int_{-1}^{1} d \lambda_{1} \int_{-1}^{1} d \lambda_{2}\left(g+\lambda_{1}\right)\left(g+\lambda_{2}\right) e^{i \omega\left(\lambda_{1}+\lambda_{2}\right)} \\
\times e^{-y_{2}\left(\lambda_{1}-\lambda_{2}\right) / 2}\left[e^{y_{1}\left(\lambda_{1}-\lambda_{2}\right) / 2}-e^{-y_{1}\left(\lambda_{1}-\lambda_{2}\right) / 2}\right]
\end{gathered}
$$

where we introduced $\operatorname{Im} z_{1,2}=X_{1,2}=X \mp \Omega$, assumed that $\Omega \sim \Delta$ and denoted $\omega=\pi \Omega / \Delta$. Again, for numerical reasons it is easier to calculate the smoothed average $\sum_{z_{m}, z_{n}, \in A} \mathcal{O}_{m n}$ which should be compared with the corresponding integral of the function $O\left(z_{1}, z_{2}\right)$.

Let us now outline the derivation of the formulae Eqs. (116 118). The main idea is to use the fact that the complex eigenvalues $z_{k}$ of $\mathcal{H}_{\text {eff }}=\mathcal{J}_{N}$ are resonances, i.e. the poles of the $M \times M$ scattering matrix $\hat{S}(\mathcal{E})$ in the complex energy plane $\mathcal{E}$. Denoting by $V$ a (nonunitary!) matrix of (right) eigenvectors of $\mathcal{H}_{e f f}=V \hat{Z} V^{-1}$ we have, in particular, the identities:

$$
\begin{aligned}
& \left\{V^{\dagger}\left(\mathcal{H}_{e f f}-\mathcal{H}_{e f f}^{\dagger}\right) V\right\}_{m n}=\left(z_{n}-z_{m}^{*}\right)\left\{V^{\dagger} V\right\}_{m n} \\
& \left\{V^{-1}\left(\mathcal{H}_{\text {eff }}^{\dagger}-\mathcal{H}_{e f f}\right)\left[V^{-1}\right]^{\dagger}\right\}_{n m}=\left(z_{m}^{*}-z_{n}\right)\left\{V^{-1}\left[V^{-1}\right]^{\dagger}\right\}_{n m}
\end{aligned}
$$

Let us also note that according to the definition the entries of the overlap matrix $\mathcal{O}_{m n}$ are given in terms of $V$ as $\mathcal{O}_{m n}=\left\{V^{\dagger} V\right\}_{m n}\left\{V^{-1}\left[V^{-1}\right]^{\dagger}\right\}_{n m}$. 
Substituting the relation $E-\mathcal{H}_{\text {eff }}=V(E-\hat{Z}) V^{-1}$ into the formula for the scattering matrix Eq.(3) we can extract the behaviour of the scattering matrix elements when the (complex) energy parameter $\mathcal{E}$ approaches an individual pole:

$S_{a b}\left(\mathcal{E} \rightarrow z_{n}\right) \approx-2 \pi i\left(W^{\dagger} V\right)_{a n}\left(V^{-1} W\right)_{n b} \frac{1}{\mathcal{E}-z_{n}}$

A similar formula holds for the Hermitian conjugate of the scattering matrix:

$$
\left[S^{\dagger}\right]_{b a}\left(\mathcal{E} \rightarrow z_{m}\right) \approx 2 \pi i\left(W^{\dagger}\left[V^{-1}\right]^{\dagger}\right)_{b m}\left(V^{\dagger} W\right)_{m a} \frac{1}{\mathcal{E}^{*}-z_{n}^{*}}
$$

These two equations immediately yield:

$\operatorname{Tr}\left\{S\left(\mathcal{E}_{1} \rightarrow z_{n}\right)\left[S^{\dagger}\right]\left(\mathcal{E}_{2} \rightarrow z_{m}\right)\right\}=$

$\frac{4 \pi^{2}}{\left(\mathcal{E}_{1}-z_{n}\right)\left(\mathcal{E}_{2}^{*}-z_{n}^{*}\right)}\left(V^{-1} W W^{\dagger}\left[V^{-1}\right]^{\dagger}\right)_{n m}\left(V^{\dagger} W W^{\dagger} V\right)_{n m}$

Now we notice that $2 \pi i W W^{\dagger}=\mathcal{H}_{\text {eff }}-\mathcal{H}_{\text {eff }}^{\dagger}$ and further exploit the relations Eq. (119). This immediately yields the following relation between the trace of the S-matrix residues and the overlap matrices of the left and right eigenvectors:

$\operatorname{Tr}\left[\operatorname{Res} \hat{S}(\mathcal{E}) \operatorname{Res} \hat{S}^{\dagger}\left(\tilde{\mathcal{E}}^{*}\right)\right]_{\mathcal{E} \rightarrow z_{n}, \tilde{\mathcal{E}}^{*} \rightarrow z_{m}^{*}}=\left(z_{m}^{*}-z_{n}\right)\left(z_{n}-z_{m}^{*}\right) \mathcal{O}_{m n}$

Such a relation is of general validity, but for an arbitary number of open channels $M$ it seems to be of no obvious utility, due to difficulties in evaluating the ensemble average of the trace of the residues on the left-hand side. However for the particular case of a single open channel $M=1$ the scattering matrix coincides with its determinant and therefore has a different representation as (see Eq.(44)

$$
S(\mathcal{E})=\prod_{k=1}^{N} \frac{\mathcal{E}-z_{k}^{*}}{\mathcal{E}-z_{k}}, \quad \text { hence } \quad S^{\dagger}\left(\mathcal{E}^{*}\right)=\prod_{k=1}^{N} \frac{\mathcal{E}^{*}-z_{k}}{\mathcal{E}^{*}-z_{k}^{*}}
$$

In fact, this formula is model-independent and follows, up to an irrelevant "nonresonant" phase factor, from the requirement of $S$-matrix analyticity in the upper half-plane and unitarity for real energies. This expression substituted in Eq. (123) yields immediately the relation:

$$
\mathcal{O}_{m n}=\frac{\left(z_{n}-z_{n}^{*}\right)\left(z_{m}-z_{m}^{*}\right)}{\left(z_{n}-z_{m}^{*}\right)^{2}} \prod_{k(\neq n)}^{N} \frac{z_{n}-z_{k}^{*}}{z_{n}-z_{k}} \prod_{k(\neq m)}^{N} \frac{z_{m}^{*}-z_{k}}{z_{m}^{*}-z_{k}^{*}}
$$

giving the eigenvector non-orthogonality overlap matrix in terms of the complex eigenvalues $z_{k}$. This provides us with a possibility to find the diagonal and off-diagonal correlators, Eqs. (113114), by averaging $\mathcal{O}_{m n}$ over the known joint probability density of complex eigenvalues for the single-channel scattering system Eq. (31). Indeed, one may notice that

$O(z)=\frac{\tilde{\gamma}_{1}^{N-2}}{\gamma^{N-1}} e^{-\frac{1}{2}\left[N \gamma^{2}-(N-1) \tilde{\gamma_{1}}\right]} e^{-\frac{N}{4}\left(z^{2}+z^{* 2}\right)}\left\langle\operatorname{det}\left[\left(z-\mathcal{J}^{\dagger}\right)\left(z^{*}-\mathcal{J}\right)\right]\right\rangle_{\tilde{\mathcal{J}}_{N-1}}$

where $\tilde{\mathcal{J}}_{N-1}$ stands for the non-Hermitian matrix of the same type as $\mathcal{J}_{N}$ but of the lesser size $(N-1) \times(N-1)$, and with coupling $\gamma$ replaced by a modified coupling $\tilde{\gamma_{1}}=\gamma-\operatorname{Im} z$. Analogously

$$
\begin{gathered}
O\left(z_{1}, z_{2}\right)=\frac{{\tilde{\gamma_{2}}}^{N-3}}{\gamma^{N-1}} e^{-\frac{1}{2}\left[N \gamma^{2}-(N-2) \tilde{\gamma}_{2}\right]} e^{-\frac{N}{4} \sum_{n=1}^{2}\left(\mathcal{E}_{n}^{2}+z_{n}^{* 2}\right)}\left(z_{1}-z_{1}^{*}\right)\left(z_{2}-z_{2}^{*}\right) \\
\times\left\langle\operatorname{det}\left[\left(z_{1}-\mathcal{J}^{\dagger}\right)\left(z_{1}^{*}-\mathcal{J}^{\dagger}\right)\left(z_{2}-\mathcal{J}\right)\left(z_{2}^{*}-\mathcal{J}\right)\right]\right\rangle_{\tilde{\mathcal{J}}_{N-2}}
\end{gathered}
$$


where $\tilde{\mathcal{J}}_{N-2}$ is of the size $(N-2) \times(N-2)$, and with coupling $\gamma$ replaced by a modified coupling $\tilde{\gamma_{2}}=\gamma-\operatorname{Im} z_{1}-\operatorname{Im} z_{2}$.

In this way the problem is again reduced to calculating a correlation function of the characteristic polynomials of large non-Hermitian matrices as that in Eq. (333). The scaling limit $N \gg 1$ such that $\operatorname{Im} z_{1,2}=\Gamma_{1,2} \sim 2 \Omega=\operatorname{Re}\left(z_{1}-z_{2}\right) \sim \Delta \propto N^{-1}$ of the resulting expressions just yields the formulae Eqs.(116118) above.

\section{Conclusions and perspectives}

In the present paper we discussed properties of random matrix ensembles which can be naturally called non-Hermitian/non-unitary deformations of classical Hermitian/unitary ensembles.

Guided by the example of resonances in quantum chaotic scattering, we revealed the existence of a non-trivial regime of weak non-Hermiticity. The regime can be defined as that for which the imaginary part $\operatorname{Im} Z$ of a typical complex eigenvalue is of the same order as the mean eigenvalue separation $\Delta$ for the corresponding Hermitian counterpart $\hat{H}$. We systematically demonstrate that for such a regime the correlation functions of complex eigenvalues describe a non-trivial crossover from the Wigner-Dyson statistics of real eigenvalues typical for Hermitian/unitary random matrices to the Ginibre statistics in the complex plane typical for ensembles with strong non-Hermiticity. The latter is defined by the condition $<\operatorname{Tr} H^{2}>\propto<\operatorname{Tr} \Gamma^{2}>$ when $N \rightarrow \infty$. Another important new feature of the non-Hermitian matrices is the emerging non-orthogonality of eigenvectors, and we discussed a few results available in the literature.

Among challenging problems for future research we would like to mention extending our understanding to other symmetry classes of weakly non-Hermitian matrices, most importantly to complex symmetric and real asymmetric cases. In fact, the joint probability densities of complex eigenvalues for some particular cases are known, see e.g. 13, 79, but no progress in extracting any correlation function beyond the mean eigenvalue density was reported so far. For non-perturbative calculations (replica trick) of the mean eigenvalue density for various non-Hermitian ensembles see the recent paper 74. A consistent non-perturbative treatment of non-Hermitian matrices with "chiral" symmetry is still an open problem as well. Next to nothing is known about statistics of non-orthogonal eigenvectors for other classes of nonHermitian matrices, in particular, for the important case of the time-reversal invariant scattering.

Our considerations were mainly restricted to a Gaussian probability measure for the Hermitian counterparts of the considered matrices. An interesting open issue is the universality of the obtained results versus modification of the probability measures for Hermitian and anti-Hermitian parts. Relatively little is known in this direction and the available results are restricted by some specific types of the probability measure 44. Within the non-linear $\sigma$-model formalism one can demonstrate a kind of universality by non-rigorous, heuristic methods for matrices with independent entries. A challenging problem is to elevate our understanding of properties of almostHermitian random matrices to the level typical for their Hermitian counterparts.

Some interesting questions still remain to be answered even for non-Hermitian deformations of GUE matrices. To ensure their applications to the theory of chaotic scattering, we first have to improve our knowledge on eigenvector statistics. In 
particular, one needs to study the case of more than one channel and the problem of understanding fluctuations of the non-orthogonality overlap matrix $\mathcal{O}_{m n}$.

From all these points of view a most perspective direction seems to be the method of reducing various correlation functions of interest to products (and, in the general case, ratios!) of spectral determinants (characteristic polynomials). For Hermitian random matrices closely related objects were recently quite intensively treated by various analytical techniques [86] and the issue of universality proven to be amenable to a rigorous mathematical treatment 87 .

Recently, there was considerable interest in understanding interplay between the weak non-Hermiticity and Anderson localization [76] and in statistics of the resonances in systems with localized eigenfunctions 89. Clearly, this issue deserves further attention.

All questions about non-Hermitian matrices can equally be asked for matrices deviating from unitarity. Actually, some statistical properties of general subunitary matrices were under investigation recently as a model of scattering matrix for systems with absorption, see 88 . One may hope that this class of random matrices will find growing applications in the future.

\section{Acknowledgements}

The authors are much obliged to B.A.Khoruzhenko, B.Mehlig, M.Titov and K. Życzkowski for collaboration on different aspects of non-Hermitian random matrices discussed in the present paper. Communications and discussions with many colleagues over the years, especially with G.Akemann, Y.Alhassid, A.Garcia-Garcia, S.Grebenschikov, T.Guhr, I.Guarneri, F.Haake, G.Hackenbroich, T.Kottos, J.Keating, V.Mandelshtam, L.Pastur, D.Savin, H.Schomerus, T.Seligman, V.Sokolov, T.Wettig, H.-A.Weidenmueller, and J. Verbaarschot on various questions related to the subject are much appreciated.

This work was supported by SFB 237 "Unordnung und grosse Fluktuationen" and by EPSRC grant GR/13838/01 "Random matrices close to unitary or Hermitian" .

\section{Appendix A. Various integrals over the unitary group}

Appendix A.1. Itzykson-Zuber integral for the case of matrices having reduced rank

Denote $\mathbf{u}_{c}, c=1, \ldots, N$ columns of the unitary $N \times N$ matrix $\hat{U} \in U(N)$. They are orthonormal $N$-component complex vectors: $\mathbf{u}_{c_{1}}^{\dagger} \mathbf{u}_{c_{2}}=\delta_{c_{1} c_{2}}$. To evaluate the integral (28) for the case of a matrix $\Gamma$ of reduced rank $M<N$ (i.e. when only $M$ eigenvalues denoted by $\gamma_{1}, \ldots, \gamma_{M}$ are nonzero, the rest $N-M$ being zero) we notice that the combination $\operatorname{Tr} K U^{\dagger} \Gamma U=\sum_{c=1}^{M} \gamma_{c} \mathbf{u}_{c}^{\dagger} \hat{K} \mathbf{u}_{c}$ obviously depends only on the vectors $\mathbf{u}_{c}$ with $1 \leq c \leq M$. These column vectors form a $N \times M$ matrix which we denote $\hat{V}_{M}$, and the $\left(M-\right.$ component) rows of this matrix we denote as $\mathbf{v}_{j}^{\dagger}, j=1, \ldots, N$. Then $\operatorname{Tr} K U^{\dagger} \Gamma U=\sum_{j=1}^{N} k_{j}\left(\mathbf{v}_{j}^{\dagger} \hat{\gamma}_{M} \mathbf{v}_{j}\right)$ where we denoted $\hat{\gamma}=\operatorname{diag}\left(\gamma_{1}, \ldots, \gamma_{M}\right)$.

Consider the $M \times M$ matrix $\hat{I}_{M}(\mathbf{v})=\sum_{j=1}^{N} \mathbf{v}_{j} \otimes \mathbf{v}_{j}^{\dagger}$. It is easy to see that the conditions of mutual orthonormality of the $M$ vectors $\mathbf{u}_{c}, c=1, \ldots, M$ are equivalent to the condition of the matrix $\hat{I}_{M}(\mathbf{v})$ to be just the identity matrix: $\hat{I}_{M}(\mathbf{v})=\mathbf{1}_{M}$. 
Therefore the integration measure over the manifold of matrices $\hat{V}_{M}$ must be given by

$$
\begin{aligned}
d\left[\hat{V}_{M}\right] \propto \prod_{j=1}^{N} d^{2} \mathbf{v}_{j} \delta\left(\sum_{j=1}^{N} \mathbf{v}_{j} \otimes \mathbf{v}_{j}^{\dagger}-\mathbf{1}_{M}\right) \\
\propto \prod_{j=1}^{N} d^{2} \mathbf{v}_{j} \int\left[d \hat{F}_{M}\right] e^{i \operatorname{Tr} \hat{F}_{M}\left(\sum_{j=1}^{N} \mathbf{v}_{j} \otimes \mathbf{v}_{j}^{\dagger}-\mathbf{1}_{M}\right)}
\end{aligned}
$$

where we used the (matrix) Fourier-representation for the (matrix) $\delta$ - function, with $\hat{F}_{M}$ being the manifold of Hermitian $M \times M$ matrices.

These facts allow us to rewrite the IZHC integral for the present case as:

$$
\begin{aligned}
\int[d U]_{N} e^{-i \operatorname{Tr} K \hat{U}^{\dagger} \Gamma \hat{U}} \propto \int d \hat{F}_{M} e^{-i \operatorname{Tr} \hat{F}} \prod_{j=1}^{N} \int\left[d^{2} \mathbf{v}\right] e^{i \mathbf{v}^{\dagger}\left(\hat{F}_{M}-k_{j} \hat{\gamma}_{M}\right) \mathbf{v}} \\
\propto \int d \hat{F}_{M} e^{-i \operatorname{Tr} \hat{F}_{M}} \prod_{j=1}^{N} \operatorname{det}\left(\hat{F}_{M}-k_{j} \hat{\gamma}_{M}\right)^{-1}
\end{aligned}
$$

These integrals to be well-defined we assume a positive infinitesimal positive imaginary part added to $\hat{F}_{M}$ in the denominator. Now we introduce the Hermitian matrix $\hat{F}$ by $\hat{F}_{M}=\hat{\gamma}_{M}^{1 / 2} \hat{F} \hat{\gamma}_{M}^{1 / 2}$ which yields the Jacobian factor: $d \hat{F}_{M}=\left[\operatorname{det} \hat{\gamma}_{M}\right]^{M} d \hat{F}$. Diagonalizing $\hat{F}=U_{M}^{-1} \hat{\Lambda}_{M} U_{M}$ we use the matrix of eigenvalues $\hat{\Lambda}_{M}=\operatorname{diag}\left(\lambda_{1}, \ldots, \lambda_{M}\right)$ and $M \times M$ unitary matrices $U_{M}$ as new variables of integration, with the usual change in the measure as $d \hat{F}=\Delta^{2}\left(\hat{\Lambda}_{M}\right) d \hat{\Lambda}_{M}[d U]_{M}$. After these manipulations we arrive at the relation

$$
\begin{gathered}
\int[d U]_{N} e^{-i \operatorname{Tr} K U^{\dagger} \Gamma U} \propto\left[\operatorname{det} \hat{\gamma}_{M}\right]^{M-N} \int d \hat{\Lambda}_{M} \Delta^{2}\left(\hat{\Lambda}_{M}\right) \prod_{j=1}^{N} \prod_{c=1}^{M} \frac{1}{\lambda_{c}-k_{j}} \int[d U]_{M} e^{-i \operatorname{Tr} \hat{\gamma} U_{M}^{\dagger} \hat{\Lambda}_{M} U_{M}} \\
=\frac{\left[\operatorname{det} \hat{\gamma}_{M}\right]^{M-N}}{\Delta\left(\hat{\gamma}_{M}\right)} \int d \hat{\Lambda}_{M} \Delta\left(\hat{\Lambda}_{M}\right) \prod_{c=1}^{M} \prod_{j=1}^{N} \frac{1}{\lambda_{c}-k_{j}} \operatorname{det}\left[e^{-i \gamma_{c_{1}} \lambda_{c_{2}}}\right]_{c_{1}, c_{2}=1, \ldots, M}
\end{gathered}
$$

where we used the standard IZHC formula Eq.(28) for the integration over $U_{M}$. Finally, at the last step we use the symmetry of the integrand with respect to permutations of the arguments $\lambda_{1}, \ldots, \lambda_{M}$ and arrive at the required extension of the IZHC integral (29):

$$
\int[d U]_{N} e^{-i \operatorname{Tr} K U^{\dagger} \Gamma U} \propto M ! \frac{\operatorname{det} \gamma^{M-N}}{\Delta(\gamma)} \int d \hat{\Lambda}_{M} \Delta\left(\Lambda_{M}\right) \prod_{c=1}^{M} f_{c}\left(\lambda_{c}\right),
$$

where

$$
f_{c}\left(\lambda_{c}\right)=\frac{e^{-i \gamma_{c} \lambda_{c}}}{\prod_{j=1}^{N}\left(\lambda_{c}-k_{j}\right)}
$$

which is valid for the case when one of the matrices is of a reduced rank $M<N$. Let us finally satisfy oneself that when both matrices $K$ and $\Gamma$ are of the full rank: $M=N$ our expression Eq. A.4 reproduces exactly the familiar Itzykson-Zuber formula (28). To this end we use the Cauchy identity:

$$
\operatorname{det}\left(\frac{1}{\lambda_{c}-k_{j}}\right)_{c, j=1, N} \equiv \frac{\Delta(\Lambda) \Delta(K)}{\prod_{j=1}^{N} \prod_{c=1}^{N}\left(\lambda_{c}-k_{j}\right)}
$$


which allows us to write the write-hand side of (A.4 as

$$
\begin{aligned}
& \frac{i^{-N}}{\Delta(\Gamma) \Delta(K)} \int d \Lambda e^{-\sum_{i=1}^{N} \gamma_{i} \lambda_{i}} \operatorname{det}\left(\frac{1}{\lambda_{c}-k_{j}}\right)_{c, j=1, N} \\
& \equiv \frac{i^{-N}}{\Delta(\Gamma) \Delta(K)} \sum_{T}(-1)^{T} \prod_{c=1}^{N} \int d \lambda_{c} e^{-i \gamma_{c} \lambda_{c}} \frac{1}{\lambda_{c}-k_{j_{c}}}
\end{aligned}
$$

where the summation goes over all permutations $T=\left(k_{j_{1}}, \ldots, k_{j_{N}}\right)$ of the set $\left(k_{1}, \ldots, k_{N}\right)$, with the factors $(-1)^{T}$ standing for the signs of permutation. Each integration yields a factor: $i \exp \left(-i \gamma_{c} k_{j_{c}}\right)$. Taking into account the factors $(-1)^{T}$ allows one to present the result as a conventional Itzykson-Zuber determinant.

\section{Appendix A.2. Integration over unitary group for subunitary matrices}

We start from Eq. (83) and want to perform all integrations over matrices $\hat{Q}_{l}$ and complex vectors a. Rescaling all variables according to $\tilde{Q}_{i}=\sqrt{\hat{T}} \hat{Q}_{i} \sqrt{\hat{T}}$ with $\tilde{Q}_{0}=\mathbf{0}$ and $\tilde{Q}_{N}=\hat{T}$, and $\tilde{\mathbf{a}}=\sqrt{\hat{T}} \mathbf{a}$ and taking into account all Jacobians from the transformations we arrive at

$$
\begin{aligned}
\mathcal{P}(\{Z\}) & \propto|\Delta(\{Z\})|^{2} \int \prod_{i=1}^{N-1} d \tilde{Q}_{i} \prod_{i=1}^{N} \int d^{2} \tilde{\mathbf{a}} \delta\left(\tilde{Q}_{i}-\tilde{Q}_{i-1}-\tilde{\mathbf{a}} \otimes \tilde{\mathbf{a}}^{\dagger}\right) \\
& \times \prod_{l=1}^{N} \delta\left(\left|z_{1}\right|^{2} \ldots\left|z_{l}\right|^{2}-\operatorname{det}\left[\mathbf{1}-\tilde{Q}_{l}\right]\right) \operatorname{det} \hat{T}^{M-N}
\end{aligned}
$$

The following strategy is to diagonalize all matrices $\tilde{Q}_{i}$ as $\tilde{Q}_{i}=U_{i} \lambda_{i} U_{i}^{-1}$ with

$$
d \tilde{Q}_{i} \propto \prod_{j<k}\left(\lambda_{i}^{(j)}-\lambda_{i}^{(k)}\right)^{2} \prod_{l} d \lambda_{i}^{(l)} d \mu\left(U_{i}\right)
$$

and then perform the integrations over the eigenvectors $U_{i}$. We start with $\int d^{2} \mathbf{a} \delta\left(\tilde{Q}_{1}-\right.$ $\left.\mathbf{a} \otimes \mathbf{a}^{\dagger}\right)$. Since we integrate over all directions of the complex vector a the integral does not depend on $U_{1}$, i.e. the eigenvectors of $\tilde{Q}_{1}$ and is given by (cf. Eq. A.1.

$$
\begin{aligned}
\int d^{2} \mathbf{a} \delta\left(\tilde{Q}_{1}-\mathbf{a}\right. & \left.\otimes \mathbf{a}^{\dagger}\right) \propto \int d \Omega \exp \left(i \operatorname{Tr} \Omega \tilde{Q}_{1}\right) / \operatorname{det}(\epsilon+i \Omega) \\
& \propto \frac{1}{\prod_{j<k}\left(\lambda_{1}^{(j)}-\lambda_{1}^{(k)}\right)} \prod_{j<k}\left(-\frac{\partial}{\partial \lambda_{1}^{(j)}}+\frac{\partial}{\partial \lambda_{1}^{(k)}}\right) \prod_{l} \theta\left(\lambda_{1}^{(l)}\right) .
\end{aligned}
$$

Here we diagonalized the matrix $\Omega$ and integrated out the corresponding eigenvectors with the use the IZHC formula Eq.(28), with $\epsilon>0$ ( $\propto$ unity matrix) being an infinitesimal regularisation matrix. The result is a complicated expression in terms of $\delta$-functions and step functions. We now proceed to calculate the next factor in (A.6). Using again the IZHC integral two times we obtain

$$
\int d \mu\left(U_{1}\right) \int d^{2} \mathbf{a} \delta\left(\tilde{Q}_{2}-\tilde{Q}_{1}-\mathbf{a} \otimes \mathbf{a}^{\dagger}\right) \propto \frac{\operatorname{det}\left(\theta\left(\lambda_{2}^{(l)}-\lambda_{1}^{(m)}\right)\right)}{\prod_{j<k}\left(\lambda_{2}^{(j)}-\lambda_{2}^{(k)}\right)\left(\lambda_{1}^{(j)}-\lambda_{1}^{(k)}\right)}
$$

We used here that A.7 was independent of $U_{1}$ and we see that A.8 is independent of $U_{2}$. Using the latter fact on the next stage, we are able in this way to perform the integrations over all $\tilde{Q}_{i}$-diagonalizing matrices $U_{i}$. There remain the integrations over 
all the eigenvalues $\lambda_{i}^{(j)}$ of the matrices $\tilde{Q}_{i}$. The resulting expression for $\mathcal{P}(\{Z\})$ may now be written as

$$
\begin{gathered}
\mathcal{P}(\{Z\}) \propto \frac{\operatorname{det} \hat{T}^{M-N}}{\prod_{j<k}\left(T_{j}-T_{k}\right)}|\Delta(\{Z\})|^{2} \int \prod_{i=1}^{N-1} d Q_{i} \theta\left(\hat{T}-Q_{N-1}\right) \ldots \theta\left(Q_{2}-Q_{1}\right)\left[\Delta_{1} \theta\left(Q_{1}\right)\right] \\
\times \prod_{l=1}^{N} \delta\left(\left|z_{1}\right|^{2} \ldots\left|z_{l}\right|^{2}-\operatorname{det}\left[\mathbf{1}-Q_{l}\right]\right) .
\end{gathered}
$$

with diagonal $M \times M$ matrices $Q_{i}$, and the notations $d Q_{1}:=\prod_{i=1}^{M} d \lambda_{1}^{(i)}$

$$
\begin{aligned}
& \theta\left(Q_{2}-Q_{1}\right):= \prod_{i=1}^{M} \theta\left(\lambda_{2}^{(i)}-\lambda_{1}^{(i)}\right) \quad, \quad \operatorname{det}\left(\mathbf{1}-Q_{1}\right):=\prod_{i=1}^{M}\left(1-\lambda_{1}^{(i)}\right) \\
& \Delta_{1} \theta\left(Q_{1}\right):=\prod_{j<k}^{1 \ldots M}\left(-\frac{\partial}{\partial \lambda_{1}^{(j)}}+\frac{\partial}{\partial \lambda_{1}^{(k)}}\right) \prod_{l=1}^{M} \theta\left(\lambda_{1}^{(l)}\right) .
\end{aligned}
$$

An important simplification occurs if we go to logarithmic variables $\tau_{i}=\ln \left(\mathbf{1}-Q_{i}\right)$ (again these are diagonal $M \times M$ matrices with $\left.0 \geq \tau_{i} \geq \ln (\mathbf{1}-\hat{T})=\tau_{N}\right)$. We have

$$
\begin{aligned}
\delta\left(\left|z_{1}\right|^{2} \ldots\left|z_{l}\right|^{2}-\right. & \left.\operatorname{det}\left[\mathbf{1}-Q_{l}\right]\right) \theta\left(Q_{l}-Q_{l-1}\right)=\frac{1}{\operatorname{det}\left(\mathbf{1}-Q_{l}\right)} \delta\left(\sum_{j=1}^{l} \ln \left|z_{j}\right|^{2}-\sum_{k=1}^{M} \ln \left(1-\lambda_{l}^{(k)}\right)\right) \\
\times & \prod_{j=1}^{M} \theta\left(\ln \left(1-\lambda_{l-1}^{(j)}\right)-\ln \left(1-\lambda_{l}^{(j)}\right)\right)
\end{aligned}
$$

and there is the special relation

$$
\Delta_{1} \theta\left(Q_{1}\right)=\prod_{j<k}^{1 \ldots M}\left(\frac{\partial}{\partial \tau_{1}^{(j)}}-\frac{\partial}{\partial \tau_{1}^{(k)}}\right) \prod_{l=1}^{M} \theta\left(-\tau_{1}^{(l)}\right)=: \tilde{\Delta}_{1} \theta\left(-\tau_{1}\right) .
$$

Taking into account the Jacobian from the transformation our joint density takes the form

$$
\begin{gathered}
\mathcal{P}(\{Z\}) \propto \frac{\operatorname{det} \hat{T}^{M-N}|\Delta(\{Z\})|^{2}}{\operatorname{det}(\mathbf{1}-\hat{T}) \prod_{j<k}\left(T_{j}-T_{k}\right)} \int \prod_{i=1}^{N-1} d \tau_{i}\left[\tilde{\Delta}_{1} \theta\left(-\tau_{1}\right)\right] \theta\left(\tau_{1}-\tau_{2}\right) \ldots \theta\left(\tau_{N-1}-\tau_{N}\right) \\
\times \prod_{l=1}^{N} \delta\left(\ln \left|z_{1}\right|^{2}+\ldots+\ln \left|z_{l}\right|^{2}-\operatorname{Tr} \tau_{l}\right)
\end{gathered}
$$

Now we see that the operator $\tilde{\Delta}_{1}$ can be shifted by partial integration, it commutes with $\operatorname{Tr} \tau_{1}$ and then acts as $\tilde{\Delta}_{2}$ on $\theta\left(\tau_{1}-\tau_{2}\right)$. This argument can be repeated, such that finally the operator $\tilde{\Delta}_{N}$ appears in front of the remaining integral. Changing again the integration variables we obtain

$\mathcal{P}(\{Z\}) \propto \frac{\operatorname{det} \hat{T}^{M-N}|\Delta(\{Z\})|^{2}}{\operatorname{det}(\mathbf{1}-\hat{T}) \prod_{j<k}\left(T_{j}-T_{k}\right)} \tilde{\Delta} \int \prod_{l=1}^{N}\left[d \tau_{l} \theta\left(-\tau_{l}\right) \delta\left(\ln \left|z_{l}\right|^{2}-\operatorname{Tr} \tau_{l}\right)\right] \delta\left(\sum_{i=1}^{N} \tau_{i}-\tau\right)$

with $\tau:=\ln (\mathbf{1}-\hat{T})$ and $\tilde{\Delta}:=\prod_{j<k}^{1 \ldots M}\left(\frac{\partial}{\partial \tau^{(j)}}-\frac{\partial}{\partial \tau^{(k)}}\right)$. Writing down the Fourier representations of the $\delta$-functions in (A.13) the integrations over the diagonal $M \times M$ matrices $\tau_{i}$ can mainly be performed yielding

$$
\int_{\left\{\tau_{i}<0\right\}} \prod_{l=1}^{N}\left[d \tau_{l} \delta\left(\ln \left|z_{l}\right|^{2}-\operatorname{Tr} \tau_{l}\right)\right] \delta\left(\sum_{i=1}^{N} \tau_{i}-\tau\right)=\int\left[\frac{d \hat{\omega}}{2 \pi}\right] \exp (-i \operatorname{Tr} \hat{\omega} \tau) \prod_{l=1}^{N} f\left(\ln \left|z_{l}\right|^{2}, \hat{\omega}\right)(
$$


with

$f(a, \hat{\omega})=\int \frac{d \Omega}{2 \pi} \frac{\exp (i \Omega a)}{\prod_{k=1}^{M}\left(-i \Omega+i \omega_{k}+\epsilon\right)}=\sum_{k=1}^{M} \frac{\exp \left(i \omega_{k} a\right)}{\prod_{j(\neq k)}\left(-i \omega_{k}+i \omega_{j}\right)} \theta(-a)$

and with an infinitesimal $\epsilon>0$. The integration over $\Omega$ can be performed in the complex plane yielding the restriction $a<0$, i.e. in A.13 all $z_{i}$ lying inside the unit circle $\left(\ln \left|z_{i}\right|^{2}<0\right)$. Thus we have derived the full joint density of eigenvalues of subunitary matrices (96), which can be used to derive all correlation functions.

\section{Appendix B. Derivation of Eq.(32)}

Notice that in the limit $N \rightarrow \infty$ we can replace $N$ in the exponent in Eq. (24) by $N-n$. Then, similar to the Gaussian case, see Eq.(22), we first write:

$$
\begin{aligned}
& R_{n}(\{z\}) \propto \frac{e^{-\frac{N-n}{2}\left[\sum_{j=1}^{n} \operatorname{Re} z_{j}^{2}+\sum_{c=1}^{M} \gamma_{c}^{2}\right]}|\Delta(\{z\})|^{2}}{\Delta(\gamma) \operatorname{det} \gamma^{N-M}} \int d^{2}\{\xi\} e^{-\frac{N-n}{2} \sum_{l=1}^{N-n} \operatorname{Re} \xi_{l}^{2}}|\Delta(\{\xi\})|^{2} \\
& \times \prod_{l=1}^{N-n} \prod_{j=1}^{n}\left|z_{j}-\xi_{l}\right|^{2} \int d \Lambda a(\Lambda, \gamma) \Delta(\Lambda) \prod_{l=1}^{N-n} \sum_{c=1}^{M} \frac{e^{i \operatorname{Im} \xi_{l} \lambda_{c}}}{\prod_{s(\neq c)}\left(\lambda_{c}-\lambda_{s}\right)}
\end{aligned}
$$

where

$$
\begin{gathered}
a(\Lambda, \gamma)=e^{-i \sum_{c=1}^{M} \tilde{\gamma}_{c} \lambda_{c}} \prod_{j=1}^{n} \sum_{\kappa=1}^{M} \frac{e^{i \operatorname{Im} z_{j} \lambda_{\kappa}}}{\prod_{s(\neq \kappa)}\left(\lambda_{\kappa}-\lambda_{s}\right)}=\sum_{\kappa_{1}=1}^{M} \ldots \sum_{\kappa_{n}=1}^{M} A_{\kappa}(\Lambda, \gamma) \\
A_{\kappa}(\Lambda, \gamma)=\frac{e^{-i \sum_{c=1}^{M} \tilde{\gamma}_{c} \lambda_{c}}}{\prod_{j=1}^{n} \prod_{s\left(\neq \kappa_{j}\right)}^{M}\left(\lambda_{\kappa_{j}}-\lambda_{s}\right)} .
\end{gathered}
$$

In Eq. (B.2) we denoted $\kappa=\left(\kappa_{1}, \ldots, \kappa_{n}\right)$ and $\tilde{\gamma}_{c}=\gamma_{c}-\sum_{j=1}^{n} \delta_{c, \kappa_{j}} \operatorname{Im} z_{j}$, where $\delta_{c, \kappa_{j}}=1$ if $c=\kappa_{j}$ and zero otherwise. This allows us to write:

$$
R_{n}(\{z\}) \propto \frac{e^{-\frac{N-n}{2} \sum_{j=1}^{n} \operatorname{Re} z_{j}^{2}}|\Delta(\{z\})|^{2}}{\operatorname{det} \gamma^{n}} \sum_{\kappa_{1}=1}^{M} \ldots \sum_{\kappa_{n}=1}^{M} F_{\kappa}(\gamma,\{z\}),
$$

where

$$
\begin{gathered}
F_{\kappa}(\gamma,\{z\})=B(\gamma) \int d^{2}\{\xi\} e^{-\frac{N-n}{2} \sum_{l=1}^{N-n} \operatorname{Re} \xi_{l}^{2}}|\Delta(\{\xi\})|^{2} \prod_{l=1}^{N-n} \prod_{j=1}^{n}\left|z_{j}-\xi_{l}\right|^{2} \\
\times \int d \Lambda A_{\kappa}(\Lambda, \gamma) \Delta(\Lambda) \prod_{l=1}^{N-n} \sum_{c=1}^{M} \frac{e^{i \operatorname{Im} \xi_{l} \lambda_{c}}}{\prod_{s(\neq c)}\left(\lambda_{c}-\lambda_{s}\right)}
\end{gathered}
$$

and we denoted

$$
B(\gamma)=\frac{e^{-\frac{N-n}{2} \sum_{c=1}^{M} \gamma_{c}^{2}}}{\Delta(\gamma) \operatorname{det} \gamma^{N-n-M}} .
$$

Now we introduce the differential operators $\hat{D}_{\kappa}=\prod_{j=1}^{n} \prod_{s\left(\neq \kappa_{j}\right)}^{M}\left(\frac{\partial}{\partial \gamma_{k_{j}}}-\frac{\partial}{\partial \gamma_{s}}\right)$ and consider its action on the ratio $F_{\kappa}(\gamma) / B(\gamma)$ from Eq. (B.4). On one hand, we notice that:

$$
\hat{D}_{\kappa} A_{\kappa}(\lambda, \gamma)=(-i)^{n(M-1)} e^{-i \sum_{c=1}^{M} \tilde{\gamma}_{c} \lambda_{c}} .
$$


and, as a result the action of $\hat{D}_{\kappa}$ on $F_{\kappa}(\gamma) / B(\gamma)$ brings the $\xi$-integrand exactly to the form that allows us to rewrite it as an average of a product of determinants (cf.Eq.(221)). The averaging goes over the density of joint probability of eigenvalues $\xi_{1}, \xi_{2}, \ldots, \xi_{N-n}$ of a non-Hermitian matrix $J_{N-n}(\tilde{\gamma})=H_{N-n}+i \tilde{\Gamma}$, with nonzero eigenvalues of $\tilde{\Gamma}$ being $\tilde{\gamma_{1}}, \ldots, \tilde{\gamma_{M}}$ :

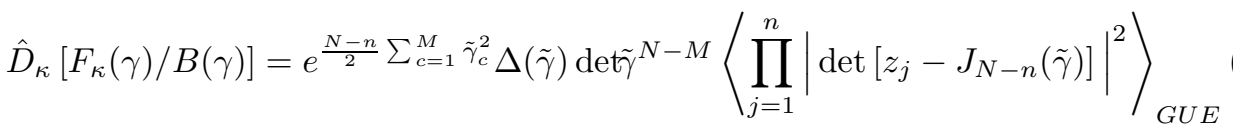

On the other hand, to ensure existence of a well-defined limit $N \rightarrow \infty$ of the correlation functions $R_{n}(\{z\})$ in Eq.(B.3) the quantities $F_{\kappa}(\gamma)$ must in that limit behave regularly as functions of $\gamma_{c}$, i.e. $\left.F_{\kappa}(\gamma)\right|_{N \gg 1} \sim C_{N} F_{\kappa}^{(s)}(\gamma)$ where $C_{N}$ does not depend on $\gamma$ and $F_{\kappa}^{(s)}(\gamma)$ has a finite limit when $N \rightarrow \infty$. Because of this property in the limit $N \gg n$ the action of $\hat{D}_{\kappa}$ on

$$
F_{\kappa}(\gamma) / B(\gamma) \equiv F_{\kappa}(\gamma) \Delta(\gamma) \operatorname{det} \gamma^{N-n-M} e^{\frac{N-n}{2} \sum_{c=1}^{M} \gamma_{c}^{2}}
$$

to the leading order amounts to:

$$
\hat{D}_{\kappa}\left[F_{\kappa}(\gamma) / B(\gamma)\right] \approx F_{\kappa}(\gamma) \Delta(\gamma) \hat{D}_{\kappa}\left[\operatorname{det} \gamma^{N-n-M} e^{\frac{N-n}{2} \sum_{c=1}^{M} \gamma_{c}^{2}}\right]
$$

Indeed, each $\gamma$-differentiation of the terms in the square brackets in Eq. (B.6) brings a factor of the order of $N$, which is much larger compared to the result of differentiating the factor $F_{\kappa}(\gamma) \Delta(\gamma)$. Performing the remaining differentiations explicitly and considering $N-n \approx N-n-M \approx N$, we find:

$$
\begin{aligned}
\hat{D}_{\kappa}\left[F_{\kappa}(\gamma) / B(\gamma)\right] & \left.\right|_{N \gg n, M} \approx(2 N)^{n(M-1)} F_{\kappa}(\gamma) \Delta(\gamma) \\
& \times \operatorname{det} \gamma^{N-n-M} e^{\frac{N-n}{2} \sum_{c=1}^{M} \gamma_{c}^{2}} \prod_{j=1}^{n} \prod_{s\left(\neq \kappa_{j}\right)}^{M}\left(g_{\kappa_{j}}-g_{s}\right)
\end{aligned}
$$

where we introduced the notation $g_{c}=\frac{1}{2}\left(\gamma_{c}+\gamma_{c}^{-1}\right)$.

Comparing this expression with Eq. (B.5) yields

$F_{\kappa}(\gamma,\{z\}) \propto \frac{C_{\tilde{\gamma}}(\{z\})}{\prod_{j=1}^{n} \prod_{s\left(\neq \kappa_{j}\right)}^{M}\left(g_{\kappa_{j}}-g_{s}\right)} \frac{\Delta(\tilde{\gamma})}{\Delta(\gamma)}\left[\operatorname{det} \frac{\tilde{\gamma}}{\gamma}\right]^{N-n-M} e^{-\frac{N-n}{2} \sum_{c=1}^{M}\left(\gamma_{c}^{2}-\tilde{\gamma}_{c}^{2}\right)}$

where we denoted:

$$
C_{\tilde{\gamma}}(\{z\})=\left\langle\prod_{j=1}^{n}\left|\operatorname{det}\left[z_{j}-J_{N-n}(\tilde{\gamma})\right]\right|^{2}\right\rangle_{G U E} .
$$

In the limit when $N \rightarrow \infty$ and $M$ finite, almost all eigenvalues of $J$ have imaginary part of the order $\frac{1}{N} \ll \gamma_{c}$ [90. Rescaling the imaginary parts $y_{j}=N \operatorname{Im} z_{j}$ one finds that

$$
\frac{\Delta(\tilde{\gamma})}{\Delta(\gamma)}\left[\operatorname{det} \frac{\tilde{\gamma}}{\gamma}\right]^{N-n-M} e^{-\frac{N-n}{2} \sum_{c=1}^{M}\left(\gamma_{c}^{2}-\tilde{\gamma}_{c}^{2}\right)}=e^{-2 \sum_{j=1}^{n} y_{j} g_{\kappa_{j}}}
$$

in the limit $n, M \ll N \rightarrow \infty$. As will be clear later on, in the same limit one can substitute $\gamma$ for $\tilde{\gamma}$ in the GUE averaged product of determinants. Combining all the factors we arrive at Eq.(32).

[1] Guhr T, Muller-Groeling A and HA Weidenmuller 1998 Phys Rep 299190

[2] Berry M 1985 Proc.R.Soc.London Ser.A 400229 
[3] Bogomolny E and Keating J 1996 Phys.Rev.Lett 771472

[4] Muzykantsky BA and Khmelnitsky DE 1995 JETP Lett. 6276

[5] Andreev A, Agam O, Altshuler BL and Simons B 1996 Phys.Rev.Lett. 763947

[6] Verbaarschot JJM, Wettig T 2000 Annu. Rev. Nucl Part.Sci. 50343

[7] Slevin K and Nagao T 1994 Phys.Rev.B 502380

[8] Beenakker CWJ 1997 Rev.Mod.Phys. 69731

[9] Verbaarschot JJM, Weidenmüller HA and Zirnbauer MR 1985 Phys.Rep. 129367

[10] Lewenkopf CH and Weidenmüller HA 1991 Ann.Phys. NY212 53

[11] Fyodorov YV and Sommers H-J 1997 J.Math. Phys. 381918

[12] Dittes FM 2000 Phys. Rep. 339216 ; Pichugin K, Schanz H and Seba P 2001 Phys.Rev.E 64 art.No 056227

[13] Ullah N 1969 J.Math.Phys. 10 2099; Sokolov VV and Zelevinsky VG 1988 Phys.Lett. 202B 10; and 1989 Nucl.Phys.A 504 562; Stöckmann H-J and Seba P 1998 J.Phys.A:Math.Gen. 313439

[14] Haake F, Izrailev F, Lehmann N, Saher D, and Sommers H-J 1992 Z.Phys.B 88 359; Lehmann N, Saher D, Sokolov VV, and Sommers HJ 1995 Nucl.Phys.A 582 223; Müller M, Dittes F-M, Iskra W, and Rotter I (1995) Phys.Rev.E 525961

[15] Fyodorov YV and Sommers HJ 1996 Pis'ma ZhETF 63970 [Engl.transl. 1996 JETP Letters 63 1026]

[16] Gorin T, Dittes F-M, Müller M, Rotter I and Seligman TH 1997 Phys.Rev.E 562481

[17] John W, Milek B, Schanz H, and Seba P 1991 Phys.Rev.Lett. 67 1949; Drozdz S, Trellakis A, and Wambach J 1996 Phys.Rev.Lett. 76 4891; Hackenbroich G and Nöckel G Europh.Lett. 39371 (1997)

[18] Main J and Wunner G 1994 J.Phys.B: At.Mol. 272835

[19] Blümel R 1996 Phys.Rev.E 545420

[20] Mandelshtam VA and Taylor HS 1997a J.Chem.Soc.Faraday.Trans. 93847 and 1997b Phys.Rev.Lett. $\mathbf{7 8} 3274$

Kirmse B, Abel B, Schwarzer D, et al. 2000 J. Phys. Chem. A 10410398

[21] Grebenshchikov SY 2000 J Phys Chem A 10410409

[22] Kottos T and Smilansky U 2000 Phys.Rev.Lett. 85 968; see also Kottos T and Smilansky U 2003 J.Phys.A: v.36, 3501

[23] Glück M, Kolovski AR and Korsch HJ Phys.Rep 366 (2002) 103

[24] Smilansky U 1991 in Chaos and Quantum Physics. Proceedings of the Les-Houches Summer School. Session LII, ed. by Giannoni MJ et.al (North Holland, Amsterdam) p.372

[25] Mahaux C and Weidenmüller HA 1969 Shell Model Approach in Nuclear Reactions (North Holland, Amsterdam)

[26] Savin D.V., Sokolov V.V., Sommers H.-J. 2003 Phys.Rev.E 67, 026215

[27] Arov D Z 1979 Sib.Math.Journ. 20149 and Helton JW 1974 J.Funct.Anal. 1615

[28] Livšic MS Operators, Oscillations, Waves: Open Systems, Amer.Math.Soc.Trans. 34 (Am.Math.Soc.,Providence,RI,1973)

[29] Efetov KB Supersymmetry in Disorder and Chaos (Cambridge University Press,1996)

[30] Gorin T and Seligman TH 2002 Phys.Rev.E 65 026214; Fyodorov Y.V., Alhassid Y. 1998 Phys.Rev.A 583375

[31] Albeverio S, Haake F, Kurasov P, Kus M and Seba P 1996 J.Math.Phys. 37 4888; Ishio H 2000 Phys.Rev.E 62 R3035

[32] Smilansky U 1999 in: Supersymmetry and Trace Formulae: Chaos and Disorder p.173, ed. by Lerner I V et al. (Kluwer, NY)

[33] Haake F. 1999 Quantum Signatures of Chaos (Springer, 2nd ed.)

[34] Borgonovi F, Guarneri I and Shepelyansky D L 1991 Phys.Rev. A 434517 and Borgonovi F, Guarneri I 1993 Phys.Rev. E 48 R2347

[35] Mello P A and Pichard J L 1991 J.de Phys. I 1493

[36] Życzkowski K and Sommers H-J 2000 J. Phys. A: Math. Gen. 332045

[37] A.Horn 1954 Proc. Am. Math. Soc 5 4; H. Weyl 1949 Proc.Nat.Acad.Sci. USA 35408

[38] Stephanov MA 1996 Phys.Rev.Lett. 764472

[39] Halasz MA, Jackson AD, Verbaarschot JJM 1997 Phys Rev D 56 5140; Halasz MA, Osborn JC, Verbaarschot JJM 1997 Phys Rev D 56 7059;

Halasz MA, Osborn JC, Stephanov MA, Verbaarschot JJM 2000 Phys Rev D 61076005 ; Kogut JB, Stephanov MA, Toublan D, et al. 2000 Nucl. Phys.B 582 477;

Toublan D and Verbaarschot JJM 2001 Int. J. Mod. Phys. B 151404

[40] Markum H, Pullirsch R, Wettig T 1999 Phys Rev Lett 83484 
[41] Janik RA, Nowak MA, Papp G, Zahed I 1997 Nucl.Phys.B 501603 and 1998 Nucl.Phys.A 642 191; Feinberg J and Zee A 1997a Nucl.Phys.B $\mathbf{5 0 4} 579$ and 1997b Nucl.Phys.B 501643

[42] Akemann G 2001 Phys.Rev. D 64114021

[43] Akemann G 2002 Phys.Rev.Lett. 89 Art.No. 072002 and arXiv:hep-th/0204246

[44] Akemann G 2002 Phys. Lett. B 547 (2002), 100

[45] Efetov KB 1997a Phys.Rev.Lett. 79491 and 1997b Phys.Rev.B 569630

[46] Izyumov AV, Simons BD 1999a Phys. Rev. Lett. 834373 and 1999b Europh. Lett. 45 290; Marchetti FM and Simons BD 2001 J. Phys. A 34 (2001) 10805

[47] Fyodorov YV, Khoruzhenko B and Sommers H-J 1997a Physics Letters A 22646

[48] J.Feinberg, R.Scalettar, and A.Zee 2001 J.Math.Phys. 42 5718; P. Wiegmann and A.Zabrodin 2003 J.Phys. A: Math.Phys. 363411 , A.Zabrodin 2002, arXiv:cond-mat/0210331

[49] M.L.Mehta 1990 Random Matrices (Academic Press Inc., N.Y., 2nd ed. )

[50] Fyodorov YV, Khoruzhenko B and Sommers H-J 1997 Phys.Rev.Lett. 79557 and 1998 Ann.Inst.Henri Poincare: Phys. Theor. 68449

[51] Fyodorov YV and Khoruzhenko B 1999 Phys.Rev.Lett. 8365

[52] Sommers H-J, Fyodorov YV and Titov M 1999 J.Phys. A: Math.Gen. 32 L77

[53] Chalker J T and Mehlig B 1998 Phys.Rev.Lett. 81 3367; Mehlig B J and Chalker J T 2000 J.Math.Phys. 41 3233; Chalker J T and Wang Z J 1997 Phys.Rev.Lett. 791797

[54] Schomerus H, Frahm K M, Patra M and Beenakker C W J 2000 Physica A 278 469; Frahm K, Schomerus H, Patra M and Beenakker C W J 2000 Europh. Lett. 49 48; Patra M, Schomerus H and Beenakker C W J 2000 Phys.Rev.A 6123810

[55] Janik R A, Noerenberg W, Nowak M A, Papp G and Zahed I 1999 Phys.Rev.E 602699

[56] Mehlig B and Santer M 2001 Phys.Rev.E 63020105

[57] Fyodorov Y V and Mehlig B 2002 Phys.Rev.E 66045202 cond-mat/0207166

[58] Hackenbroich G, Viviescas C and Haake F 2002 Phys. Rev. Lett.89 art. no. 083902

[59] Viviescas C and Hackenbroich G 2003 Phys. Rev. A67, 013805

[60] Petermann K 1979 IEEE J.Quantum Electron 15 566; Kaus H A and Kawakami S 1985 IEEE J.Quantum Electron 21 63; A.E. Siegman 1989 Phys.Rev.A 391253 and ibid 391264

[61] Fyodorov YV, Titov M and Sommers H-J 1998 Phys.Rev.E 581195

[62] Fyodorov Y V 2001 in Disordered and Complex Systems edited by P.Sollich et al. AIP Conference Proceedings $\mathbf{5 5 3} 191$ (Melville NY) arXiv:nlin.CD/0002034

[63] Fyodorov Y V and Sommers H-J 2000 JETP Letters 72422

[64] Fyodorov Y V 1998 From Dyson to Ginibre eigenvalue statistics Proceedings of Warwick Seminar on Random Matrices, available at http://www.maths.warwick.ac.uk/ olegz/ws1998/index.html

[65] Edelman A 1997 J. Multivariate Anal. 60 203; Edelman A, Kostlan E and Shub M J.Am.Math.Soc. 7247 (1994)

[66] Di Francesco F, Gaudin M, Itzykson C, and Lesage F 1994 Int.J.Mod.Phys.A 94257

[67] Forrester PJ and Jancovici B 1997 Int.J.Mod.Phys.A 11941

[68] Szegö G 1975 Orthogonal polynomials 4th ed. (AMS, Providence) p. 380

[69] Ginibre J 1965 J. Math. Phys. 6440

[70] V. Girko 1986 Theor. Prob. Appl. 30677 H.-J.Sommers, A.Crisanti, H.Sompolinsky, and Y.Stein 1989 Phys.Rev.Lett. 601895

[71] Z.D.Bai 1997 Ann.Prob. 25494

[72] Kolesnikov AV and Efetov KB 2000 Phys.Rev.Lett. 845600 and 1999 Waves in Random Media 971

[73] Hastings MB 2000 Nucl.Phys.B 572535

[74] Nishigaki SM and Kamenev A 2002 J.Phys.A: Math.Gen. 354571

[75] Kanzieper E J Phys.A: Math.Gen. 2002 35, 6631

[76] Garcia-Garcia AM, Nishigaki SM, Verbaarschot JJM Phys. Rev. E 200266 art. No. 016132

[77] D.Savin, Y.V.Fyodorov, H.-J.Sommers 2001 Phys.Rev.E 63 035202(R)

[78] C.Itzykson and J.B.Zuber 1980 J.Math.Phys 21 411; Harish-Chandra Am.J.Math. $1957 \mathbf{7 9} 87$

[79] N.Lehmann and H.-J.Sommers 1991 Phys.Rev.Lett. 67941

[80] Grobe R, Haake F and Sommers H-J 1988 Phys.Rev.Lett. 611899

[81] Y.V.Fyodorov and B.A.Khoruzhenko, unpublished; In fact, related expressions, in a different form, appeared in a recent preprint: A. Orlov, arXiv:math-ph/0210012

[82] Haake F, Kuś M, Sommers H-J and Życzkowski K 1996 J.Phys.A:Math.Gen. 293641

[83] J.P.Keating and N.C.Snaith 2000 Comm.Math.Phys. 214 (2000), 57

[84] Savin DV and Sokolov VV 1997 Phys.Rev.E 56 R4911 (1997) 
[85] Izrailev F, Saher D and Sokolov V V 1994 Phys.Rev.E 49130

[86] E. Brezin and S. Hikami 2000 Commun. Math. Phys. 214 111; Fyodorov YV 2002 Nucl. Phys. B [PM] 621 643; Fyodorov YV and E. Strahov 2002 Nucl. Phys. B [PM] 630453

[87] Fyodorov YV and E. Strahov 2003 J.Phys.A:Math.Gen. 36, 3203 and E. Strahov; Y.V. Fyodorov 2002 arXiv:math-phys/0210010

[88] Kogan E, Mello P A and Liqun H 2000 Phys. Rev. E 61 R17; Beenakker C W J and Brouwer P W 2001 Physica E 9463

[89] T.Kottos and M.Weiss 2002 Phys.Rev.Lett. 89 056401; Titov M, Fyodorov YV 2000 Phys.Rev.B 61 R2444; M.Terraneo, I.Guarneri 2000 Eur.Phys.J. B. 18, 303; M.Patra 2002 arXiv:cond-mat/0206020

[90] Precisely speaking, the fraction $m$ of resonances having widths $\operatorname{Im} z \sim N^{-1}$ is known to satisfy $1-M / N \leq m \leq 1$, see 11 pp.1949-1950

[91] Fyodorov YV Nucl.Phys.B 621[PM] (2002), 643 (see Appendix A of that paper).

[92] Janik RA and Nowak MA 2003 J Phys.A:Math.Gen. 36, 3629 